\title{
Quantum Spin Dynamics (QSD) : VII. Symplectic Structures and Continuum Lattice Formulations of Gauge Field Theories
}

\author{
T. Thiemann* \\ MPI f. Gravitationsphysik, Albert-Einstein-Institut, \\ Am Mühlenberg 1, 14476 Golm near Potsdam, Germany \\ Preprint AEI-2000-026
}

\begin{abstract}
Interesting non-linear functions on the phase spaces of classical field theories can never be quantized immediately because the basic fields of the theory become operator valued distributions. Therefore, one is usually forced to find a classical substitute for such a function depending on a regulator which is expressed in terms of smeared quantities and which can be quantized in a well-defined way. Namely, the smeared functions define a new symplectic manifold of their own which is easy to quantize. Finally one must remove the regulator and establish that the final operator, if it exists, has the correct classical limit.

In this paper we investigate these steps for diffeomorphism invariant quantum field theories of connections. We introduce a generalized projective family of symplectic manifolds, coordinatized by the smeared fields, which is labelled by a pair consisting of a graph and another graph dual to it. We show that there exists a generalized projective sequence of symplectic manifolds whose limit agrees with the symplectic manifold that one started from.

This family of symplectic manifolds is easy to quantize and we illustrate the programme outlined above by applying it to the Gauss constraint. The framework developed here is the classical cornerstone on which the semi-classical analysis developed in a new series of papers called "Gauge Theory Coherent States" is based.

This article also complements, as a side result, earlier work by Ashtekar, Corichi and Zapata who observed that certain operators are non-commuting on certain states although the Poisson brackets between the classical functions that these authors based the quantization on, vanish. We show that there are other functions on the classical phase space which give rise to the same operators but whose Poisson algebra precisely mirrors the quantum commutator algebra.
\end{abstract}

\section{Introduction}

Quantum General Relativity (QGR) has matured over the past decade to a mathematically welldefined theory of quantum gravity. In contrast to string theory, by definition, GQR is a manifestly background independent, diffeomorphism invariant and non-perturbative theory. The obvious advantage is that one will never have to postulate the existence of a non-perturbative

*thiemann@aei-potsdam.mpg.de 
extension of the theory, which in string theory has been called the still unknown M(ystery)Theory.

The disadvantage of a non-perturbative and background independent formulation is, of course, that one is faced with new and interesting mathematical problems so that one cannot just go ahead and "start calculating scattering amplitudes": As there is no background around which one could perturb, rather the full metric is fluctuating, one is not doing quantum field theory on a spacetime but only on a differential manifold. Once there is no (Minkowski) metric at our disposal, one loses familiar notions such as causality structure, locality, Poincaré group and so forth, in other words, the theory is not a theory to which the Wightman axioms apply. Therefore, one must build an entirely new mathematical apparatus to treat the resulting quantum field theory which is drastically different from the Fock space picture to which particle physicists are used to.

As a consequence, the mathematical formulation of the theory was the main focus of research in the field over the past decade. The main achievements to date are the following (more or less in chronological order) :

\section{i) Kinematical Framework}

The starting point was the introduction of new field variables [1] for the gravitational field which are better suited to a background independent formulation of the quantum theory than the ones employed until that time. In its original version these variables were complex valued, however, currently their real valued version, considered first in [2] for classical Euclidean gravity and later in [3] for classical Lorentzian gravity, is preferred because to date it seems that it is only with these variables that one can rigorously define the dynamics of Euclidean or Lorentzian quantum gravity [4].

These variables are coordinates for the infinite dimensional phase space of an $S U(2)$ gauge theory subject to further constraints besides the Gauss law, that is, a connection and a canonically conjugate electric field. As such, it is very natural to introduce smeared functions of these variables, specifically Wilson loop and electric flux functions. (Notice that one does not need a metric to define these functions, that is, they are background independent). This had been done for ordinary gauge fields already before in [5] and was then reconsidered for gravity (see e.g. [6]).

The next step was the choice of a representation of the canonical commutation relations between the electric and magnetic degrees of freedom. This involves the choice of a suitable space of distributional connections [7] and a faithful measure thereon [8] which, as one can show [9], is $\sigma$-additive. The proof that the resulting Hilbert space indeed solves the adjointness relations induced by the reality structure of the classical theory as well as the canonical commutation relations induced by the symplectic structure of the classical theory can be found in [10]. Independently, a second representation, called the loop representation, of the canonical commutation relations had been advocated (see e.g. 11] and especially 12 and references therein) but both representations were shown to be unitarily equivalent in [13] (see also 14] for a different method of proof).

This is then the first major achievement : The theory is based on a rigorously defined kinematical framework.

\section{ii) Geometrical Operators}

The second major achievement concerns the spectra of positive semi-definite, self-adjoint geometrical operators measuring lengths [15], areas [16, 17] and volumes [16, 18, 19, 20, 11] of curves, surfaces and regions in spacetime. These spectra are pure point (discete) and imply a discrete Planck scale structure. It should be pointed out that the discreteness is, in contrast to other approaches to quantum gravity, not put in by hand but it is a prediction! 
iii) Regularization- and Renormalization Techniques

The third major achievement is that there is a new regularization and renormalization technique [21, 22] for diffeomorphism covariant, density-one-valued operators at our disposal which was successfully tested in model theories [23]. This technique can be applied, in particular, to the standard model coupled to gravity [24, 25] and to the Poincaré generators at spatial infinity [26]. In particular, it works for Lorentzian gravity while all earlier proposals could at best work in the Euclidean context only (see, e.g. [12] and references therein). The algebra of important operators of the resulting quantum field theories was shown to be consistent [27]. Most surprisingly, these operators are $U V$ and IR finite! Notice that this result, at least as far as these operators are concerned, is stronger than the believed but unproved finiteness of scattering amplitudes order by order in perturbation theory of the five critical string theories, figuratively speaking, we claim that our perturbation series converges. The absence of the divergences that usually plague interacting quantum fields propagating on a Minkowski background can be understood intuitively from the diffeomorphism invariance of the theory : "short and long distances are gauge equivalent". We will elaborate more on this point in future publications. The classical limit of the above mentioned operators will be studied in our companion paper [28].

iv) Spin Foam Models

After the construction of the densely defined Hamiltonian constraint operator of [21, 22], a formal, Euclidean functional integral was constructed in [29] and gave rise to the socalled spin foam models (a spin foam is a history of a graph with faces as the history of edges) [30]. Spin foam models are in close connection with causal spin-network evolutions [31], state sum models [32 and topological quantum field theory, in particular BF theory [33]. To date most results are at a formal level and for the Euclidean version of the theory only but the programme is exciting since it may restore manifest four-dimensional diffeomorphism invariance which in the Hamiltonian formulation is somewhat hidden.

v) Finally, the fifth major achievement is the existence of a rigorous and satisfactory framework [34, 35, 36, 37, 38, 39, 40] for the quantum statistical description of black holes which reproduces the Bekenstein-Hawking Entropy-Area relation and applies, in particular, to physical Schwarzschild black holes while stringy black holes so far are under control only for extremal charged black holes.

Summarizing, the work of the past decade has now culminated in a promising starting point for a quantum theory of the gravitational field plus matter and the stage is set to address physical questions. In particular, one would like to make contact with the language that particle physicists are more familiar with, that is, perturbation theory. In other words, one should be able to define something like gravitons and photons propagating on a fluctuating quantum spacetime. By this we mean the following :

Suppose we want to study the semi-classical limit of our quantum gravity theory, that is, a limit in which the gravitational field behaves almost classical. This does not mean that we want to treat gravity as a background field [41], rather we take all the quantum fluctuations into account but try to find a state with respect to which those fluctuations (around the Minkowski metric) are minimal. With respect to such a "background state" one can study relative excitations of the gravitational field (gravitons) or of matter fields (such as photons).

In order to do this we must first develop an appropriate semi-classical framework which we will do in 42, 43, 44. But even before doing this we must examine the following issue which seems not to have been sufficiently appreciated throughout the literature so far : Namely, the quantum theory is based on certain configuration and conjugate momentum variables respectively, specifically holonomy - and electric flux variables. We stress that we use here non-standard flux variables not previously considered in the literature. These non-local 
functions on the classical phase space are, in particular, used to regularize more complicated composite operators such as the geometrical operators mentioned above. It is already quite remarkable that one can remove the regulator without encountering any UV divergencies! However, in order to be convinced that this regulator-free operator really has the correct classical limit one has to check, for instance, that it has the correct expectation values with respect to semi-classical states. The question arises how such semi-classical states should look like. Now, since the final regulator-free operator is actually only densely defined (since it is usually unbounded) one has to employ states which are semi-classical and simultaneously belong to a dense subspace of the Hilbert space. The states which belong to the domain of definition of the operator are labelled by graphs $\gamma$. Given such a graph $\gamma$ one can define unambiguously holonomies along its edges as the basic configuration operators labelled by $\gamma$, however, the associated (conjugate) momentum operators are largely ambiguous in the sense that any choice of surfaces which are mutually disjoint and are intersected by precisely one edge of $\gamma$ gives rise to completely identical Poisson brackets between the canonical variables.

This then leads to the following problem : Suppose we define a semi-classical state by requiring that the expectation value of the basic holonomy and electric flux operators associated with $\gamma$ take certain values and satisfy a minimal uncertainty property. Given a point in the classical phase space those values should be the values of the holonomy and flux functions evaluated at that point. However, this makes sense only when we specify the surfaces with respect to which we calculate the flux.

We are thus led to invent a new kind of generalized projective family labelled not only by graphs but also by so-called "dual" faces. In particular, we wish to do this already at the classical level by introducing a new kind of generalized projective family of symplectic manifolds. The idea behind all of this is that these symplectic manifolds enable us to discuss in a clean way the quantization procedure and its inverse, the process of taking the classical limit :

\section{Classical Regularization}

Suppose we are given a function on the classical phase space $(M, \Omega)$, usually a function $F(m)$ of the connection and the electric field, $m=(A, E)$. Here $M$ denotes the set of connections and electric fields respectively (a differentiable manifold modelled on a Banach space, see below) and $\Omega$ is a strong symplectic structure on $M$. As we cannot define $\hat{A}, \hat{E}$ on our Hilbert space directly as operators, we must first find a substitute $F_{\gamma}(m)$ for $F$ which can be written entirely in terms of holonomy and flux variables associated with $\gamma$. These variables coordinatize a symplectic manifold $\left(M_{\gamma}, \Omega_{\gamma}\right)$. We will say that $F_{\gamma}(m)$ is a substitute for $F(m)$ provided that 1) $F_{\gamma}$ converges to $F$ pointwise on $M$ as $\gamma \rightarrow \infty$ (the graph becomes infinitely fine, we will specify this limit below) and 2) that the Hamiltonian vector field of $F_{\gamma}$ with respect to $\Omega_{\gamma}$ converges pointwise on $M$ to that of $F$ with respect to $\Omega$.

\section{Regularized Operators}

The classical phase spaces $\left(M_{\gamma}, \Omega_{\gamma}\right)$ turn out to be (in)finite direct products of (copies of) cotangent spaces over the gauge group $G$ equipped with a non-standard symplectic structure and allow for a bona fide quantization by usual geometrical quantization techniques. By substituting classical variables for operators defined on a subspace $\mathcal{H}_{\gamma}$ of the Hilbert space and Poisson brackets with respect to $\Omega_{\gamma}$ by commutators we obtain an

operator $\hat{F}_{\gamma}$ unambiguously defined on $\mathcal{H}_{\gamma}$ up to factor ordering ambiguities. Thus, the phase spaces $M_{\gamma}$ are much better suited for the quantization of interesting functions $F$ on $M$ as they are automatically finite and we have always control that the quantization has the correct classical limit on $M_{\gamma}$. In other words, quantization and regularization can be neatly separated as individual processes. 


\section{Unregularized Operator}

It turns out that for a large class of functions $F$ including the ones of physical interest the family of operators $\hat{F}_{\gamma}$ so obtained provides an operator $\hat{F}$ consistently defined on a dense subspace of the whole Hilbert space in the sense that its restriction to $\mathcal{H}_{\gamma}$ coincides with $\hat{F}_{\gamma}$. This will be our candidate for a well-defined continuum operator.

\section{Classical Limit}

In order to study the classical limit of $\hat{F}$ we introduce a generalized projective family of semi-classical states $\psi_{\gamma, m}^{t} \in \mathcal{H}_{\gamma}$ labelled by the graph $\gamma$, a point in $m \in M$ and a classicality parameter $t \propto \hbar$. We say that $\hat{F}_{\gamma}$ is a quantization of $F_{\gamma}(m)$ provided that $\lim _{t \rightarrow 0}<\psi_{\gamma, m}^{t}, \hat{F}_{\gamma} \psi_{\gamma, m}^{t}>=F_{\gamma}(m)$ for each $m \in M$ and that $\hat{F}$ is a quantization of $F$ provided that $\lim _{t \rightarrow 0}\left[\lim _{\gamma \rightarrow \infty}<\psi_{\gamma, m}^{t}, \hat{F}_{\gamma} \psi_{\gamma, m}^{t}>\right]=F(m)$ for each $m \in M$.

Theses four steps provide then a closed path of how to go from a classical phase space function to an operator and back. As we see, this procedure requires as a classical cornerstone the analysis of the phase spaces $\left(M_{\gamma}, \Omega_{\gamma}\right)$ which is the subject of the present paper. In particular, one must show that these symplectic manifolds contain a generalized projective sequence that can be identified with $(M, \Omega)$.

The outline of the paper is as follows :

In section two we recall a working collection of material from the kinematical framework of the theory.

In section three we derive from the symplectic manifold $(M, \Omega)$ for gauge theories with compact gauge groups (in any dimension and on any (globally hyperbolic) manifold) a generalized projective family of (in)finite dimensional symplectic manifolds $\left(M_{\gamma}, \Omega_{\gamma}\right)$ labelled by graphs $\gamma$ embedded in that manifold. We show that the generalized projective limit symplectic manifold of a certain generalized projective sequence agrees with the standard symplectic manifolds $(M, \Omega)$ for gauge theories (weighted Sobolev spaces). The purpose of doing this is that the generalized family of symplectic manifolds is much better suited to quantization than the standard gauge theory phase space as outlined above.

In section four we propose a substitute $G_{\gamma}$ for an important function $G$ on the phase space of any gauge theory, namely the Gauss constraint and show that $G_{\gamma}$ converges to $G$ pointwise on $M$ in the generalized projective limit.

In section five we derive the quantization of $\left(M_{\gamma}, \Omega_{\gamma}\right)$ and $G_{\gamma}$. We show that $\hat{G}_{\gamma}$ is a consistently defined system of cylindrical projections of an operator $\hat{G}$ whose constraint algebra closes without anomalies. Finally we sketch the last step of the above programme applied to $\hat{G}$ concerning the classical limit. The proof that this step can be completed will be found in [42, 43, 44.

In section six we complement earlier results obtained by Ashtekar, Corichi and Zapata 45

These authors considered certain classical functions $F$ on $M$ and quantized them using $\Omega$ as a starting point. They obtained operators $\hat{F}$ this way which do not commute on certain states $f_{\gamma} \in \mathcal{H}_{\gamma}$ although the classical functions $F$ have vanishing Poisson brackets (with respect to $\Omega$ ) among each other. This seeming quantum "anomaly" was explained by pointing out that the connection and electric field of the theory are smeared with distributional rather than smooth test functions. If one uses a smearing with smooth functions then the "anomaly" vanishes, allowing the interpretation that the Poisson brackets of the unsmeared fields is non-vanishing, proportional to a distribution with support contained in a measurable subset of Lebesgue measure zero which is therefore detectable only when smearing with distributional smearing functions. This interpretation therefore removes the apparent contradiction. However, then one 
notices that this extended Poisson bracket does not close in an obvious way (the Jacobi identity is not obeyed in an obvious way). This was shown not to be an obstacle to quantization by recalling that it is not necessary to base the quantization on Poisson brackets but that one can instead base it on the Lie algebra of vector fields on $M$ which always obey the Jacobi identity and is always closed.

We show that the non-commutativity of these operators has a natural explanation from the point of view of the symplectic manifolds $\left(M_{\gamma}, \Omega_{\gamma}\right)$ :

1) We observe that we can find, for each of the above choices of $\gamma$, functions $F_{\gamma} \neq F$ which can be considered as functions on $M_{\gamma}$ as well. Furthermore, the functions $F_{\gamma}$ do have non-vanishing Poisson brackets among each other, both with respect to $\Omega$ and with respect to $\Omega_{\gamma}$ (actually, their brackets with respect to $\Omega_{\gamma}$ follow from those with resepct to $\Omega$ ).

2) The quantization of these new functions is such that $\hat{F}_{\gamma}$ and $\hat{F}$ agree on $\mathcal{H}_{\gamma}$.

3) The commutator algebra of the $\hat{F}$ on $\mathcal{H}_{\gamma}$ is precisely the one to be expected from the Poisson bracket structure of the $F_{\gamma}$.

In conclusion, the unexpected non-commutativity observed in [45] can be related to a quantization ambiguity. If we insist on a Poisson bracket - comutator correspondence principle, however, then one cannot accept the $F$ as classical limit of $\hat{F}$ but must instead consider the $F_{\gamma}$.

Finally, in an appendix we write the symplectic structure $\Omega_{\gamma}$ for $G=U(1), S U(2)$ in the language of differential forms which could be useful for future research.

\section{Preliminaries}

In this section we will recall the main ingredients of the mathematical formulation of diffeomorphism invariant quantum field theories of connections with local degrees of freedom in any dimension and for any compact gauge group. See [10] and references therein for more details.

Let $G$ be a compact gauge group, $\Sigma$ a $D$-dimensional manifold which admits a principal $G$-bundle with connection over $\Sigma$. Let us denote the pull-back to $\Sigma$ of the connection by local sections by $A_{a}^{i}$ where $a, b, c, . .=1, . ., D$ denote tensorial indices and $i, j, k, . .=1, . ., \operatorname{dim}(G)$ denote indices for the Lie algebra of $G$. We will denote the set of all smooth connections by $\mathcal{A}$ and endow it with a globally defined metric topology of the Sobolev kind

$$
d_{\rho}\left[A, A^{\prime}\right]:=\sqrt{-\frac{1}{N} \int_{\Sigma} d^{D} x \sqrt{\operatorname{det}(\rho)(x)} \operatorname{tr}\left(\left[A_{a}-A_{a}^{\prime}\right](x)\left[A_{b}-A_{b}^{\prime}\right](x)\right) \rho^{a b}(x)}
$$

where $\operatorname{tr}\left(\tau_{i} \tau_{j}\right)=-N \delta_{i j}$ is our choice of normalization for the generators of a Lie algebra $\operatorname{Lie}(G)$ of rank $N$ and our conventions are such that $\left[\tau_{i}, \tau_{j}\right]=2 f_{i j}{ }^{k} \tau_{k}$ define the structure constants of $\operatorname{Lie}(G)$. Here $\rho_{a b}$ is a fiducial metric on $\Sigma$ of everywhere Euclidean signature. In what follows we assume that either $D \neq 2$ ( for $D=2$, (2.1) depends only on the conformal structure of $\rho$ and cannot guarantee convergence for arbitrary fall-off conditions on the connections) or that $D=2$ and the fields $A$ are Lebesgue integrable.

Let $\Gamma_{0}^{\omega}$ be the set of all piecewise analytic, oriented graphs $\gamma$ embedded into $\Sigma$ and denote by $E(\gamma)$ and $V(\gamma)$ respectively its sets of oriented edges $e$ and vertices $v$ respectively. One can extend the framework to certain, tame piecewise smooth graphs 46, 47] but the description becomes more complicated and we refrain from doing this here. More important is the extension to infinite piecewise analytical graphs $\Gamma_{\sigma}^{\omega}$ about which much will be said in the first reference of 44]. For the purpose of this paper it will be sufficient to stick to $\Gamma_{0}^{\omega}$ which is sufficient, e.g. if $\Sigma$ is compact. All the properties that are derived here for $\Gamma_{0}^{\omega}$ readily extend to $\Gamma_{\sigma}^{\omega}$ as one can easily check.

We denote by $h_{e}(A)$ the holonomy of $A$ along $e$ and say that a function $f$ on $\mathcal{A}$ is cylindrical with respect to $\gamma$ if there exists a function $f_{\gamma}$ on $G^{|E(\gamma)|}$ such that $f=p_{\gamma}^{*} f_{\gamma}=f \circ p_{\gamma}$ where 
$p_{\gamma}(A)=\left\{h_{e}(A)\right\}_{e \in E(\gamma)}$. The set of such functions is denoted by $\Phi_{\gamma}$. Holonomies are invariant under reparameterizations of the edge and in this article we take edges always to be analytic diffeomorphisms between $[0,1]$ and a one-dimensional submanifold of $\Sigma$. Gauge transformations are functions $g: \Sigma \mapsto G ; x \mapsto g(x)$ and they act on holonomies as $h_{e} \mapsto g(e(0)) h_{e} g(e(1))^{-1}$.

A particularly useful set of cylindrical functions are the so-called spin-netwok functions 48, 49, 13. A spin-network function is labelled by a graph $\gamma$, a set of irreducible representations $\vec{\pi}=\left\{\pi_{e}\right\}_{e \in E(\gamma)}$ (choose from each equivalence class of equivalent representations once and for all a fixed representant), one for each edge of $\gamma$, and a set $\vec{c}=\left\{c_{v}\right\}_{v \in V(\gamma)}$ of contraction matrices, one for each vertex of $\gamma$, which contract the indices of the tensor product $\otimes_{e \in E(\gamma)} \pi_{e}\left(h_{e}\right)$ in such a way that the resulting function is gauge invariant. We denote spin-network functions as $T_{I}$ where $I=\{\gamma, \vec{\pi}, \vec{c}\}$ is a compound label. One can show that these functions are linearly independent.

The set of finite linear combinations of spin-network functions forms an Abelian * algebra $\mathcal{B}$ of functions on $\mathcal{A}$. By completing it with respect to the sup-norm topology it becomes an Abelian $\mathrm{C}^{*}$ algebra (here the compactness of $G$ is crucial). The spectrum $\overline{\mathcal{A}}$ of this algebra, that is, the set of all algebraic homomorphisms $\mathcal{B} \mapsto \mathbb{C}$ is called the quantum configuration space. This space is equipped with the Gel'fand topology, that is, the space of continuous functions $C^{0}(\overline{\mathcal{A}})$ on $\overline{\mathcal{A}}$ is given by the Gel'fand transforms of elements of $\mathcal{B}$. Recall that the Gel'fand transform is given by $\tilde{f}(\bar{A}):=\bar{A}(f) \forall \bar{A} \in \overline{\mathcal{A}}$. It is easy to see that $\overline{\mathcal{A}}$ with this topology is a compact Hausdorff space. Obviously, the elements of $\mathcal{A}$ are contained in $\overline{\mathcal{A}}$ and one can show that $\mathcal{A}$ is even dense [50]. Generic elements of $\overline{\mathcal{A}}$ are, however, distributional.

The idea is now to construct a Hilbert space consisting of square integrable functions on $\overline{\mathcal{A}}$ with respect to some measure $\mu$. Recall that one can define a measure on a locally compact Hausdorff space by prescribing a positive linear functional $\chi_{\mu}$ on the space of continuous functions thereon. The particular measure we choose is given by $\chi_{\mu_{0}}\left(\tilde{T}_{I}\right)=1$ if $I=\{\{p\}, \overrightarrow{0}, \overrightarrow{1}\}$ and $\chi_{\mu_{0}}\left(\tilde{T}_{I}\right)=0$ otherwise. Here $p$ is any point in $\Sigma, 0$ denotes the trivial representation and 1 the trivial contraction matrix. In other words, (Gel'fand transforms of) spin-network functions play the same role for $\mu_{0}$ as Wick-polynomials do for Gaussian measures and like those they form an orthonormal basis in the Hilbert space $\mathcal{H}:=L_{2}\left(\overline{\mathcal{A}}, d \mu_{0}\right)$ obtained by completing their finite linear span $\Phi$.

An equivalent definition of $\overline{\mathcal{A}}, \mu_{0}$ is as follows :

$\overline{\mathcal{A}}$ is in one to one correspondence, via the surjective map $H$ defined below, with the set $\overline{\mathcal{A}}^{\prime}:=\operatorname{Hom}(\mathcal{X}, G)$ of homomorphisms from the groupoid $\mathcal{X}$ of composable, holonomically independent, analytical paths into the gauge group. The correspondence is explicitly given by $\overline{\mathcal{A}} \ni \bar{A} \mapsto H_{\bar{A}} \in \operatorname{Hom}(\mathcal{X}, G)$ where $\mathcal{X} \ni e \mapsto H_{\bar{A}}(e):=\bar{A}\left(h_{e}\right)=\tilde{h}_{e}(\bar{A}) \in G$ and $\tilde{h}_{e}$ is the Gel'fand transform of the function $\mathcal{A} \ni A \mapsto h_{e}(A) \in G$. Consider now the restriction of $\mathcal{X}$ to $\mathcal{X}_{\gamma}$, the groupoid of composable edges of the graph $\gamma$. One can then show that the projective limit of the corresponding cylindrical sets $\overline{\mathcal{A}}_{\gamma}^{\prime}:=\operatorname{Hom}\left(\mathcal{X}_{\gamma}, G\right)$ coincides with $\overline{\mathcal{A}}^{\prime}$. Moreover, we have $\left\{\{H(e)\}_{e \in E(\gamma)} ; H \in \overline{\mathcal{A}}_{\gamma}^{\prime}\right\}=\left\{\left\{H_{\bar{A}}(e)\right\}_{e \in E(\gamma)} ; \bar{A} \in \overline{\mathcal{A}}\right\}=G^{|E(\gamma)|}$. Let now $f \in \mathcal{B}$ be a function cylindrical over $\gamma$ then

$$
\chi_{\mu_{0}}(\tilde{f})=\int_{\overline{\mathcal{A}}} d \mu_{0}(\bar{A}) \tilde{f}(\bar{A})=\int_{G^{|E(\gamma)|}} \otimes_{e \in E(\gamma)} d \mu_{H}\left(h_{e}\right) f_{\gamma}\left(\left\{h_{e}\right\}_{e \in E(\gamma)}\right)
$$

where $\mu_{H}$ is the Haar measure on $G$. As usual, $\mathcal{A}$ turns out to be contained in a measurable subset of $\overline{\mathcal{A}}$ which has measure zero with respect to $\mu_{0}$.

This concludes the definition of the kinematical Hilbert space $\mathcal{H}$, of the quantum configuration space $\overline{\mathcal{A}}$ and of the classical configuration space. What about the classical and quantum phase space? This question has actually so far not been analysed satisfactorily in the literature, partial results are scattered over a number of papers. We therefore begin the next section with this issue. 


\section{Symplectic Manifolds Labelled by Graphs}

\subsection{Standard Continuum Symplectic Structures}

Let us first recall the usual infinite dimensional symplectic geometry that underlies gauge theories.

Let $F_{i}^{a}$ be a Lie algebra valued vector density test field of weight one and let $f_{a}^{i}$ be a Lie algebra valued covector test field. Let, as before $A_{a}^{i}$ be a the pull-back of a connection to $\Sigma$ and consider a vector bundle of electric fields, that is, of Lie algebra valued vector densities of weight one whose bundle projection to $\Sigma$ we denote by $E_{i}^{a}$. We consider the smeared quantities

$$
F(A):=\int_{\Sigma} d^{D} x F_{i}^{a} A_{a}^{i} \text { and } E(f):=\int_{\Sigma} d^{D} x E_{i}^{a} f_{a}^{i}
$$

While both are diffeomorphism covarinat it is only the latter which is gauge covariant, one reason to consider the singular smearing functions discussed below. The choice of the space of pairs of test fields $(F, f) \in \mathcal{S}$ depends on the boundary conditions on the space of connections and electric fields which in turn depends on the topology of $\Sigma$ and will not be specified in what follows.

Consider the set $M$ of all pairs of smooth functions $(A, E)$ on $\Sigma$ such that (3.1) is well defined for any $(F, f) \in \mathcal{S}$. We wish to endow it with a manifold structure and a symplectic structure, that is, we wish to turn it into an infinite dimensional symplectic manifold.

We define a topology on $M$ through the metric :

$$
:=\sqrt{-\frac{1}{N} \int_{\Sigma} d^{D} x\left[\sqrt{\operatorname{det}(\rho)} \rho^{a b} \operatorname{tr}\left(\left[A_{a}-A_{a}^{\prime}\right]\left[A_{b}-A_{b}^{\prime}\right]\right)+\frac{\sigma_{a b} \operatorname{tr}\left(\left[E^{a}-E^{a \prime}\right]\left[E^{b}-E^{b^{\prime}}\right]\right)}{\sqrt{\operatorname{det}(\sigma)}}\right]}
$$

where $\rho_{a b}, \sigma_{a b}$ are again fiducial metrics on $\Sigma$ of everywhere Euclidean signature. Their fall-off behaviour has to be suited to the boundary conditions of the fields $A, E$ at spatial infinity. Notice that the metric (3.2) is gauge invariant (and thus globally defined) and diffeomorphism covariant and that $d_{\rho, \sigma}\left[(A, E),\left(A^{\prime}, E^{\prime}\right)\right]=d_{\rho}\left[A, A^{\prime}\right]+d_{\sigma}\left[E, E^{\prime}\right]$ (recall (2.1)).

Now, while the space of electric fields in Yang-Mills theory is a vector space, the space of connections is only an affine space. However, as we have also applications in general relativity with asymptotically Minkowskian boundary conditions in mind, also the space of electric fields will in general not be a vector space. Thus, in order to induce a norm from (3.2) we proceed as follows : Consider an atlas of $M$ consisting only of $M$ itself and choose a fiducial background connection and electric field $A^{(0)}, E^{(0)}$ (for instance $A^{(0)}=0$ ). We define the global chart

$$
\varphi: M \mapsto \mathcal{E} ;(A, E) \mapsto\left(A-A^{(0)}, E-E^{(0)}\right)
$$

of $M$ onto the vector space of pairs $\left(A-A^{(0)}, E-E^{(0)}\right)$. Obviously, $\varphi$ is a bijection. We topologize $\mathcal{E}$ in the norm

$$
\left\|\left(A-A^{(0)}, E-E^{(0)}\right)\right\|_{\rho \sigma}:=\sqrt{d_{\rho \sigma}\left[(A, E),\left(A^{(0)}, E^{(0)}\right)\right]}
$$

The norm (4.4) is of course no longer gauge and diffeomorphism covariant since the fields $A^{(0)}, E^{(0)}$ do not transform, they are backgrond fields. We need it, however, only in order to encode the fall-off behaviour of the fields which are independent of gauge - and diffeomorphism covariance.

Notice that the metric induced by this norm coincides with (3.2). In the terminology of weighted Sobolev spaces the completion of $\mathcal{E}$ in the norm (3.4) is called the Sobolev space 
$H_{0, \rho}^{2} \times H_{0, \sigma^{-1}}^{2}$, see e.g. [51]. We will call the completed space $\mathcal{E}$ again and its image under $\varphi^{-1}, M$ again (the dependence of $\varphi$ on $\left(A^{(0)}, E^{(0)}\right)$ will be suppressed). Thus, $\mathcal{E}$ is a normed, complete vector space, that is, a Banach space, in fact it is even a Hilbert space. Moreover, we have modelled $M$ on the Banach space $\mathcal{E}$, that is, $M$ acquires the structure of a (so far only topological) Banach manifold. However, since $M$ can be covered by a single chart and the identity map on $\mathcal{E}$ is certainly $C^{\infty}, M$ is actually a smooth manifold. The advantage of modelling $M$ on a Banach manifold is that one can take over almost all the pleasant properties from the finite dimensional case to the infinite dimensional one (in particular, the inverse function theorem).

Next we study differential geometry on $M$ with the standard techniques of calculus on infinite dimensional manifolds (see e.g. [52, 53]). We will not repeat all the technicalities of the definitions involved, the interested reader is referred to the literature quoted.

i) A function $f: M \mapsto \mathbb{C}$ on $M$ is said to be differentiable at $m$ if $g:=f \circ \varphi^{-1}: \mathcal{E} \mapsto \mathbb{C}$ is differentiable at $u=\varphi(m)$, that is, there exist bounded linear operators $D g_{u}, R g_{u}: \mathcal{E} \mapsto \mathbb{C}$ such that

$$
g(u+v)-g(u)=\left(D g_{u}\right) \cdot v+\left(R g_{u}\right) \cdot v \text { where } \lim _{\|v\| \rightarrow 0} \frac{\left|\left(R g_{u}\right) \cdot v\right|}{\|v\|}=0
$$

$D f_{m}:=D g_{u}$ is called the functional derivative of $f$ at $m$ (notice that we identify, as usual, the tangent space of $M$ at $m$ with $\mathcal{E}$ ). The definition extends in an obvious way to the case where $\mathbb{C}$ is replaced by another Banach manifold. The equivalence class of functions differentiable at $m$ is called the germ $G(m)$ at $m$. Here two functions are said to be equivalent provided they coincide in a neighbourhood containing $m$.

ii) In general, a tangent vector $v_{m}$ at $m \in M$ is an equivalence class of triples $\left(U, \varphi, v_{m}\right)$ where $(U, \varphi)$ is a chart of the atlas of $M$ containing $m$ and $v_{m} \in \mathcal{E}$. Two triples are said to be equivalent provided that $v_{m}^{\prime}=D\left(\varphi^{\prime} \circ \varphi^{-1}\right)_{\varphi(m)} \cdot v_{m}$. In our case we have only one chart and equivalence becomes trivial. Tangent vectors at $m$ can be considererd as derivatives on the germ $G(m)$ by defining

$$
v_{m}(f):=\left(D f_{m}\right) \cdot v_{m}=\left(D\left(f \circ \varphi^{-1}\right)_{\varphi(m)}\right) \cdot v_{m}
$$

Notice that the definition depends only on the equivalence class and not on the representant. The set of vectors tangent at $m$ defines the tangent space $T_{m}(M)$ of $M$ at $m$.

iii) The cotangent space $T_{m}^{\prime}(M)$ is the topological dual of $T_{m}(M)$, that is, the set of continuous linear functionals on $T_{m}(M)$. It is obviously isomorphic with $\mathcal{E}^{\prime}$, the topological dual of $\mathcal{E}$. Since our model space $\mathcal{E}$ is reflexive (it is a Hilbert space) we can naturally identify tangent and cotangent space (by the Riesz lemma) which also makes the definition of contravariant tensors less ambiguous. We will, however, not need them for what follows. Similarly, one defines the space of $p$-covariant tensors at $m \in M$ as the space of continuous $p$-linear forms on the $p$-fold tensor product of $T_{m}(M)$.

iv) So far the fact that $\mathcal{E}$ is a Banach manifold was not very crucial. But while the tangent bundle $T(M)=\cup_{m \in M} T_{m}(M)$ carries a natural manifold structure modelled on $\mathcal{E} \times \mathcal{E}$ for a general Fréchet space (or even locally convex space) $\mathcal{E}$ the cotangent bundle $T^{\prime}(M)=$ $\cup_{m \in M} T_{m}^{\prime}(M)$ carries a manifold structure only when $\mathcal{E}$ is a Banach space as one needs the inverse function theorem to show that each chart is not only a differentiable bijection but that also its inverse is differentiable. In our case again there is no problem. We define differentiable vector fields and $p$-covariant tensor fields as cross sections of the corresponding fibre bundles. 
v) A differential form of degree $p$ on $M$ or $p$-form is a cross section of the fibre bundle of completely skew continuous $p$-linear forms. Exterior product, pull-back, exterior differential, interior product with vector fields and Lie derivatives are defined as in the finite dimensional case.

Definition 3.1 Let $M$ be a differentiable manifold modelled on a Banach space $\mathcal{E}$. A weak respectively strong symplectic structure $\Omega$ on $M$ is a closed 2-form such that for all $m \in M$ the map

$$
\Omega_{m}: T_{m}(M) \mapsto T_{m}^{\prime}(M) ; v_{m} \rightarrow \Omega\left(v_{m}, .\right)
$$

is an injection respectively a bijection.

Strong symplectic structures are more useful because weak symplectic structures do not allow us to define Hamiltonian vector fields through the definition $D L+i_{\chi_{L}} \Omega=0$ for differentiable $L$ on $M$ and Poisson brackets through $\{f, g\}:=\Omega\left(\chi_{f}, \chi_{g}\right)$. Thus we define finally a strong symplectic structure for our case by

$$
\Omega\left((f, F),\left(f^{\prime}, F^{\prime}\right)\right):=\int_{\Sigma} d^{D} x\left[F_{i}^{a} f_{a}^{i \prime}-F_{i}^{a \prime} f_{a}^{i}\right](x)
$$

for any $(f, F),\left(f^{\prime}, F^{\prime}\right) \in \mathcal{E}$. To see that $\Omega$ is a strong symplectic structure we observe first that the integral kernel of $\Omega$ is constant so that $\Omega$ is clearly exact, so, in particular, closed. Next, let $\theta \in \mathcal{E}^{\prime} \equiv \mathcal{E}$. To show that $\Omega$ is a bijection it suffices to show that it is a surjection (injectivity follows trivially from linearity). We must find $(f, F) \in \mathcal{E}$ so that $\theta()=.\Omega((f, F),$.$) . Now by$ the Riesz lemma there exists $\left(f_{\theta}, F_{\theta}\right) \in \mathcal{E}$ such that $\theta()=.<\left(f_{\theta}, F_{\theta}\right), .>$ where $<., .>$ is the inner product induced by (3.4). Comparing (3.4) and (3.8) we see that we have achieved our goal provided that the functions

$$
F_{i}^{a}:=\rho^{a b} \sqrt{\operatorname{det}(\rho)} f_{b \theta}^{i}, f_{a}^{i}:=-\frac{\sigma_{a b}}{\sqrt{\operatorname{det}(\rho)}} F_{i \theta}^{b}
$$

are elements of $\mathcal{E}$. Inserting the definitions we see that this will be the case provided that the functions $\rho^{c d} \sigma_{c a} \sigma_{d b} / \sqrt{\operatorname{det}(\rho)}$ and $\operatorname{det}(\rho) \sigma_{c d} \rho^{c a} \rho^{d b} / \sqrt{\operatorname{det}(\sigma)}$ respectively fall off at least as $\sigma_{a b} / \sqrt{\operatorname{det}(\sigma)}$ and $\rho^{a b} \sqrt{\operatorname{det}(\rho)}$ respectively. In physical applications these metrics are usually chosen to be of the form $1+O(1 / r)$ where $r$ is an asymptotical radius function so that these conditions are certainly satisfied. Therefore, $(f, F) \in \mathcal{E}$ and our small lemma is established.

Let us compute the Hamiltonian vector field of a function $L$ on our $M$. By definition for all $(f, F) \in \mathcal{E}$ we have at $m=(A, E)$

$$
D L_{m} \cdot(f, F)=\int_{\Sigma} d^{D} x\left[\left(D L_{m}\right)_{i}^{a} f_{a}^{i}+\left(D L_{m}\right)_{a}^{i} F_{i}^{a}\right]=-\int_{\Sigma} d^{D} x\left[\left(\chi_{L m}\right)_{i}^{a} f_{a}^{i}-\left(\chi_{L m}\right)_{a}^{i} F_{i}^{a}\right.
$$

thus $\left(\chi_{L}\right)_{i}^{a}=-(D L)_{i}^{a}$ and $\left(\chi_{L}\right)_{a}^{i}=(D L)_{a}^{i}$. Obviously, this defines a bounded operator on $\mathcal{E}$ if and only if $L$ is differentiable. Finally, the Poisson bracket is given by

$$
\left\{L, L^{\prime}\right\}_{m}=\Omega\left(\chi_{L}, \chi_{L^{\prime}}\right)=\int_{\Sigma} d^{D} x\left[\left(D L_{m}\right)_{a}^{i}\left(D L_{m}^{\prime}\right)_{i}^{a}-\left(D L_{m}\right)_{i}^{a}\left(D L_{m}^{\prime}\right)_{a}^{i}\right]
$$

It is easy to see that $\Omega$ has the symplectic potential $\Theta$, a one-form on $M$, defined by

$$
\Theta_{m}((f, F))=\int_{\Sigma} d^{D} x E_{i}^{a} f_{a}^{i}
$$

since

$$
D \Theta_{m}\left((f, F),\left(f^{\prime}, F^{\prime}\right)\right):=\left(D\left(\Theta_{m}\right) \cdot(f, F)\right) \cdot\left(f^{\prime}, F^{\prime}\right)-\left(D\left(\Theta_{m}\right) \cdot\left(f^{\prime}, F^{\prime}\right)\right) \cdot(f, F)
$$


and $D E_{i}^{a}(x)_{m} \cdot(f, F)=F_{i}^{a}(x)$ as follows from the definition.

Coming back to the choice of $\mathcal{S}$, it will in general be a subspace of $\mathcal{E}$ so that (3.1) still converges. We can now compute the Poisson brackets between the functions $F(A), E(f)$ on $M$ and find

Remark :

$$
\left\{E(f), E\left(f^{\prime}\right)\right\}=\left\{F(A), F^{\prime}(A)\right\}=0,\{E(f), A(F)\}=F(f)
$$

In physicists notation one often writes $\left(D L_{m}\right)_{a}^{i}(x):=\frac{\delta L}{\delta A_{a}^{i}(x)}$ etc. and one writes the symplectic structure as $\Omega=\int d^{D} x D E_{i}^{a}(x) \wedge D A_{a}^{i}(x)$.

\subsection{New Symplectic Structure}

As outlined in the introduction we wish to define symplectic manifolds $\left(M_{\gamma}, \Omega_{\gamma}\right)$ for every $\gamma \in \Gamma$ which "in some sense come from $(M, \Omega)$ ". In order to do this we need besides graphs new extended objects associated with them. This is the topic of the following subsubsection. The class of graphs $\gamma$ that we have in mind consists of the set $\Gamma_{\sigma}^{\omega}$ of piecewise analytic, $\sigma$-finite graphs. These are graphs with an at most countable number of edges and such that for every compact subset $U$ of the locally compact manifold $\Sigma$ the restriction $U \cap \gamma$ is a piecewise analytic, finite graph in $\Gamma_{0}^{\omega}$. The precise definition of these graphs and their properties as well as the extension of the quantum kinematical framework to them needs the framework of the infinite tensor product of Hilbert spaces and will therefore be postponed to the first reference of [44]. For the rest of this paper one may without doing any mistake think of $\gamma$ as an element of $\Gamma_{0}^{\omega}$.

\subsubsection{Dual Polyhedral Decompositions}

Definition 3.2 A polyhedral decomposition $P$ of $\Sigma$ is a subdivision of $\Sigma$ into closed compact regions $\Delta$, that is $\Sigma=\cup_{\Delta \in P} \Delta$, each of which is diffeomorphic to a polyhedron in flat space and intersects any other polyhedron only in the set of points of their common boundary (of codimension at least one).

Note that we allow for decompositions with a countably infinite number of polyhedra in case that $\Sigma$ is not compact. We also do not insist that the decomposition be convex as this would require the choice of a background metric (rather of a diffeomorphism invariance class because convexity is defined by geodesity of curves which is a diffeomorphism invariant notion).

Definition 3.3 Let $P$ be a polyhedronal decomposition of $\Sigma$, pick any polyhedron $\Delta \in P$ and consider its boundary $\partial \Delta$.

i) A face $S$ of $\Delta$ (a maximal, connected, analytic subset of its boundary of codimension one) is called a "standard face" provided that

1) $S$ is a submanifold of $\Sigma$ of codimension one, diffeomorphic to a standard cube $[-1,1]^{D-1}$ in $\mathbb{R}^{D-1}$.

2) $S$ has no boundary, $\partial S=\emptyset$, i.e. it is open.

3) $S$ is contained in the domain of a chart of $\Sigma$.

4) $S$ is maximal, that is, there does not exist any $S^{\prime} \subset \partial \Delta$ properly containing $S$ which satisfies 1), 2) and 3).

ii) As $S$ is an open submanifold of $\Sigma$ of codimension one and for some $\Delta \in P, S \subset \partial \Delta$ is contained in the domain of a chart $(U, \varphi)$ of an atlas of $\Sigma$ there exists an open subset $V \subset U$ containing $S$, divided by $S$ into two halves and a diffeomorphism $\varphi^{\prime}$ that maps $V$, $S$ respectively to $\mathbb{R}^{D}$ and the hypersurface $x^{D}=0$ respectively. An orientation of $S$ is given by a choice of which of the half spaces or "sides" given by the set of points satisfying $x^{D}>0$ or $x^{D}<0$ we call "up" or "down".

iii) A polyhedronal decomposition of $\Sigma$ is said to be oriented provided the collection of all its standard faces have been oriented. 
This definition just formalizes the intuitive idea of a face of a polyhedron with a regular shape. Notice that a face is always shared by precisely two polyhedra of the decomposition. In $\mathrm{D}=1,2,3$ respectively simplicial polyhedra are given by closed lines, triangles and tetrahedra respectively and their faces are open points, lines and triangles respectively. The notion of an orientation is clearly independent of the chart employed. Also, faces are always orientable even if $\Sigma$ is not orientable and even if $\Sigma$ is orientable the orientation of a face can be opposite to the orientation induced from $\Sigma$ on $S$ (as a submanifold).

Definition 3.4 Given a graph $\gamma \in \Gamma$ we say that an oriented, polyhedral decomposition $P$ of $\Sigma$ is dual to $\gamma$ provided that:

1) Given an edge e of $\gamma$ there exists precisely one standard face $S_{e}$ from the collection of all faces of all the polyhedra of the decomposition which is intersected by $e$.

2) The edge e intersects $S_{e}$ transversally, that is, a) $\left\{p_{e}\right\}:=e \cap S_{e}$ consists of a single point which is an interior point of $e$ (it is necessarily an interior point of $S_{e}$ since $S_{e}$ is open) and b) there exists an open neighbourhood $V$ of $p_{e}$ and a diffeomorphism which maps $V$ to $\mathbb{R}^{D}, p_{e}$ to the origin of $\mathbb{R}^{D}, S_{e} \cap V$ to the plane $x^{D}=0$ and $e \cap V$ to the line $x^{1}=. .=x^{D-1}=0$.

3) The orientations of $S_{e}$ and e agree, that is, if under the diffeomorphism outlined in 2) the edge points into the half space $x^{D}>0$ or $x^{D}<0$ respectively then that half space corresponds to the "up" side of $S_{e}$.

4) The decomposition is irreducible, that is, one cannot reduce the number of polyhedra of the decomposition (by removing faces) without destroying at least one of the properties 1)-3).

Dual decompositions certainly exist in any dimension : A first example is given by a cubic lattice in any dimension (every vertex is 2D-valent) where we assume that $\Sigma$ has no boundary (periodic boundary conditions). The dual lattice is unique up to diffeomorphisms and corresponds to D-cubes around every vertex. A second example (again with periodic boundary conditions) is given by a simplicial lattice (every vertex is ( $\mathrm{D}+1$ )-valent) and corresponds to a simplicial decomposition with D-simplices around every vertex. Again the dual lattice is uniquely determined up to diffeomorphisms. Whether or not this uniqueness is a general feature of dual decompositions is not clear but we have the following.

Theorem 3.1 Given a graph $\gamma \in \Gamma$, a dual, oriented, polyhedral decomposition $P_{\gamma}$ of it exists and it is unique up to diffeomorphisms and up to the number of possibilities to obey the Euler (or Dehn-Sommerfeld in $D \neq 3$ ) relation for the various polyhedra under the reduction process.

Proof of Theorem 3.1 :

Existence :

Given a graph $\gamma$ consider for each vertex $v$ a closed neighbourhood $U_{v}$ of $v$ such that the various $U_{v}$ are mutually disjoint to begin with. Moreover, we can choose them to be closed balls such that their boundaries have the topology of the sphere. Now distort the $U_{v}$ along the edges $e$ incident at $v$ without changing their topology until for any edge $e$ with end points $v, v^{\prime}$ the $U_{v}, U_{v^{\prime}}$ intersect in precisely one point $p_{e}$ which is obviously an interior point of $e$, otherwise they are mutually non-intersecting. Now blow up the $U_{v}$ even more, keeping the $p_{e}$ fixed until each $U_{v}$ looks like a solid ball with a spherical boundary except for $n(v)$ cusps $S_{e}$ of the topology of cubes $[-1,1]^{D-1}$ with the $p_{e}$ as an interior point. $S_{e}$ is the only set in which $U_{v}, U_{v^{\prime}},\left\{v, v^{\prime}\right\}=\partial e$ intersect. By shifting the $p_{e}$ and varying the size of the cusps we can achieve that the $S_{e}$ are mutually non-intersecting, contained in the domain of a chart and intersect $e$ transversally. We can therefore equip them with an orientation that agrees with that of $e$. Altogether, the $U_{v}$ have now mutated to polyhedrons with $n(v)$ open, smooth standard faces $S_{e}, v \in \partial e$ and the additional closed connected component of its boundary consisting of $S_{v}:=\partial U_{v}-\cup_{v \in \partial e} S_{e}$. Finally, consider the polyhedronal decomposition of $\Sigma$ consisting of the $U_{v}$ and the remainder $\Sigma-\cup_{v} U_{v}$. This decomposition satisfies all the properties of a dual 
decomposition except, possibly, for the irreducibility requirement. To satisfy it, we remove the faces $S_{v}$ by letting the $S_{e}$ share their boundaries, as long as compatible with the Euler relation [54] between the number of connected components of all possible subcomplexes of a polyhedron. (For instance, in $\mathrm{D}=3$ the relation is given by $f=k-e+\chi$ where $f, k, e$ denotes the number of faces, links and corners of a polyhedron and $\chi$ is essentially the Euler characteristic of the manifold that the polyhedron triangulates). That this is always possible follows by the lemma of choice (or Zorn's lemma).

Uniqueness :

The constructive proof just given just has just fixed the topology of the final dual polyhedronal decomposition reached and is therefore unique at most up to diffemorphisms. Morover, the number of possible irreducible decompositions reachable obviously equals the number of possible solutions to the just mentioned Euler relation.

The non-uniqueness of $P_{\gamma}$ does not affect us because we will use only the $S_{e}$ which are determined up to diffeomorphisms.

Notice that on the other hand, if we are given an oriented cellular decomposition of $\Sigma$ into polyhedra with open faces, there is, up to diffeomorphism equivalence, only one graph such that the decomposition is dual to it. This follows easily from the fact that there is no choice but choosing an interior point of each polyhedron and connecting common faces between polyhedra with transversal edges running between the corresponding interior points with the obvious orientation.

From now on we pick for each graph $\gamma$ an oriented, polyhedral, dual decomposition $P_{\gamma}$. By theorem 3.1 we can do this in such a way that, if $\gamma \neq \gamma^{\prime}$ are diffeomorphic, then $P_{\gamma}, P_{\gamma^{\prime}}$ are diffeomorphic. Notice, however, that it is not possible to require that for any diffeomorphism $\varphi$ we have $P_{\varphi(\gamma)}=\varphi\left(P_{\gamma}\right)$ since there are many diffeomorphisms, say $\varphi_{1}, \varphi_{2}$ such that $\varphi_{1}(\gamma)=\varphi_{2}(\gamma)$ but $\varphi_{1}\left(P_{\gamma}\right) \neq \varphi_{2}\left(P_{\gamma}\right)$.

Also, a word of caution is appropriate with respect to refinements : The set $\Gamma$ is partially ordered by inclusion and for each pair $\gamma, \gamma^{\prime}$ there exists a bigger (refined) graph $\tilde{\gamma}$ containing both of them, for instance the graph $\gamma \cup \gamma^{\prime}$. However, in general it will not be true that there exists a refined graph such that $P_{\gamma}, P_{\gamma^{\prime}}$ are both contained in $P_{\tilde{\gamma}}$. This can happen only if the graphs under consideration have a high degree of symmetry as e.g. cubic lattices as one can show.

\subsubsection{The Graph Phase Space From the Continuum Phase Space}

We are now ready to derive a symplectic manifold $\left(M_{\gamma}, \Omega_{\gamma}\right)$ for each $\gamma \in \Gamma$ from the standard symplectic manifold $(M, \Omega)$ considered in the beginning of this section.

\section{Definition 3.5}

i) Let $S_{0}$ be the interior of the subset $[-1,1]^{D-1} \subset \mathbb{R}^{D-1}$ in the $x^{D}=0$ plane with normal orientation into the $x^{D}>0$ direction. Let $p_{0}=0$ be the origin in $\mathbb{R}^{D}$ and $e_{0}$ be the interval $[-1 / 2,1 / 2]$ of the $x^{D}$-axis. Let $x_{0} \in S_{0}$ and define $\rho_{0}\left(x_{0}\right)$ to be the straight line within $S_{0}$ connecting $p_{0}$ and $x_{0}$.

ii) Given a graph $\gamma$ and a dual polyhedronal decomposition $P_{\gamma}$, we call a collection of paths $\Pi_{\gamma}:=\left\{\rho_{e}(x) \subset S_{e} ; x \in S_{e}\right\}_{e \in E(\gamma)}$ adapted to $\gamma, P_{\gamma}$ provided there exists for each $e \in E(\gamma)$ an analytic diffeomorphism $\varphi_{e}: \mathbb{R}^{D} \mapsto \Sigma$ such that

$$
\left(e, S_{e}, p_{e}, x, \rho_{e}(x)\right)=\left(\varphi_{e}\left(e_{0}\right), \varphi_{e}\left(S_{0}\right), \varphi_{e}\left(p_{0}\right), \varphi_{e}\left(x_{0}\right), \varphi_{e}\left(\rho_{0}\left(x_{0}\right)\right)\right.
$$

We will denote the set of triples $\left(\gamma, P_{\gamma}, \Pi_{\gamma}\right)$ by $\mathcal{L}_{*}$ or $\mathcal{L}$ where $*=0, \sigma$ depends on the class of the graphs that we allow. The elements $l \in \mathcal{L}$ will be called structured graphs. We also use the 
notation $l=\left(\gamma(l), P_{\gamma}(l), \Pi_{\gamma}(l)\right)$.

iii) Given a structured graph l, let w.l.g. $p_{e}=e(1 / 2)$.

Then we define the following function on $(M, \Omega)$

$$
P_{i}^{e}(A, E):=-\frac{1}{N} \operatorname{tr}\left(\tau_{i} h_{e}(0,1 / 2)\left[\int_{S_{e}} h_{\rho_{e}(x)} * E(x) h_{\rho_{e}(x)}^{-1}\right] h_{e}(0,1 / 2)^{-1}\right)
$$

where $h_{e}(s, t)$ denotes the holonomy of $A$ along e between the parameter values $s<t, *$ denotes the Hodge dual, that is, $* E$ is a $(D-1)$-form on $\Sigma$ and $E^{a}:=E_{i}^{a} \tau_{i}$.

Notice that in contrast to similar variables used earlier in the literature 45 the function $P_{i}^{e}$ is gauge covariant. Namely, under gauge transformations it transforms as $P^{e} \mapsto g(e(0)) P^{e} g(e(0))^{-1}$, the price to pay being that $P^{e}$ depends on both $A$ and $E$ and not only on $E$. As we will see shortly, this is actually an advantage. Of course, the notation (3.15) is abusing as $P^{e}$ not only depends on $e$ but actually on $S_{e}, \rho_{e}(x), x \in S_{e}$. In the sequel, unless confusion can arise we will continue abusing notation and write $\gamma$ instead of $l$.

The problem with the functions $h_{e}(A)$ and $P_{i}^{e}(A, E)$ on $M$ is that they are not differentiable on $M$, that is, $D h_{e}, D P_{i}^{e}$ are nowhere bounded operators on $\mathcal{E}$ as one can easily see. The reason for this is, of course, that these are functions on $M$ which are not properly smeared with functions from $\mathcal{S}$, rather they are smeared with distributional test functions with support on $e$ or $S_{e}$ respectively. Nevertheless one would like to base the quantization of the theory on these functions as basic variables because of their gauge and diffeomorphism covariance. Indeed, under diffeomorphisms the structured graph $l$ is simply replaced by

$$
\varphi^{-1}(l)=\left(\varphi^{-1}(e), \varphi^{-1}\left(S_{e}\right),\left\{\varphi^{-1}\left(\rho_{e}(x)\right) ; x \in S_{e}\right\}\right)_{e \in E(\gamma)}
$$

which is a structured graph again and in this sense $h_{e} \mapsto h_{\varphi^{-1}(e)}, P_{i}^{e} \mapsto P_{i}^{\varphi^{-1}(e)}$. Furthermore, their quantizations are properly represented on the Hilbert space described in section 2 as we will see. The fact that the smearing dimensions of $h_{e}$ and $P_{i}^{e}$ add to $D$ raises some hope that one can still derive a bona fide Poisson algebra among these variables. We therefore define

Definition 3.6 The set of pairs $\left(h_{e}(A), P_{i}^{e}(A, E)\right)_{e \in E(\gamma)}$ as $(A, E)$ varies over $M$ will be denoted by $\bar{M}_{\gamma \mid M}$. We also define $\bar{M}_{\gamma}=(G \times \operatorname{Lie}(G))^{|E(\gamma)|}$.

It is easy to see that $\bar{M}_{\gamma \mid M}$ is generically a proper subset of $\bar{M}_{\gamma}$. Indeed, since the edges $e$ are mutually disjoint among each other except for the vertices we can find a smooth connection with support in disjoint open neighbourhoods $U_{e}$, one for each $e$, such that $e \cap U_{e}$ is an open segment of $e$. The holonomy along those segments can be given independent values since we can vary the behaviour of $A$ in each $U_{e}$ independently and arbitrarily without destroying smoothness. Similar considerations hold for the momenta $P_{i}^{e}$. The range of these values is, however, constrained by the boundary conditions imposed by the fact that $(A, E)$ are points in a classical phase space subject to fall-off conditions. The bar in the notation $\bar{M}_{\gamma}$ accounts for the fact that the points of $\bar{M}_{\gamma}$ do not satisfy such regularity assumptions similar as in the case of $\overline{\mathcal{A}}_{\gamma}$.

We equip a subset $M_{\gamma}$ of $\bar{M}_{\gamma}$ with the following natural topology : Let $\left(u_{i}, \phi_{i}\right)_{i \in I}$ be an atlas of $G$ where $I$ is a finite index set (always possible since $G$ is compact). For instance the $u_{i}$ could be preimages of open sets in $\mathbb{R}^{\operatorname{dim}(G)}$ under the exponential map which is locally a diffeomorphism between $G$ and $\operatorname{Lie}(G)$. Since $\operatorname{Lie}(G)$ is topologically trivial we can construct an atlas of $G \times \operatorname{Lie}(G)$ by $\left(U_{i}=u_{i} \times \operatorname{Lie}(G), \Phi_{i}=\phi_{i} \times\right.$ id $)$ where id denotes the identity map of $\mathbb{R}^{\operatorname{dim}(G)}$. Then $M_{\gamma}$ can be given the differentiable structure defined by the atlas

$$
\left(\times_{e \in E(\gamma)} U_{i_{e}}, \times_{e \in E(\gamma)} \Phi_{i_{e}}\right)_{i_{e} \in I}
$$


which displays $M_{\gamma}$ as a Banach manifold modelled on $\mathcal{E}_{\gamma}=\mathbb{R}^{2 \operatorname{dim}(G)|E(\gamma)|} . \mathcal{E}_{\gamma}$ is equipped with the norm

$$
\left\|\left\{x_{e}, y_{e}\right\}_{e \in E(\gamma)}\right\|_{\rho, \sigma}:=\sqrt{\sum_{e \in E(\gamma)}\left[\rho_{e}^{i j} x_{e}^{i} x_{e}^{j}+\sigma_{i j}^{e} y_{e}^{i} y_{e}^{j}\right]}
$$

where $\rho_{e}^{i j}, \sigma_{i j}^{e}$ are metrics of Euclidean signature for each $e$. Obviously, then $M_{\gamma}$ is a proper subset of $\bar{M}_{\gamma}$.

Remark :

A connection between (3.18) and (3.2) can be given on certain graphs as follows : The idea is that (3.18) is a discretization of (3.2) so that they eqaul each other in the limit of an infinitely fine graph. Consider for simplicity $\Sigma=\mathbb{R}^{3}$ (the generalization to arbitrary $\Sigma$ is straightforward with the tools of the next subsection) and consider a lattice $\gamma$ of regular cubic topology. Then edges can be labelled as $t \mapsto e_{I}(v, t)$ where $v=e_{I}(v, 0)$ is a vertex and $I=1, . ., D$. Let edges be images of the interval $t \in[0, \epsilon]$ and define $Y_{I}^{a}(v)=\dot{e}_{I}^{a}(v, 0), n_{a}^{I}(v)=$ $\frac{1}{(D-1) !} \epsilon_{a b_{1} . . b_{D-1}} \epsilon^{I J_{1} . . J_{D-1}} Y_{J_{1}}^{b_{1}}(v) . . Y_{J_{D-1}}^{b_{D-1}}(v)$. We now choose $\rho_{i j}^{e}=\delta_{i j} f(e), \sigma_{e}^{i j}=\delta^{i j} g(e)$ for some functions $f, g: E(\gamma) \mapsto \mathbb{R}$ and choose the $e_{I}(v)$ in such a way that

$\left.\sum_{I} f\left(e_{I}(v)\right)\right) Y_{I}^{a}(v) Y_{I}^{b}(v)=\epsilon^{D-2}\left(\sqrt{\operatorname{det}(\rho)} \rho^{a b}\right)(v)$ and $\sum_{I} g\left(e_{I}(v)\right) n_{a}^{I}(v) n_{b}^{I}(v)=\epsilon^{-(D-2)}\left(\sigma_{a b} / \sqrt{\operatorname{det}(\sigma)}\right)(v)$

the metrics of $(3.2)$ result. It is then easy to see that with $x_{i}^{e}:=-\frac{2}{N} \operatorname{tr}\left(\tau_{i} h_{e}(A) h_{e}\left(A^{\prime}\right)^{-1}\right), y_{e}^{i}:=$ $P_{i}^{e}(A, E)-P_{i}^{e}\left(A^{\prime}, E^{\prime}\right)$ the resulting metric (3.18) converges to the metric (3.2).

In order to proceed and to give $M_{\gamma}$ a symplectic structure derived from that of $M$ one must regularize the elementary functions so that one can use the symplectic structure $\Omega$, then study the limit in which the regulator is removed and hope that the result is a well-defined symplectic structure $\Omega_{\gamma}$. We choose the following regularization :

Given an edge $e$ we define a tube $T^{e}$ around $e$ to be a foliation of $D-1$ dimensional surfaces which are topologically discs. Let $f_{\epsilon}: D \mapsto T_{\epsilon}^{e}$ be a one-parameter family of smooth functions which tends to the $\delta$-distribution on the unit disc $D$. Recall that the holonomy of a smooth connection can be written as the path ordered exponential

$$
h_{e}(A)=1+\sum_{n=1}^{\infty} \int_{0}^{1} d t_{n} \int_{0}^{t_{n}} d t_{n-1} . . \int_{0}^{t_{2}} d t_{1} A\left(t_{1}\right) . . A\left(t_{n}\right)
$$

where $A(t)=\dot{e}^{a}(t) A_{a}^{i}(e(t)) \tau_{i} / 2$. We define the holonomy smeared over the tube $T_{\epsilon}^{e}$ by

$$
h_{e}^{\epsilon}(A)=1+\sum_{n=1}^{\infty} \int_{0}^{1} d t_{n} \int_{0}^{t_{n}} d t_{n-1} . . \int_{0}^{t_{2}} d t_{1} \int_{D} d^{D-1} y_{n} f_{\epsilon}\left(y_{n}\right) . . \int_{D} d^{D-1} y_{1} f_{\epsilon}\left(y_{1}\right) A_{y_{1}}\left(t_{1}\right) . . A_{y_{n}}\left(t_{n}\right)
$$

where $A_{y}(t)=\dot{e}_{y}^{a}(t) A_{a}^{i}\left(e_{y}(t)\right) \tau_{i} / 2$ and $D \times[0,1] \mapsto T^{e} ;(y, t) \mapsto e_{y}(t)$ defines the tube $T^{e}$ with the convention that $e_{0}(t)=e(t)$.

Likewise, let $R^{e}$ be a region foliated by surfaces diffeomorphic to $S^{e}$. Let $X: V \mapsto$ $\left.S^{e} ; u:=\left(u_{1}, . ., u_{D-1}\right)\right) \mapsto X(u)$ be a parameterization of $S^{e}$ where $V$ is an open submanifold of $\mathbb{R}^{D}$ of dimension $D-1$. Furhermore, let $[-1,1] \times V \mapsto R^{e} ;(s, u) \mapsto X_{s}(u)$ define $R^{e}$ with the convention that $X_{0}(u)=X(u)$. Let $g_{\epsilon}$ be a one parameter family of smooth functions which tends to the $\delta$-distribution on the interval $[-1,1]$ as $\epsilon \rightarrow 0$. Also, define $\rho_{e}^{s}(x)$ to be paths in $X_{s}(V)=S_{e}^{s}$ between $p_{e}(s)=X_{s}(0)=e \cap X_{s}(V)$ and $x \in X_{s}(V)$ and let $e_{s}$ be a reparameterization of $e$ such that $e_{s}(1 / 2)=p_{e}^{s}$. Here we assume w.l.g. that all the surfaces $S_{e}^{s}, s \in[-1,1]$ satisfy the conditions that the surface $S_{e}$ has to satisfy. We can now define

$$
P_{i, s}^{e}(A, E):=-\frac{1}{N} \operatorname{tr}\left(\tau_{i} h_{e_{s}}(0,1 / 2)\left[\int_{S_{e}^{s}} h_{\rho_{e_{s}}(x)} * E(x) h_{\rho_{e_{s}}(x)}^{-1}\right] h_{e_{s}}(0,1 / 2)^{-1}\right)
$$


and then

$$
P_{i}^{e \epsilon}:=\int_{-1}^{1} d s g_{\epsilon}(s) P_{i, s}^{e}
$$

Notice that the holonomies involved in (3.22) remain unsmeared as compared to (3.20). We could improve this by an additional smearing, preserving gauge covariance, but it would just blow up the subsequent calculations and would not change the end result. The careful reader is invited to fill in the missing details.

Apart from these details, the functions (3.20) and (3.22) are now written as functions of the variables $F(A), E(f)$ where $F, f$ are of the form $F_{i}^{a}(x)=\chi_{T e}(x)\left(f_{\epsilon}(y) \dot{e}_{y}^{a}(t) \delta_{i}^{j}\right)_{x=e_{y}(t)}$ and $f_{a}^{i}(x)=\chi_{R^{e}}(x)\left(g_{\epsilon}(s) \epsilon_{a b_{1} . . b_{D-1}} X_{s, u_{1}}^{b 1}(u) . . X_{s, u_{D-1}}^{b 2}(u) \delta_{i}^{j}\right)_{x=X_{s}(u)}$ for some $j$ and are thus certainly elements of $\mathcal{S}$. It follows that the smeared functions are functionally differentiable. Moreover, by construction, the smeared objects converge pointwise on $M$ to the unsmeared objects, that is

$$
\left.\lim _{\epsilon \rightarrow 0} \mid\left(h_{e}^{\epsilon}(A)\right)_{A B}-\left(h_{e}(A)\right)_{A B}\right)\left|=\lim _{\epsilon \rightarrow 0}\right| P_{i}^{e \epsilon}(A, E)-P_{i}^{e}(A, E) \mid=0
$$

for all $(A, E) \in M, i=1, . . \operatorname{dim}(G), A, B$ where $A, B,$. denote group indices.

Theorem 3.2 The smeared variables allow us to define the following bracket $\{., .\}_{\gamma}$ on $M_{\gamma}$ :

$$
\begin{aligned}
\left\{h_{e}, h_{e^{\prime}}\right\}_{\gamma} & :=\lim _{\epsilon_{1}, \epsilon_{2} \rightarrow 0}\left\{h_{e}^{\epsilon_{1}}, h_{e^{\prime}}^{\epsilon_{2}}\right\}=0 \\
\left\{P_{i}^{e}, h_{e^{\prime}}\right\}_{\gamma} & :=\lim _{\epsilon_{1} \rightarrow 0} \lim _{\epsilon_{2} \rightarrow 0}\left\{P_{i}^{e \epsilon_{1}}, h_{e^{\prime}}^{\epsilon_{2}}\right\}=\delta_{e^{\prime}}^{e} \frac{\tau_{i}}{2} h_{e} \\
\left\{P_{i}^{e}, P_{j}^{e^{\prime}}\right\}_{\gamma} & :=\lim _{\epsilon_{1}, \epsilon_{2} \rightarrow 0}\left\{P_{i}^{e \epsilon_{1}}, P_{j}^{e^{\prime} \epsilon_{2}}\right\}=-\delta^{e e^{\prime}} f_{i j}{ }^{k} P_{k}^{e}
\end{aligned}
$$

where $\{.,$.$\} is the bracket on M$ and convergence is meant here and in what follows to be pointwise on $M$.

Notice that we do not yet call $\{., .\}_{\gamma}$ a Poisson bracket since we must check that it qualifies as a (strong) symplectic structure. This we will do in a separate step.

Proof of Theorem 3.2 :

[1.]

Recalling (3.13) the first identity (3.24) follows easily from the fact that $\left\{h_{e}^{\epsilon_{1}}, h_{e^{\prime}}^{\epsilon_{2}}\right\}=0$ at every finite $\epsilon_{1}, \epsilon_{2}$. It is for this reason that we do not have to smear the $h_{\rho_{e}^{s}(x)}, h_{e_{s}}$ involved in (3.21) in addition to $E$ in order to define the brackets, there would be no extra contribution due to this fact.

[2.]

The second identity (3.25) is significantly more involved. We first prove the following lemma.

Lemma 3.1 For any $f_{a}^{i} \in \mathcal{S}$ and any path e we have

$$
\left\{E(f), h_{e}\right\}:=\lim _{\epsilon \rightarrow 0}\left\{E(f), h_{e}^{\epsilon}\right\}=\int_{0}^{1} d t \dot{e}^{a}(t) f_{a}^{i}(e(t)) h_{e}(0, t) \frac{\tau_{i}}{2} h_{e}(t, 1)
$$

Proof of Lemma 3.1:

We have by definition

$$
\begin{aligned}
\left\{E(f), h_{e}^{\epsilon}(A)\right\}= & \sum_{n=1}^{\infty} \int_{0}^{1} d t_{n} \int_{0}^{t_{n}} d t_{n-1} . . \int_{0}^{t_{2}} d t_{1} \int_{D} d^{D-1} y_{n} f_{\epsilon}\left(y_{n}\right) . . \int_{D} d^{D-1} y_{1} f_{\epsilon}\left(y_{1}\right) \times \\
& \times \sum_{k=1}^{n} A_{y_{1}}\left(t_{1}\right) . .\left\{E(f), A_{y_{k}}\left(t_{k}\right)\right\} . . A_{y_{n}}\left(t_{n}\right) \\
= & \sum_{n=1}^{\infty} \int_{0}^{1} d t_{n} \int_{0}^{t_{n}} d t_{n-1} . . \int_{0}^{t_{2}} d t_{1} \int_{D} d^{D-1} y_{n} f_{\epsilon}\left(y_{n}\right) . . \int_{D} d^{D-1} y_{1} f_{\epsilon}\left(y_{1}\right) \times \\
& \times \sum_{k=1}^{n} f_{a}^{i}\left(e_{y_{k}}\left(t_{k}\right)\right) \dot{e}_{y_{k}}^{a}\left(t_{k}\right) A_{y_{1}}\left(t_{1}\right) . . \frac{\tau_{i}}{2} . . A_{y_{n}}\left(t_{n}\right)
\end{aligned}
$$


Relabel $T_{1}=t_{1}, . ., T_{k-1}=t_{k-1}, t=t_{k}, T_{k}=t_{k+1}, . ., T_{n-1}=t_{n}$ and $z_{1}=y_{1}, . ., z_{k-1}=y_{k-1}, y=$ $y_{k}, z_{k}=y_{k+1}, . ., z_{n-1}=y_{n}$ then (3.28) becomes

$$
\begin{aligned}
\left\{E(f), h_{e}^{\epsilon}(A)\right\}= & \sum_{n=1}^{\infty} \sum_{k=1}^{n} \int_{0}^{1} d T_{n-1} \int_{0}^{T_{n-1}} d T_{n-2} . . \int_{0}^{T_{k+1}} d T_{k} \int_{0}^{T_{k}} d t \int_{0}^{t} d T_{k-1} . . \int_{0}^{T_{2}} d T_{1} \times \\
& \times \int_{D} d^{D-1} y f_{\epsilon}(y) f_{a}^{i}\left(e_{y}(t)\right) \dot{e}_{y}^{a}(t) \int_{D} d^{D-1} z_{n-1} f_{\epsilon}\left(z_{n-1}\right) . . \int_{D} d^{D-1} z_{1} f_{\epsilon}\left(z_{1}\right) \times \\
& \times \sum_{k=1}^{n} A_{z_{1}}\left(T_{1}\right) . \frac{\tau_{i}}{2} . . A_{z_{n-1}}\left(T_{n-1}\right) \\
= & \sum_{n=1}^{\infty} \sum_{k=1}^{n} \int_{0}^{1} d t \int_{D} d^{D-1} y f_{\epsilon}(y) f_{a}^{i}\left(e_{y}(t)\right) \dot{e}_{y}^{a}(t) \times \\
& \times \int_{t}^{1} d T_{n-1} \int_{t}^{T_{n-1}} d T_{n-2} . \cdot \int_{t}^{T_{k+1}} d T_{k} \int_{0}^{t} d T_{k-1} \int_{0}^{T_{k-1}} d T_{k-2} . . \int_{0}^{T_{2}} d T_{1} \times \\
& \times \int_{D} d^{D-1} z_{n-1} f_{\epsilon}\left(z_{n-1}\right) . . \int_{D} d^{D-1} z_{1} f_{\epsilon}\left(z_{1}\right) \sum_{k=1}^{n} A_{z_{1}}\left(T_{1}\right) . . \frac{\tau_{i}}{2} . . A_{z_{n-1}}\left(T_{n-1} \gamma 3.29\right)
\end{aligned}
$$

where in the last step we have used the fact that $1 \geq T_{n-1} \geq . . \geq T_{k} \geq t \geq T_{k-1} \geq . . \geq T_{1} \geq 0$ in order to make the range of integration of $t$ independent of the $T_{k}$. We can now easily take the limit $\epsilon \rightarrow 0$ with the result

$$
\begin{aligned}
& \sum_{n=1}^{\infty} \sum_{k=1}^{n} \int_{0}^{1} d t f_{a}^{i}(e(t)) \dot{e}^{a}(t) \int_{t}^{1} d T_{n-1} \int_{t}^{T_{n-1}} d T_{n-2} . . \\
& . . \int_{t}^{T_{k+1}} d T_{k} \int_{0}^{t} d T_{k-1} \int_{0}^{T_{k-1}} d T_{k-2 \cdot .} \int_{0}^{T_{2}} \sum_{k=1}^{n} A_{z_{1}}\left(T_{1}\right) . . \frac{\tau_{i}}{2} . . A_{z_{n-1}}\left(T_{n-1}\right)
\end{aligned}
$$

and writing out the path product identity for holonomies $h_{e}(0, t) h_{e}(t, 1)=h_{e}(0,1)=h_{e}$ in the path ordered form (3.19) this collapses indeed to (3.27).

Let us now prove the second identity (3.25). Let us write $P_{i}^{e \epsilon}$ in the form $E(f)$ by choosing

$$
\begin{aligned}
\left(f_{i}^{\epsilon}\right)_{a}^{j}(x)= & -\int_{-1}^{1} d s \int_{V} d^{D-1} u \delta\left(x, X_{s}(u)\right) \frac{1}{N} g_{\epsilon}(s) \epsilon_{a b_{1} . . b_{D-1}} X_{s, u_{1}}^{b_{1}}(u) . . X_{s, u_{D-1}}^{b_{D-1}}(u) \times \\
& \times \operatorname{tr}\left(\tau_{i} h_{e_{s}}(0,1 / 2) h_{\rho_{e_{s}}\left(X_{s}(u)\right)} \tau_{j} h_{\rho_{e_{s}}\left(X_{s}(u)\right)}^{-1} h_{e_{s}}(0,1 / 2)^{-1}\right)
\end{aligned}
$$

From [1.] it is clear that, although $f_{a}^{j}$ depends on $A$, as far as (3.25) is concerned, we can treat it as a numerical function. By Lemma 3.1 we then have

$$
\left\{P_{i}^{e}, h_{e^{\prime}}\right\}_{\gamma}=\lim _{\epsilon \rightarrow 0}\left\{E\left(f_{i}^{\epsilon}\right), h_{e^{\prime}}\right\}=\lim _{\epsilon \rightarrow 0} \int_{0}^{1} d t \dot{e}^{a \prime}(t)\left(f_{i}^{\epsilon}\right)_{a}^{j}\left(e^{\prime}(t)\right) h_{e^{\prime}}(0, t) \frac{\tau_{j}}{2} h_{e^{\prime}}(t, 1)
$$

Suppose first that $e \neq e^{\prime}$. Then, no matter how complicated $\gamma, P_{\gamma}$ look, for sufficiently small $\epsilon$ the edge $e^{\prime}$ does not intersect the region $R_{\epsilon}^{e}$ and thus (3.32) vanishes. If $e=e^{\prime}$ then $e$ intersects $R_{\epsilon}^{e}$ for any value of $\epsilon$ and we find

$$
\begin{aligned}
& \left\{P_{i}^{e}, h_{e}\right\}_{\gamma}=-\lim _{\epsilon \rightarrow 0} \frac{1}{N} \int_{-1}^{1} d s g_{\epsilon}(s) \int_{0}^{1} d t \int_{V} d^{D-1} u \delta\left(e(t), X_{s}(u)\right) \dot{e}^{a}(t) \times \\
& \times \epsilon_{a b_{1} . . b_{D-1}} X_{s, u_{1}}^{b_{1}}(u) . . X_{s, u_{D-1}}^{b_{D-1}}(u) \operatorname{tr}\left(\tau_{i} h_{e_{s}}(0,1 / 2) h_{\rho_{e_{s}}\left(X_{s}(u)\right)} \times\right. \\
& \left.\times \tau_{j} h_{\rho_{e_{s}}\left(X_{s}(u)\right)}^{-1} h_{e_{s}}(0,1 / 2)^{-1}\right) h_{e}(0, t) \frac{\tau_{j}}{2} h_{e}(t, 1)
\end{aligned}
$$

At fixed $s$ the only contribution of the integral over $(t, u) \in[0,1] \times V$ comes from the value $\left(t=t_{s}, u=0\right)$ since $S_{e}^{s}$ and $e$ intersect in the only point $p_{e}^{s}$ which is an interior point of $[0,1] \times V$ 
for sufficiently small $\epsilon$. Here $t_{s}$ is defined by $e_{s}(1 / 2)=e\left(t_{s}\right)$. By definition of the orientation of the $X_{s}(V)$ we know that $\dot{e}^{a}(t) \epsilon_{a b_{1} . . b_{D-1}} X_{s, u_{1}}^{b_{1}}(u) . . X_{s, u_{D-1}}^{b_{D-1}}(u)>0$ at $\left(t=t_{s}, u=0\right)$. Since $\rho_{e_{s}}\left(p_{e}^{s}\right)=p_{e}^{s}$, 3.33) collapses to

$$
\left\{P_{i}^{e}, h_{e^{\prime}}\right\}_{\gamma}=-\lim _{\epsilon \rightarrow 0} \frac{1}{N} \int_{-1}^{1} d s g_{\epsilon}(s) \operatorname{tr}\left(\tau_{i} h_{e_{s}}(0,1 / 2) \tau_{j} h_{e_{s}}(0,1 / 2)^{-1}\right) h_{e_{s}}(0,1 / 2) \frac{\tau_{j}}{2} h_{e_{s}}(1 / 2,1)
$$

Since the integrand depends continuously on $s$ for any smooth connection we see that pointwise

$$
\left\{P_{i}^{e}, h_{e^{\prime}}\right\}_{\gamma}=-\frac{1}{N} \operatorname{tr}\left(\tau_{i} h_{e}(0,1 / 2) \tau_{j} h_{e}(0,1 / 2)^{-1}\right) h_{e}(0,1 / 2) \frac{\tau_{j}}{2} h_{e}(1 / 2,1)
$$

Now consider the matrix $C_{i}=h_{e}(0,1 / 2)^{-1} \tau_{i} h_{e}(0,1 / 2)$ which is an element of the Lie algebra of $G$ because it is simply the transform of $\tau_{i}$ under the action of $h_{e}(0,1 / 2)^{-1}$ in the adjoint representation of $G$ on $\operatorname{Lie}(G)$. Thus we can expand $C_{i}$ in the basis $\tau_{j}$, resulting in $C_{i}=$ $-\operatorname{tr}\left(C_{i} \tau_{j}\right) \tau_{j} / N$. Inserting this identity into (3.35) gives the result claimed.

[3.]

Let us now turn to the final third identity (3.26). It is clear that for $e \neq e^{\prime}$ and $\epsilon_{1}, \epsilon_{2}$ sufficiently small the regions $R_{e}^{\epsilon_{1}}, R_{e^{\prime}}^{\epsilon_{2}}$ are disjoint in which case the brackets vanish. Thus we only need to be concerned with the case $e=e^{\prime}$. Let us again use the convention (3.31) and let us introduce the notation that for a function $f$ depending on $(A, E)$, the function $f$ is numerically equal to $f$ but $\tilde{f}$ is considered to be independent of $(A, E)$. In other words, $\tilde{f}$ drops out of Poisson brackets on $M$ but $f$ does not necessarily. Then we have by the Leibniz rule for $\Omega$

$$
\begin{aligned}
& \left\{P_{i}^{e \epsilon_{1}}, P_{j}^{e \epsilon_{2}}\right\}=\left\{E\left(f_{i}^{\epsilon_{1}}\right), E\left(f_{j}^{\epsilon_{2}}\right)\right\} \\
= & \left\{E\left(\tilde{f}_{i}^{\epsilon_{1}}\right), E\left(\tilde{f}_{j}^{\epsilon_{2}}\right)\right\}+\left\{E\left(\tilde{f}_{i}^{\epsilon_{1}}\right), \tilde{E}\left(f_{j}^{\epsilon_{2}}\right)\right\}+\left\{\tilde{E}\left(f_{i}^{\epsilon_{1}}\right), E\left(\tilde{f}_{j}^{\epsilon_{2}}\right)\right\}+\left\{\tilde{E}\left(f_{i}^{\epsilon_{1}}\right), \tilde{E}\left(f_{j}^{\epsilon_{2}}\right)\right\}
\end{aligned}
$$

The first term drops out by definition of $\Omega$ (recall (3.13)) and the fourth by [1.] so that only the second and third term survive. More explicitly

$$
\left.\left.\left\{P_{i}^{e \epsilon_{1}}, P_{j}^{e \epsilon_{2}}\right\}=\int_{\Sigma} d^{3} x E_{k}^{a}(x)\left[\left\{E\left(\tilde{f}_{i}^{\epsilon_{1}}\right),\left(f_{j}^{\epsilon_{2}}\right)_{a}^{k}(x)\right)\right\}-\left\{E\left(\tilde{f}_{j}^{\epsilon_{2}}\right),\left(f_{i}^{\epsilon_{1}}\right)_{a}^{k}(x)\right)\right\}\right]
$$

In order to compute (3.37) in the limit $\epsilon_{1}, \epsilon_{2} \rightarrow 0$ we have to consider two types of terms, namely $\left\{E\left(\tilde{f}_{i}^{\epsilon}\right), h_{\rho_{e_{s}}\left(X_{s}(u)\right)}\right\}$ and $\left\{E\left(\tilde{f}_{i}^{\epsilon}\right), h_{e_{s}}(0,1 / 2)\right\}$ The first term is given, according to Lemma 3.1, by

$$
\begin{aligned}
& \int_{0}^{1} d t \dot{\rho}_{e_{s}}^{a}\left(X_{s}(u), t\right)\left(\tilde{f}_{i}^{\epsilon}\right)_{a}^{j}\left(\rho_{e_{s}}\left(X_{s}(u)\right) h_{\rho_{e_{s}}\left(X_{s}(u)\right)}(0, t) \frac{\tau_{j}}{2} h_{\rho_{e_{s}}\left(X_{s}(u)\right)}(t, 1)\right. \\
= & -\frac{1}{N} \int_{0}^{1} d t h_{\rho_{e_{s}}\left(X_{s}(u)\right)}(0, t) \frac{\tau_{j}}{2} h_{\rho_{e_{s}}\left(X_{s}(u)\right)}(t, 1) \int_{-1}^{1} d r g_{\epsilon}(r) \int_{V} d^{D-1} v \times \\
& \times \delta\left(\rho_{e_{s}}\left(X_{s}(u), t\right), X_{r}(v)\right) \dot{\rho}_{e_{s}}^{a}\left(X_{s}(u), t\right) \epsilon_{a b_{1} . . b_{D-1}} X_{r, v_{1}}^{b_{1}}(v) . . X_{r, v_{D-1}}^{b_{D-1}}(v) \times \\
& \times \operatorname{tr}\left(\tau_{i} h_{e_{r}}(0,1 / 2) h_{\rho_{e_{r}}\left(X_{r}(v)\right)} \tau_{j} h_{\rho_{e_{r}}\left(X_{r}(v)\right)}^{-1} h_{e_{r}}(0,1 / 2)^{-1}\right)
\end{aligned}
$$

Using the fact that $r \mapsto X_{r}(V)$ defines a foliation $R_{e}$ of surfaces diffeomorphic to $S_{e}$ we see that the integral over $r, v$ is supported at the interior point $r=(s, v(s, t, u))$ of $[-1,1] \times V$ where $X_{s}(v(s, t, u))=\rho_{e_{s}}\left(X_{s}(u), t\right)$. The integral can be performed with the result

$$
\begin{aligned}
& -\frac{1}{N} \int_{0}^{1} d t h_{\rho_{e_{s}}\left(X_{s}(u)\right)}(0, t) \frac{\tau_{j}}{2} h_{\rho_{e_{s}}\left(X_{s}(u)\right)}(t, 1) g_{\epsilon}(s) \times \\
& \times\left[\frac{\dot{\rho}_{e_{s}}^{a}\left(X_{s}(u), t\right) \epsilon_{a b_{1} . . b_{D-1}} X_{r, v_{1}}^{b_{1}}(v) . . X_{r, v_{D-1}}^{b_{D-1}}(v)}{\left|\epsilon_{a b_{1} . . b_{D-1}} X_{r, r}^{a} X_{r, v_{1}}^{b_{1}}(v) . . X_{r, v_{D-1}}^{b_{D-1}}(v)\right|} \times\right. \\
& \left.\times \operatorname{tr}\left(\tau_{i} h_{e_{r}}(0,1 / 2) h_{\rho_{e_{r}}\left(X_{r}(v)\right)} \tau_{j} h_{\rho_{e_{r}}\left(X_{r}(v)\right)}^{-1} h_{e_{r}}(0,1 / 2)^{-1}\right)\right]_{r=s, v=v(s, t, u)}
\end{aligned}
$$


Notice that the denominator in (3.39) is bounded away from zero as the curve $s \mapsto X_{s}(v)$ for any fixed $v$ is transversal to $X_{s}(V)$. Now $\rho_{e_{s}}\left(X_{s}(u), t\right)=\left(X_{r}(v)\right)_{r=s, v=v(s, t, u)}$ for any $t \in[0,1]$, thus $\dot{\rho}_{e_{s}}\left(X_{s}(u), t\right)=\sum_{k=1}^{D-1}\left(X_{r, v_{k}}(v)\right)_{r=s, v=v(s, t, u)} \frac{d v_{k}(s, t, u)}{d t}$, thus the integrand of (3.39) vanishes for any finite $\epsilon$.

Thus there will be no contribution from the holonomies along the $\rho_{e_{s}}\left(X_{s}(u)\right)$ and we can focus on the second term $\left\{E\left(\tilde{f}_{i}^{\epsilon}\right), h_{e_{s}}(0,1 / 2)\right\}$ mentioned. Again, according to Lemma 3.1, it is given by

$$
\begin{aligned}
& \int_{0}^{1 / 2} d t \dot{e}_{s}^{a}(t)\left(\tilde{f}_{i}^{\epsilon}\right)_{a}^{j}\left(e_{s}(t)\right) h_{e_{s}}(0, t) \frac{\tau_{j}}{2} h_{e_{s}}(t, 1 / 2) \\
= & -\frac{1}{N} \int_{0}^{1 / 2} d t h_{e_{s}}(0, t) \frac{\tau_{j}}{2} h_{e_{s}}(t, 1 / 2) \int_{-1}^{1} d r g_{\epsilon}(r) \int_{V} d^{D-1} v \delta\left(e_{s}(t), X_{r}(v)\right) \dot{e}_{s}^{a}(t) \times \\
& \times \epsilon_{a b_{1} . . b_{D-1}} X_{r, v_{1}}^{b_{1}}(v) . . X_{r, v_{D-1}}^{b_{D-1}}(v) \operatorname{tr}\left(\tau_{i} h_{e_{r}}(0,1 / 2) h_{\rho_{e_{r}}\left(X_{r}(v)\right)} \tau_{j} h_{\rho_{e_{r}}\left(X_{r}(v)\right)}^{-1} h_{e_{r}}(0,1 / 2)^{-1}\right)(3 .
\end{aligned}
$$

This time we will perform the $t, v$ integral to cancel the $\delta$-distribution. Thus we may as well perform the limits $\epsilon_{1}, \epsilon_{2} \rightarrow 0$ and cancel the $s$ and $r$ integrals first with the result that we are left with

$$
\begin{aligned}
& -\frac{1}{N} \int_{0}^{1 / 2} d t h_{e}(0, t) \frac{\tau_{j}}{2} h_{e}(t, 1 / 2) \int_{V} d^{D-1} v \delta(e(t), X(v)) \dot{e}^{a}(t) \times \\
& \times \epsilon_{a b_{1} . . b_{D-1}} X_{, v_{1}}^{b_{1}}(v) . . X_{, v_{D-1}}^{b_{D-1}}(v) \operatorname{tr}\left(\tau_{i} h_{e}(0,1 / 2) h_{\rho_{e}(X(v))} \tau_{j} h_{\rho_{e}(X(v))}^{-1} h_{e}(0,1 / 2)^{-1}\right)
\end{aligned}
$$

The integrand is supported at $t=1 / 2, v=0$ which is now a boundary point of the interval $[0,1 / 2]$ which is why the $\delta$ distribution picks up a factor of $1 / 2$ as compared to the analogous argumentation in [2.]. This leads to the result

$$
-\frac{1}{2 N} h_{e}(0,1 / 2) \frac{\tau_{j}}{2} \operatorname{tr}\left(\tau_{i} h_{e}(0,1 / 2) \tau_{j} h_{e}(0,1 / 2)^{-1}\right)=\frac{1}{4} \tau_{i} h_{e}(0,1 / 2)
$$

Putting things together and using $\delta g^{-1}=-g^{-1} \delta g g^{-1}$ we find

$$
\begin{aligned}
\left\{P_{i}^{e}, P_{j}^{e}\right\}_{\gamma}= & \int_{\Sigma} d^{3} x E_{k}^{a}(x)\left[-\frac{1}{N} \int_{V} d^{D-1} u \delta(x, X(u)) \epsilon_{a b_{1} . . b_{D-1}} X_{u_{1}}^{b_{1}}(u) . . X_{u_{D-1}}^{b_{D-1}}(u)\right] \times \\
\times & \quad \operatorname{tr}\left(\tau_{j}\left\{\frac{1}{4} \tau_{i} h_{e}(0,1 / 2)\right\} h_{\rho_{e}(X(u))} \tau_{k} h_{\rho_{e}(X(u))}^{-1} h_{e}(0,1 / 2)^{-1}\right) \\
& -\operatorname{tr}\left(\tau_{j} h_{e}(0,1 / 2) h_{\rho_{e}(X(u))} \tau_{k} h_{\rho_{e}(X(u))}^{-1} h_{e}(0,1 / 2)^{-1}\left\{\frac{1}{4} \tau_{i} h_{e}(0,1 / 2)\right\} h_{e}(0,1 / 2)^{-1}\right) \\
& -i \leftrightarrow j] \\
= & -\frac{1}{4 N} \int_{S_{e}}(* E)_{k}(x) \times \\
\times & {\left[\operatorname{tr}\left(\tau_{j} \tau_{i} h_{e}(0,1 / 2) h_{\rho_{e}(x)} \tau_{k} h_{\rho_{e}(x)}^{-1} h_{e}(0,1 / 2)^{-1}\right)\right.} \\
& -\operatorname{tr}\left(\tau_{j} h_{e}(0,1 / 2) h_{\rho_{e}(x)} \tau_{k} h_{\rho_{e}(x)}^{-1} h_{e}(0,1 / 2)^{-1} \tau_{i}\right) \\
& -i \leftrightarrow j] \\
= & -\frac{1}{4 N} \int_{S_{e}}\left[2 \operatorname{tr}\left(\left[\tau_{j}, \tau_{i}\right] h_{e}(0,1 / 2) h_{\rho_{e}(x)} * E(x) h_{\rho_{e}(x)}^{-1} h_{e}(0,1 / 2)^{-1}\right)\right] \\
= & \frac{f_{i j} k}{N} \int_{S_{e}} \operatorname{tr}\left(\tau_{k} h_{e}(0,1 / 2) h_{\rho_{e}(x)} * E(x) h_{\rho_{e}(x)}^{-1} h_{e}(0,1 / 2)^{-1}\right) \\
= & -f_{i j}{ }^{k} P_{k}^{e}
\end{aligned}
$$

as claimed.

Notice that the factor of $1 / 2$ that appeared in (3.43) is also required if the bracket $\{., .\}_{\gamma}$ is 
to satisfy the Leibniz rule : $\left\{P_{\gamma}^{e}, h_{e}\right\}_{\gamma}=\left\{P_{\gamma}^{e}, h_{e}(0,1 / 2)\right\} h_{e}(1 / 2,1)+h_{e}(0,1 / 2)\left\{P_{\gamma}^{e}, h_{e}(1 / 2,1)\right\}$ which is consistent with (3.25) if indeed $\left\{P_{\gamma}^{e}, h_{e}(0,1 / 2)\right\}=\tau_{i} h_{e}(0,1 / 2) / 4,\left\{P_{\gamma}^{e}, h_{e}(1 / 2,1)\right\}=$ $h_{e}(0,1 / 2)^{-1} \tau_{i} h_{e} / 4$.

Theorem 3.3 The bracket $\{., .\}_{\gamma}$ satisfies the Jacobi identity, moreover, it defines a nondegenerate two-form on $M_{\gamma}$, that is, it is a symplectic structure.

Proof of Theorem 3.3 :

i) Jacobi identity:

There are four kinds of double-brackets to check corresponding to $n$ momenta and $3-n$ holonomies appearing with $n=0,1,2,3$.

$n=0)$ :

This case is trivial

$$
\left\{\left(h_{e}\right)_{A B},\left\{\left(h_{e^{\prime}}\right)_{C D},\left(h_{e^{\prime \prime}}\right)_{E F}\right\}_{\gamma}\right\}_{\gamma}+\text { cyclic }=0
$$

since already the inner bracket vanishes by (3.24).

$n=1)$ :

Also this case is trivial

$$
\left\{\left(h_{e}\right)_{A B},\left\{\left(h_{e^{\prime}}\right)_{C D}, P_{i}^{e^{\prime \prime}}\right\}_{\gamma}\right\}_{\gamma}+\text { cyclic }=0
$$

because either the inner bracket already vanishes or the inner bracket gives a function depending only on holonomies by (3.25) and so the outer bracket is of the type (3.24) and vanishes. $n=2)$ :

This is the first non-trivial case and it is quite remarkable that the signs and numerical factors in (3.24)-(3.26) come in precisely the right way out of the regularized derivation of theorem 3.2

$$
\begin{aligned}
& \left\{h_{e},\left\{P_{i}^{e^{\prime}}, P_{j}^{e^{\prime \prime}}\right\}_{\gamma}\right\}_{\gamma}+\left\{P_{i}^{e^{\prime}},\left\{P_{j}^{e^{\prime \prime}}, h_{e}\right\}_{\gamma}\right\}_{\gamma}+\left\{P_{j}^{e^{\prime \prime}},\left\{h_{e}, P_{i}^{e^{\prime}}\right\}_{\gamma}\right\}_{\gamma} \\
= & -\delta^{e^{\prime} e^{\prime \prime}} f_{i j}{ }^{k}\left\{h_{e}, P_{k}^{e^{\prime}}\right\}_{\gamma}+\delta_{e}^{e^{\prime \prime}}\left\{P_{i}^{e^{\prime}}, \frac{\tau_{j}}{2} h_{e}\right\}_{\gamma}-\delta_{e}^{e^{\prime}}\left\{P_{j}^{e^{\prime \prime}}, \frac{\tau_{i}}{2} h_{e}\right\}_{\gamma} \\
= & \delta^{e^{\prime} e^{\prime \prime}} \delta_{e}^{e^{\prime}}\left(f_{i j}{ }^{k} \frac{\tau_{k}}{2} h_{e}+\frac{\tau_{j} \tau_{i}}{4} h_{e}-\frac{\tau_{i} \tau_{j}}{4} h_{e}\right) \\
= & \frac{1}{4} \delta^{e^{\prime} e^{\prime \prime}} \delta^{e^{\prime} e}\left(2 f_{i j}{ }^{k} \tau_{k}+\left[\tau_{j}, \tau_{i}\right]\right) h_{e}=0
\end{aligned}
$$

by definition of the structure constants.

$n=3$ :

This case is again easy, it just relies on the Jacobi identity for the generators of the Lie algebra of $G$ or, equivalently, for its structure constants :

$$
\left\{P_{i}^{e},\left\{P_{j}^{e^{\prime}}, P_{k}^{e^{\prime \prime}}\right\}_{\gamma}\right\}_{\gamma}+\text { cyclic }=\delta^{e^{\prime} e^{\prime \prime}} \delta_{e}^{e^{\prime}}\left(f_{j k}{ }^{l} f_{i l}{ }^{m}+\text { cyclic }\right) P_{m}^{e}=0
$$

ii) Non-degeneracy

Obviously, by (3.24)-(3.26) the symplectic structure $\Omega_{\gamma}$, if it exists, is diagonal with respect to the edge label,

$$
\Omega_{\gamma}=\sum_{e \in E(\gamma)} \Omega_{e}
$$

where each $\Omega_{e}$ is isomorphic with a Poisson structure $\Omega_{G}$ on the cotangent bundle $T^{*} G$ given by $\left\{h_{A B}, h_{C D}\right\}_{G}=0,\left\{P_{i}, h\right\}_{G}=\tau_{i} h / 2,\left\{P_{i}, P_{j}\right\}_{G}=-f_{i j}{ }^{k} P_{k}$. Thus in order to show regularity of $\Omega_{\gamma}$ it will be necessary to show regularity of $\Omega_{G}$, that is, $\Omega_{G}$ is not only a Poisson structure but actually a symplectic structure.

To see that $\Omega_{G}$ is everywhere nondegenerate on $G$ consider the atlas $\left(U_{\alpha}, \phi_{\alpha}\right)_{\alpha \in I}$ of $G$ given 
by open neighbourhoods $U_{\alpha}$ containing some point $h_{\alpha} \in G$ and charts defined by $\phi_{\alpha}^{-1}:=\exp$ : $V_{\alpha} \subset \mathbb{R}^{\operatorname{dim}(G)} \mapsto U_{\alpha} ;\left(\theta_{\alpha}^{j}\right) \mapsto \exp \left(\theta_{\alpha}^{j} \tau_{j} / 2\right) h_{\alpha}$. Obviously, the $\theta_{\alpha}^{j}$ serve as local coordinates of $G$. Let now $h \in G$ be given, then there exists $\alpha \in I,\left(\theta_{\alpha}^{j}\right) \in V_{\alpha}$ such that $h=\exp \left(\theta_{\alpha}^{j} \tau_{j} / 2\right) h_{\alpha}$. By choosing the size of the index set $I$ high enough we can assume that the range of each $\theta_{\alpha}^{j}$ is contained in an open interval containing zero as small as we wish. Let us now expand $h$ in powers of $\theta_{\alpha}^{j}$ in the relation $\left\{P_{j}, h\right\}_{G}=\tau_{j} h / 2$ then we find by comparing powers that $\left\{P_{j}, \theta^{k}\right\}=\delta_{j}^{k}+O(\theta)$ where $O(\theta)$ is a bounded function vanishing linearly in $\theta$. We conclude that the bracket when expressed in terms of the coordinates $P_{j}, \theta_{j}$ has locally the form of a block matrix consisting of four blocks of $\operatorname{dim}(G) \times \operatorname{dim}(G)$ matrices with the off-diagonal blocks given by plus/minus the identity matrix plus corrections of order $\theta_{\alpha}^{j}$ and the diagonal blocks consist of a zero matrix and of the matrix with $i j$-entries given by $f_{i j}{ }^{k} P_{k}$. This matrix is obviously invertible on $U_{\alpha}$ for any $\alpha$, proving our claim.

Finally we must show that $\Omega_{\gamma}$ is a surjection, that is, for any $\left(x_{e}, y_{e}\right)_{e \in E(\gamma)} \in \mathcal{E}_{\gamma}^{\prime}$ there exists $\left(x_{e}^{\prime}, y_{e}^{\prime}\right)_{e \in E(\gamma)} \in \mathcal{E}_{\gamma}$ such that $\left(x_{e}^{\prime}, y_{e}^{\prime}\right) \cdot \Omega_{e}=\left(x_{e}, y_{e}\right)$. Since $\mathcal{E}_{\gamma}$ is actually a Hilbert-manifold, we find $\left(\tilde{x}_{e}, \tilde{y}_{e}\right) \in \mathcal{E}_{\gamma}$ such that $\left(x_{e}, y_{e}\right)=\left(\tilde{x}_{e} \cdot \rho_{e}, \tilde{y}_{e} \cdot \sigma_{e}\right)$ thus the solution of our problem is given by $\left(x_{e}^{\prime}, y_{e}^{\prime}\right)=\left(\tilde{x}_{e} \cdot \rho_{e}, \tilde{y}_{e} \cdot \sigma_{e}\right) \cdot\left(\Omega_{e}\right)^{-1}\left(\Omega_{e}\right.$ interpreted as a matrix written pointwise in $\left.M_{\gamma}\right)$ provided we can show that this defines an element of $\mathcal{E}_{\gamma}$. This is somewhat non-trivial because $\Omega_{e}$ depends on $P_{i}^{e}$ which, a priori, can take arbitrarily large values. However, the normalizability of this vector follows from (3.18) which implies that in fact $P_{i}^{e}$ must be uniformly bounded in $e$.

In the appendix we show that $\Omega_{G}$ and therefore $\Omega_{\gamma}$ is even exact in the case of $G=U(1), S U(2)$ which one can probably prove also for general $G$ and we leave this as a future project.

\subsubsection{Continuum Phase Space from the Graph Phase Space}

In the previous subsection we established how the symplectic manifold $\left(M_{\gamma}, \Omega_{\gamma}\right)$ can be derived from the symplectic manifold $(M, \Omega)$ for every $\gamma \in \Gamma$. In this subsection we wish to show the opposite : in the limit that $\gamma$ grows ad infinitum in a prescribed way we find that $(M, \Omega)$ can be derived from $\left(M_{\gamma}, \Omega_{\gamma}\right)$. This lies at the heart of later constructions in which we use $\left(M_{\gamma}, \Omega_{\gamma}\right)$ as our starting point for quantization. Namely, as the formulation of the theory in terms of $M_{\gamma}$ is a certain kind of discretization, the result just stated means that the continuum limit exists and is the expected one on the classical level. On the other hand, the symplectic manifold $\left(M_{\gamma}, \Omega_{\gamma}\right)$ is straightforward to quantize on a Hilbert space $\left(\mathcal{H}_{\gamma},<., .>_{\gamma}\right)$ and the classical limit of this quantization is easily shown to give back $\left(M_{\gamma}, \Omega_{\gamma}\right)$. In other words, we can establish the chain of limits $\left(\mathcal{H}_{\gamma},<.,>_{\gamma}\right) \rightarrow_{\hbar \rightarrow 0}\left(M_{\gamma}, \Omega_{\gamma}\right) \rightarrow_{\gamma \rightarrow \infty}(M, \Omega)$ and the interesting question of the existence of the opposite limit will be subject of our companion paper [28].

In order to show that we can recover the continuum theory from the discrete one in the limit of infinite graphs we consider a certain one-parameter family of cubic lattices $\epsilon \mapsto \gamma_{\epsilon}$ where $\epsilon$ is associated with the length of the edges or links of the graph with respect to a certain background metric and the limit $\epsilon \rightarrow 0$ corresponds to sending the graph to the continuum $\Sigma$. More precisely, we have the following :

The manifold $\Sigma$ is described by an atlas of charts $\left(U_{\iota}, X_{\iota}\right)_{\iota \in \mathcal{I}}$ where $U_{\iota}$ is an open region in $\Sigma$ and $X_{\iota}^{a}: V_{\iota} \subset \mathbb{R}^{D} \mapsto U_{\iota},\left(t^{1}, . ., t^{D}\right) \mapsto X_{\iota}(t)$ is a local trivialization of $U_{\iota}$, that is, a smooth orientation preserving diffeomorphism. Consider an arbitrary but fixed decomposition $\mathcal{R}$ of $\Sigma$ into mutually disjoint, except for common boundary points, compact regions $R$ which is fine enough such that every $R$ lies in the domain of a chart and choose $\iota(R) \in \mathcal{I}$ to be such that $R \in U_{\iota(R)}$ (at this point we do not even need the cover $\left\{U_{i}\right\}$ to be locally finite or $\Sigma$ to be paracompact although this is an assumption which goes into the definition of $\left.\Gamma_{\sigma}^{\omega}\right)$. Without loss of generality we can assume that each $R$ is diffeomorphic to a polyhedron of $\mathbb{R}^{D}$ and we 
fix for each of its faces an orientation. Also, if $\Sigma$ is not compact we take a refinement of the atlas if necessary such that each $R$ has finite Lebesgue measure.

Now $R$ is the image under $X_{R}:=X_{\iota(R)}$ of a compact region $V_{R}$, in fact a polyhedron, in $\mathbb{R}^{D}$ which can always be decomposed, for sufficiently small $\epsilon$, into regular cubes of volume $\epsilon^{D}$ with respect to the Euclidean metric of $\mathbb{R}^{D}$ possibly up to a subset near the the boundary of $X_{R}^{-1}(R)$. Let then $C_{R}$ be the union of those cubes which fit into $V_{R}$.

Now each region $C_{R}$ is filled exactly with cubes of volume $\epsilon^{D}$ and these cubes define a regular, oriented cubic lattice $\gamma_{R}^{0}$ in $C_{R}$, the orientation of the edges is chosen to be such that each of them points in the positive coordinate axis direction of $\mathbb{R}^{D}$. Let $P_{\gamma_{R}^{0}}^{0}$ be the dual decomposition of $V_{R}$ obtained as follows : choose for each edge $e_{R}^{0}$ of $\gamma_{R}^{0}$ the open face $S_{e_{R}^{0}}^{0}$ to be the regular cubic hyperplane orthogonal to $e_{R}^{0}$ of area $\epsilon^{D-1}$ which cuts $e_{R}^{0}$ in the middle, is pierced by $e_{R}^{0}$ in its center and carries the orientation defined by choosing the tangent direction of $e_{R}^{0}$ to be the direction of its unit normal vector. The collection of all these faces $S_{e_{R}^{0}}^{0}$ can be completed uniquely to the unique, minimal cubic polyhedronal complex $C_{R}^{\prime}$ which contains all of them. We assume w.l.g. that $C_{R}^{\prime}$ fits into $V_{R}$ (decrease the size of $C_{R}$ by deleting some cubes on its boundary if necessary). Notice that $C_{R}^{\prime}$ necessarily covers $C_{R}$. We complete the polyhedronal decomposition of $V_{R}$ by choosing $X_{R}^{-1}(R)-C_{R}^{\prime}$ as the final polyhedron to cover $V_{R}$. Since the boundary of $V_{R}$ is already oriented this obviously defines an oriented polyhedronal decomposition of $X_{R}^{-1}(R)$ dual to $\gamma_{R}^{0}$.

Finally, we consider the images $\gamma_{R}=X_{\iota(R)}\left(\gamma_{R}^{0}\right), S_{e}=X_{\iota(R)}\left(S_{e_{R}^{0}}^{0}\right) P_{\gamma_{R}}=X_{\iota(R)}\left(P_{\gamma_{R}^{0}}^{0}\right)$ and the corresponding variables $h_{e}^{R}, P_{i}^{e, R}$ for each $R \in \mathcal{R}$. The unions $\gamma_{\epsilon}:=\cup_{R \in \mathcal{R}} \gamma_{R}$ and $P_{\gamma_{\epsilon}}:=$ $\cup_{R \in \mathcal{R}} P_{\gamma_{R}}^{0}$ define an oriented graph in $\Sigma$ and an oriented decomposition of $\Sigma$. It is not yet a decomposition dual to $\gamma$ since it is not minimal : it becomes minimal if we remove all the boundaries $\partial R$. Let the resulting dual decomposition also be denoted by $P_{\gamma_{\epsilon}}$.

Notice that the region $\Sigma-\left[\cup_{R \in \mathcal{R}} X_{R}\left(C_{R}^{\prime}\right)\right]$ does not contain any piece of $\gamma_{\epsilon}$, however, its Lebesgue measure vanishes in the limit $\epsilon \rightarrow 0$ because it tends to $\cup_{R} \partial R$. We could avoid this by adding edges to $\gamma$ connecting the $\gamma_{R}$ which are contained in neigbouring $R$ but the resulting lattice may not be of cubic topology any longer. Since we will not need those edges for the sake of our argument, we will leave things as they are.

Let us fix a specific $R$ and define $v_{R}:=X_{R}\left(v_{R}^{0}\right), \quad e_{R I}(v):=X_{R}\left(e_{R I}^{0}\left(v^{0}\right)\right), \quad S_{R}^{I}(v):=$ $X_{R}\left(S_{e_{R I}^{0}\left(v_{R}^{0}\right)}^{0}\right)$ respectively to be the image under $X^{R}$ of a vertex, edge and face of $\gamma_{R}^{0}$ respectively. Here $e_{R I}^{0}\left(v_{R}^{0}\right)$ denotes the straight line into the positive $I$-direction between the vertices $v_{R}^{0}$ and $v_{R}^{0}+\epsilon b_{I}$ where $\left\{b_{I}\right\}_{I=1}^{D}$ denotes the standard oriented orthonormal basis of $\mathbb{R}^{D}$ (sometimes $v_{R}^{0}+\epsilon b_{I}$ is not a vertex of $\gamma_{R}^{0}$ in which case we set $e_{R I}^{0}\left(v_{R}^{0}\right)=v_{R}^{0}=S_{e_{R I}^{0}\left(v_{R}^{0}\right)}^{0}$. Consider also for any $x \in R$ the vector fields $Y_{R I}^{a}(x):=X_{R, I}^{a}(t)_{x=X_{R}(t)}$ and co-vector densities of weight minus one $n_{R a}^{I}(x):=\frac{1}{(D-1) !} \epsilon_{a b_{1} . . b_{D-1}} \epsilon^{I J_{1} \ldots J_{D-1}} Y_{R J_{1}}^{b_{1}}(x) . . Y_{R J_{D-1}}^{b_{D-1}}(x)$. Since $X_{R}: V_{R} \mapsto R$ is an orientation preserving diffeomorphism it is clear that $\operatorname{det}((Y))=n_{a}^{I} Y_{I}^{a}>0$ everywhere in $R$.

We define now as in (3.15) for every vertex $v$ of $\gamma_{R}$ the functions (we drop the label $R$ )

$$
\begin{aligned}
h_{I}(v) & :=h_{e_{I}(v)}(A) \\
P_{i}^{I}(v) & :=-\frac{1}{N} \operatorname{tr}\left(\tau_{i} h_{e_{I}(v)}(0,1 / 2)\left[\int_{S^{I}(v)} h_{\rho_{e_{I}(v)}(x)} * E(x) h_{\rho_{e_{I}(v)}(x)}^{-1}\right] h_{e_{I}(v)}(0,1 / 2)^{-1}\right)(A, E)
\end{aligned}
$$

which defines a map $D_{\epsilon}: M \mapsto M_{\gamma}$.

On the other hand, consider now the following functions on $M_{\gamma}\left(v\right.$ is again a vertex of $\gamma_{R}$ and we drop the label $R$ )

$$
\begin{aligned}
A_{a}^{(\epsilon) i}(v) & :=-2 \frac{n_{a}^{I}}{\operatorname{det}(Y) N \epsilon} \operatorname{tr}\left(\tau_{i} h_{I}(v)\right) \\
E_{i}^{(\epsilon) a}(v) & :=\frac{Y_{I}^{a}}{\operatorname{det}(Y) \epsilon^{D-1}} P_{i}^{I}(v)
\end{aligned}
$$


Suppose first that $\left(h_{I}(v), P_{i}^{I}(v)\right) \in G \times \operatorname{Lie}(G)$ are obtained via the map $D_{\epsilon}$. Then, using the smoothness of the fields $(A, E)$ it is easy to see that $A_{a}^{(\epsilon) i}(v)-A_{a}^{i}(v)$ and $E_{i}^{(\epsilon) a}(v)-E_{i}^{a}(v)$ are both of order $\epsilon$.

We now select from the one-parameter family of lattices $\gamma_{\epsilon}$ and decompositions $P_{\gamma_{\epsilon}}$ a sequence of lattices $\gamma_{n}$ with the property that $\gamma_{n+1}$ and $P_{\gamma_{n+1}}$ respectively are refinements of $\gamma_{n}$ and $P_{\gamma_{n}}$ respectively. It is easy to see that the following sequence does the job : Start with some $\epsilon_{0}>0$ and call $\gamma_{0}:=\gamma_{\epsilon_{0}}, P_{0}:=P_{\gamma_{\epsilon_{0}}}$ respectively. Now consider the sequence $\epsilon_{n}:=\epsilon_{0} / 3^{n}$. The corresponding $\gamma_{n}:=\gamma_{\epsilon_{n}}, P_{n}:=P_{\gamma_{\epsilon_{n}}}$ are obtained iteratively as follows :

Given $\gamma_{R n}^{0}$, subdivide each of the cubes it defines into $3^{D}$ axis parallel cubes of equal volume $\left(\epsilon_{n} / 3\right)^{D}$ and similarly for $P_{\gamma_{R n}}^{0}$. Both of these lattices obviously refine the previous ones respectively.

To see that the refinement of $P_{\gamma_{R n}^{0}}^{0}$ has all the properties that we required it to have in the above construction of a decomposition of $V_{R}$ dual to the refinement of $\gamma_{R n}^{0}$ we remark the following :

If we consider $\gamma_{R n}^{0}$ as a sublattice of an infinite regular cubic lattice $L_{n}$ in $\mathbb{R}^{D}$ then $P_{\gamma_{R n}^{0}}^{0}$ satisfies the required proprties if and only if its restriction to $C_{R}^{\prime}$ is defined by a sublattice of the lattice $L_{n}^{\prime}$ obtained from $L_{n}$ by translating it by the vector $\epsilon_{n} \sum_{I=1}^{D} b_{I} / 2$. In other words, if we label the vertices of $L_{n}$ by the n-tuples $\left(\epsilon_{n} n_{I}\right)_{I=1}^{D}, n_{I} \in Z$ then the vertices of $L_{n}^{\prime}$ are labelled by the ntuples $\left(\epsilon_{n}\left[n_{I}^{\prime}+1 / 2\right]\right)_{I=1}^{D}, n_{I}^{\prime} \in Z$. Now the points of the refinements of $L_{n}$ and $L_{n}^{\prime}$ respectively are labelled by $\left(\epsilon_{n} n_{I} / 3\right)_{I}=\epsilon_{n+1} n_{I}$ and $\left(\epsilon_{n}\left[n_{I}^{\prime} / 3+1 / 2\right]\right)_{I}=\left(\epsilon_{n}\left[\left(n_{I}^{\prime}+1\right) / 3+1 / 6\right]\right)_{I}=\left(\epsilon_{n+1}\left[n_{I}^{\prime \prime}+1 / 2\right]\right)_{I}$, in other words, the refinements coincide with $L_{n+1}$ and $L_{n+1}^{\prime}$ respectively. Notice that our procedure would work also if we would choose to refine by $k$ instead of 3 where $k>3$ can be any odd integer.

To complete the decomposition we add cubes to these refinements as to fill $V_{R}$ as densely as possible according to the rules we specified above (also deleting cubes if necessary as discussed above) and thus obtain, after mapping with $X_{R}, \gamma_{n+1}$ and $P_{\gamma_{n+1}}$.

We remark without proof that only cubic lattices seem to have the property that there are refinements such that a dual decomposition of the refinement can be a refinement of the dual decomposition (consider a simplicial decomposition to see the arising problems).

Now that we know that if $v$ is a vertex of $\gamma_{n}$ for some $n$ then it is a vertex of all $\gamma_{m}$ for all $m \geq n$, the limit $\epsilon \rightarrow 0$ is well defined and we have, provided that $A_{a}^{\left(\epsilon_{n}\right) i}(v)=: A_{a}^{(n) i}(v), E_{i}^{\left(\epsilon_{n}\right) a}(v)=$ : $E_{i}^{(n) a}(v)$ are defined via $D_{n}:=D_{\epsilon_{n}}$

$$
\begin{aligned}
& \lim _{\epsilon \rightarrow 0}\left[A_{a}^{(\epsilon) i}(v)-A_{a}^{i}(v)\right]:=\lim _{n \rightarrow \infty}\left[A_{a}^{(n) i}(v)-A_{a}^{i}(v)\right] \\
& \lim _{\epsilon \rightarrow 0}\left[E_{i}^{(\epsilon) a}(v)-E_{i}^{a}(v)\right]:=\lim _{n \rightarrow \infty}\left[E_{a}^{(n) a}(v)-E_{i}^{a}(v)\right]
\end{aligned}
$$

where convergence is pointwise on $M$. In other words, the map $D_{n}: M \mapsto M_{n} \subset M_{\gamma_{n}}$ is invertible in the limit $n \rightarrow \infty$. To see that also the symplectic structure $\Omega$ of $M$ is recovered in this limit we notice first that for each $f_{a}^{i}, F_{i}^{a} \in \mathcal{S}$ we have $F(A)=\lim _{n \rightarrow \infty} F \cdot A^{(n)}(A), E(f)=$ $\lim _{n \rightarrow \infty} E^{(n)}(A, E) \cdot f$ pointwise in $M$ where

$$
\begin{aligned}
F \cdot A^{(n)} & :=\sum_{v \in V\left(\gamma_{n}\right)} \epsilon_{n}^{D}(\operatorname{det}(Y))(v) F_{a}^{i}(v) A_{a}^{(n) i}(v) \\
E^{(n)} \cdot f & :=\sum_{v \in V\left(\gamma_{n}\right)} \epsilon_{n}^{D}(\operatorname{det}(Y))(v) E_{i}^{(n) a}(v) f_{a}^{i}(v)
\end{aligned}
$$


Theorem 3.4 The bracket defined by

$$
\begin{aligned}
\left\{F(A), F^{\prime}(A)\right\}^{\prime} & :=\lim _{n \rightarrow \infty}\left\{F \cdot A^{(n)}, F^{\prime} \cdot A^{(n)}\right\}_{\gamma_{n}} \\
\{E(f), F(A)\}^{\prime} & :=\lim _{n \rightarrow \infty}\left\{E^{(n)} \cdot f, F \cdot A^{(n)}\right\}_{\gamma_{n}} \\
\left\{E(f), E\left(f^{\prime}\right)\right\}^{\prime} & :=\lim _{n \rightarrow \infty}\left\{E^{(n)} \cdot f, E^{(n)} \cdot f^{\prime}\right\}_{\gamma_{n}}
\end{aligned}
$$

for all $f_{a}^{i}, F_{i}^{a}, f_{a}^{\prime i}, F_{i}^{\prime a} \in \mathcal{S}$ coincides with the symplectic structure $\Omega$ on $M$ defined by (3.10).

Proof of Theorem 3.4 :

We simply have to use the symplectic structure of $\left(M_{\gamma_{n}}, \Omega_{\gamma_{n}}\right)$ and take the limit using (3.51). In the notation of this subsection, the symplectic structure labelled by $\gamma_{n}$ can be written

$$
\begin{aligned}
& \left\{\left(h_{I}(v)\right)_{A B},\left(h_{J}\left(v^{\prime}\right)\right)_{C D}\right\}_{\gamma_{n}}=0 \\
& \left\{P_{i}^{I}(v), h_{J}\left(v^{\prime}\right)\right\}_{\gamma_{n}}=\delta_{J}^{I} \delta_{v, v^{\prime}} \frac{\tau_{i}}{2} h_{J}(v) \\
& \left\{P_{i}^{I}(v), P_{j}^{J}\left(v^{\prime}\right)\right\}_{\gamma_{n}}=-\delta^{I J} \delta_{v v^{\prime}} f_{i j}{ }^{k} P_{k}^{I}(v)
\end{aligned}
$$

[1.]

Then (3.53) is obvious since the right hand side vanishes already at any finite $n$.

[2.]

We have at fixed $n$ for the right hand side of (3.54)

$$
\begin{aligned}
& \left\{E^{(n)} \cdot f, F \cdot A^{(n)}\right\}_{\gamma_{n}}=\sum_{v, v^{\prime} \in V\left(\gamma_{n}\right)} \epsilon_{n}^{2 D}(\operatorname{det}(Y))(v)(\operatorname{det}(Y))\left(v^{\prime}\right) f_{a}^{i}(v) F_{j}^{b}\left(v^{\prime}\right) \times \\
& \times\left[-2 \frac{n_{b}^{J}\left(v^{\prime}\right)}{\operatorname{det}(Y)\left(v^{\prime}\right) N \epsilon_{n}} \frac{Y_{I}^{a}(v)}{\operatorname{det}(Y)(v) \epsilon_{n}^{D-1}}\right]\left\{P_{i}^{I}(v), \operatorname{tr}\left(\tau_{j} h_{J}\left(v^{\prime}\right)\right)\right\}_{\gamma_{n}} \\
= & -\frac{2}{N} \sum_{v \in V\left(\gamma_{n}\right)} \epsilon_{n}^{D} f_{a}^{i}(v) F_{j}^{b}(v)\left[\sum_{I}\left(n_{b}^{I} Y_{I}^{a}\right)(v) \operatorname{tr}\left(\tau_{j} \frac{\tau_{i}}{2} h_{I}(v)\right)\right] \\
= & -\frac{2}{N} \sum_{v \in V\left(\gamma_{n}\right)} \epsilon_{n}^{D} f_{a}^{i}(v) F_{j}^{b}(v)\left[-\frac{N}{2} \delta_{i j} \delta_{b}^{a} \operatorname{det}(Y)(v)+\sum_{I}\left(n_{b}^{I} Y_{I}^{a}\right)(v) \operatorname{tr}\left(\tau_{j} \frac{\tau_{i}}{2}\left[h_{I}(v)-1\right]\right)\right] \\
= & \left\{\sum_{v \in V\left(\gamma_{n}\right)} \epsilon_{n}^{D}\left[\operatorname{det}(Y) f_{a}^{i} F_{i}^{a}\right](v)\right\} \\
& -\frac{2}{N}\left\{\sum_{v \in V\left(\gamma_{n}\right)} \epsilon^{D}\left[f_{a}^{i} F_{j}^{b}\right](v) \sum_{I}\left(n_{b}^{I} Y_{I}^{a}\right)(v) \operatorname{tr}\left(\tau_{j} \frac{\tau_{i}}{2}\left[h_{I}(v)-1\right]\right)\right\}
\end{aligned}
$$

Consider the first term in the last equality of (3.57). We can write it as

$$
\sum_{R \in \mathcal{R}} \sum_{v^{0} \in \gamma_{R}^{0}} \epsilon_{n}^{D} \mid \operatorname{det}\left(\left(\frac{\partial X}{\partial t}_{t\left(v^{0}\right)} \mid\left[f_{a}^{i} F_{i}^{a}\right]\left(X\left(t\left(v^{0}\right)\right)\right)\right.\right.
$$

which defines a Riemann sum for the expression

$$
\sum_{R \in \mathcal{R}} \int_{C_{R}} d^{D} t \mid\left(\operatorname { d e t } \left(\left(\frac{\partial X}{\partial t}\right)_{\mid t} \mid\left[f_{a}^{i} F_{i}^{a}\right](X(t))=F(f)-\sum_{R \in \mathcal{R}} \int_{R-X_{R}\left(C_{R}\right)} d^{D} x\left[f_{a}^{i} F_{i}^{a}\right](x)\right.\right.
$$

and the second integral in (3.59) vanishes in the limit $n \rightarrow \infty$ (that is $C_{R} \rightarrow R$ ). To see this, notice that in the limit $n \rightarrow \infty$ the integral $\int_{R-X_{R}\left(C_{R}\right)} d^{D} x\left[f_{a}^{i} F_{i}^{a}\right](x)$ becomes $\int_{\partial R} d^{D} x\left[f_{a}^{i} F_{i}^{a}\right](x)$ which vanishes for non-distributional spaces of test functions.

Turning to the second term in (3.57) we notice that there exists a positive constant $k$ such that $\left.\mid \operatorname{tr}\left(\tau_{j} \frac{\tau_{i}}{2}\left[h_{I}(v)-1\right]\right)\right] \mid \leq k \epsilon_{n}$ as $n \rightarrow \infty$ independent of the indices and $v$ because $A$ is a smooth and bounded function. Thus we see that the second term vanishes in the limit $n \rightarrow \infty$. 
[3.]

Finally, at fixed $n$ the right hand side of (3.55) becomes

$$
\begin{aligned}
\left\{E^{(n)} \cdot f, E^{(n)} \cdot f^{\prime}\right\}_{\gamma_{n}} & =\sum_{v, v^{\prime} \in V\left(\gamma_{n}\right)} \epsilon_{n}^{2}\left[f_{a}^{i} Y_{I}^{a}\right](v)\left[f_{b}^{j \prime} Y_{J}^{b}\right]\left(v^{\prime}\right)\left\{P_{i}^{I}(v), P_{j}^{J}\left(v^{\prime}\right)\right\}_{\gamma_{n}} \\
& =-f_{i j}{ }^{k} \sum_{v \in V\left(\gamma_{n}\right)} \epsilon_{n}^{2}\left[\sum_{I}\left(f_{a}^{i} Y_{I}^{a} f_{b}^{j \prime} Y_{I}^{b} P_{k}^{I}\right)(v)\right] \\
& =-f_{i j}{ }^{k} \epsilon_{n} \sum_{v \in V\left(\gamma_{n}\right)} \epsilon_{n}^{D}\left[\sum_{I}\left(f_{a}^{i} Y_{I}^{a} f_{b}^{j \prime} Y_{I}^{b}\left[E_{i}^{c} n_{c}^{I}+\left\{\frac{P_{k}^{I}}{\epsilon_{n}^{D-1}}-E_{i}^{c} n_{c}^{I}\right\}\right]\right)(v)\right]
\end{aligned}
$$

The whole sum is just an approximation for a Riemann integral times $\epsilon_{n}$. The term in the curly bracket approaches zero as $n \rightarrow 0$ and is therefore, together with the first term in the square bracket, integrable against the product of test functions displayed. Thus, the whole expression vanishes in the limit $n \rightarrow \infty$.

\subsection{Structured Graphs as Labels for Generalized Projective Families}

A natural question to ask is whether one can identify $(M, \Omega)$ with (the limit of) a generalized projective sequence of symplectic manifolds $\left(M_{\gamma}, \Omega_{\gamma}\right)$. The answer is affirmative but somewhat involved because we first must introduce new labels for projective families :

First of all, the family $\left(M_{\gamma}, \Omega_{\gamma}\right)$ does not only depend on the graph $\gamma$ but actually on the set $\mathcal{L}$ of structured graphs $l=\left(\gamma, P_{\gamma}, \Pi_{\gamma}\right)$ consisting of a graph $\gamma$, a polyhedronal decomposition $P_{\gamma}$ dual to it and a choice of paths $\rho_{e}(x) \in \Pi_{\gamma}$ adapted to $\gamma, P_{\gamma}$ where $\rho_{e}(x) \subset S_{e}, e \in E(\gamma), x \in S_{e}$. The family $\mathcal{L}$ is partially ordered by inclusion but it is in general wrong that given two elements $l, l^{\prime} \in \mathcal{L}$ there exists a common refinement, that is, an element $\tilde{l} \in \mathcal{L}$ such that $l, l^{\prime} \subset \tilde{l}$. In other words, the inclusion relation does not equip $\mathcal{L}$ with the structure of a directed set on which the structure of a generalized projective limit crucially depends.

In order to proceed, we therefore must first modify the partial order. To motivate our choice we begin with the following observation :

Given a graph $\gamma$ the second and third entry of a structured graph $l$ such that $\gamma(l)=\gamma$ are largely arbitrary. On the other hand, if we consider structured graphs $l, l^{\prime}$ with $\gamma(l)=\gamma\left(l^{\prime}\right)$ then by construction we can always find a diffeomorphism that preserves $\gamma(l)$ and maps $P_{\gamma}, \Pi_{\gamma}$ to $P_{\gamma^{\prime}}, \Pi_{\gamma^{\prime}}$. This follows from the fact that all the $S_{e}, \rho_{e}(x) \subset S_{e}, x \in S_{e}$ are obtained via a diffeomorphism from a universal object by definition 3.5. Now, while the actual values of the $P^{e}$ that we construct from $l$ or $l^{\prime}$ respectively may differ (the $h_{e}$ are evidently the same), the Poisson algebras, that is to say the algebra of Hamiltonian vector fields, that we obtain are identical. Moreover, let us consider the $h_{e}$ as elements of the space of smooth functions $C^{\infty}\left(\mathcal{C}_{\gamma}\right)$ of the configuration space $\mathcal{C}_{\gamma}$ of $M_{\gamma}$ (in fact they are coordinate functions) and the $P^{e}$ as elements of the space of vector fields $V\left(\mathcal{C}_{\gamma}\right)$ on $\mathcal{C}_{\gamma}$ via the map $\left(h_{e}, P_{j}^{e}\right) \mapsto$ $\left(h_{e}, \operatorname{tr}\left(\left(\tau_{j} h_{e}\right)^{T} \partial / \partial h_{e}\right)\right.$. The space $C^{\infty}\left(\mathcal{C}_{\gamma}\right) \times V\left(\mathcal{C}_{\gamma}\right)$ is equipped with the Lie algebra structure $\left[(f, u),\left(f^{\prime}, u^{\prime}\right)\right]=\left(u\left(f^{\prime}\right)-u^{\prime}(f),\left[u, u^{\prime}\right]\right)$ which is evidently closed and isomorphic with the Poisson bracket structure as obtained from both $l, l^{\prime}$. In this form it is particularly obvious that both $l, l^{\prime}$ give rise to the same Lie algebra.

What this means is that the information contained in $l$ beyond that of $\gamma(l)$ is irrelevant as far as the Poisson algebra is concerned. Since it is the Poisson structure which sets the correspondence with quantum theory we will obtain isomorphic quantum theories from both $l, l^{\prime}$. The additional information contained in $l$ however comes in when we consider the classical limit of the theory. Namely, the coherent states constructed in [42, 43, 44] are sensitive to the size and shape of the $S_{e}$ as well as the precise choice of the paths $\rho_{e}$. 
These considerations shed light on the question why we have largely abused notation when writing $\gamma$ instead of $l$ and is also reflected in the subsequent definition.

Definition 3.7 We say that $\left(\gamma, P_{\gamma}, \Pi_{\gamma}\right) \prec\left(\gamma^{\prime}, P_{\gamma^{\prime}}, \Pi_{\gamma^{\prime}}\right)$ provided that $\gamma(l) \subset \gamma\left(l^{\prime}\right)$ and that $l, l^{\prime}$ are equivalent, $l \equiv l^{\prime}$, if $\gamma(l)=\gamma\left(l^{\prime}\right)$.

In other words, there are no conditions at all on the second and third entry of a structured graph. In particular, we identify $l$ with $l^{\prime}$ if one obtains $l^{\prime}$ form $l$ by applying a diffemorphism that preserves $\gamma(l)$. The subsequent two lemmas are then almost trivial.

Lemma 3.2 The relation $\prec$ defined in definition 3.7 defines a partial order.

Proof of Lemma 3.2 :

1) Reflexivity : $l \prec l$ since $\gamma(l)=\gamma(l)$.

2) Antisymmetry : $l \prec l^{\prime}$ and $l^{\prime} \prec l$ implies $\gamma(l)=\gamma\left(l^{\prime}\right)$, that is, $l \equiv l^{\prime}$.

3) Transitivity : $l \prec l^{\prime}$ and $l^{\prime} \prec l^{\prime \prime}$ implies $\gamma(l) \subset \gamma\left(l^{\prime}\right) \subset \gamma\left(l^{\prime \prime}\right)$, thus $l \prec l^{\prime \prime}$.

Lemma 3.3 The set $\mathcal{L}$ is directed, that is, for any given $l, l^{\prime} \in \mathcal{L}$ there exists $\tilde{l} \in \mathcal{L}$ such that $l, l^{\prime} \prec \tilde{l}$. Such an element $\tilde{l}$ is called a common refinement of $l, l^{\prime}$.

Proof of Lemma 3.3 :

Given $l, l^{\prime} \in \mathcal{L}$ consider the graph $\tilde{\gamma}:=\gamma(l) \cup \gamma\left(l^{\prime}\right)$. Choose any $\tilde{l} \in \mathcal{L}$ such that $\tilde{\gamma}=\gamma(\tilde{l})$. Then $l, l^{\prime} \prec \tilde{l}$.

Next we need the notion of a projection of symplectic manifolds.

Definition 3.8 Let $l \prec l^{\prime}$, consider any edge $e \in E(\gamma)$ and find the edges $e_{1}^{\prime}, . ., e_{n}^{\prime} \in E\left(\gamma^{\prime}\right)$ such that $e=e_{1}^{\prime} \circ . . \circ e_{n}^{\prime}$. We then define the following projection

$$
p_{l^{\prime} l}: M_{l^{\prime}} \mapsto M_{l} ; h_{e}:=h_{e_{1}^{\prime}} . . h_{e_{n}^{\prime}} \text { and } P^{e}:=P^{e_{1}^{\prime}}
$$

It is obvious that $p_{l^{\prime} l}$ is onto for $l \prec l^{\prime}$ except in the presence of boundary conditions in which case the $P_{i}^{e}$ for sufficiently small $S^{e}$ would be bounded. As a map between the $\bar{M}_{l}$ it would be onto. Define $m_{l}=\left\{h_{e}, P^{e}\right\}_{e \in E(\gamma)}$ and consider an array of non-singular $\operatorname{dim}(G) \times \operatorname{dim}(G)$ matrices $\lambda=\left\{\lambda_{e}\right\}$ with an action on the points of $M_{l}$ given by $\lambda \cdot m_{l}=\left\{h_{e}, \lambda_{e} P^{e}\right\}$.

Definition 3.9 i) Consider the uncountable direct product $\mathcal{M}:=\times_{l \in \mathcal{L}} M_{l}$, then the following subset

$$
\mathcal{M}_{\infty}:=\left\{\left(m_{l}\right)_{l \in \mathcal{L}} \in \mathcal{M} ; \exists \lambda^{l l^{\prime}} \ni \lambda^{l l^{\prime}} \cdot m_{l}=p_{l^{\prime} l}\left(m_{l^{\prime}}\right) \forall l \prec l^{\prime}\right\}
$$

is called a generalized projective limit of the $M_{l}$.

ii) A family of symplectic structures $\left(\Omega_{l}\right)_{l \in \mathcal{L}}$ is called a self-consistent or generalized projective family provided that the associated Poisson brackets project in the usual way

$$
p_{l^{\prime} l}^{*}\{f, g\}_{l}:=\left\{p_{l^{\prime} l}^{*} f, p_{l^{\prime} l}^{*} g\right\}_{l^{\prime}} \forall f, g \in C^{\infty}\left(M_{l}\right)
$$

that is, the $p_{l^{\prime} l}$ are "non-invertible" symplectomorphisms.

It is easy to see that the symplectic structures $\Omega_{l}$ (or $\Omega_{\gamma}$ as we called them all the time) that we defined in section 3.2 .3 form indeed a self-consistent family of symplectic structures on $M_{l}$ (or $M_{\gamma}$ ). This follows, as already said, from the astonishing fact that the $\Omega_{\gamma}$ are completely insensitive to the size and shape of the faces of $P_{\gamma}$ and the choice of the paths of $\Pi_{\gamma}$ as long as all the requirements of a dual decomposition are met. This is precisely the contents of the identities (3.24) - (3.26). This observation is tied to the fact that the smearing functions, edges 
and faces, are sufficiently singular and that the smearing process is background metric independent, so that only topological characteristics, such as intersection numbers of edges with faces, are the results of the calculation and are thus completely shape independent, they are locally diffeomorphism invariant (i.e. invariant under locally non-trivial diffeomorphisms). Once more, this observation is also the logic behind definition 3.7 and behind labelling $\left(M_{\gamma}, \Omega_{\gamma}\right)$ only by elements of $\Gamma$ rather than by elements of $\mathcal{L}$.

Let us then summarize :

We have shown in this subsection that the family of differentiable manifolds $\left(M_{l}\right)$ can be given the structure of a generalized projective limit $\mathcal{M}_{\infty}$ and the family of symplectic structures thereon can be given the structure of a self-consistent family of symplectic structures.

In subsection 3.2 .3 on the other hand we showed that there is a sequence $l_{n} \in \mathcal{L}$ (there denoted $\gamma_{n}$ ) such that $M=\lim _{n \rightarrow \infty} M_{l_{n}}$ and $\Omega=\lim _{n \rightarrow \infty} \Omega_{l_{n}}$ (pointwise limits). Moreover, $l_{m} \prec l_{n}$ for all $m \leq n$, so the sequence is linearly ordered. The points $m_{l_{n}}$ defined in section 3.2.3 belong to $M_{l_{n}}$. By construction, we can extend every such sequence $m_{l_{n}}$ to a sequence $\left(m_{l}\right)_{l \in \mathcal{L}} \in \mathcal{M}_{\infty}$. (Explicitly, the array of matrices is for $n>m \rightarrow \infty$ approximately given by $\left.\lambda_{e i j}^{l_{m} l_{n}}=\delta_{i j}\left(\epsilon_{n} / \epsilon_{m}\right)^{D-1}\right)$. It follows that the sequence $\left(m_{l_{n}}\right)$ can be embedded into a generalized projective sequence which in turn defines a point of $\mathcal{M}_{\infty}$. Likewise, the standard symplectic manifold $(M, \Omega)$ can be identified with the sequence $\left(M_{l_{n}}, \Omega_{l_{n}}\right)$ of symplectic manifolds which in turn can be extended to a subset of the generalized projective limit and self-consistent symplectic structures thereon respectively (with respect to the generalized projective limit $\mathcal{M}_{\infty}$ ).

Remark :

Of course, we have displayed $(M, \Omega)$ only as the union of a very special subset of all generalized projective sequences. An arbitrary generalized projective sequence wil not have any obvious interpretation in terms of connections and electric fields on any smooth manifold $\Sigma$. This is possible because the set of graphs in - and dual decompositions of $\Sigma$ have much more structure than the set of points of $\Sigma$. In fact, the picture that emerges is completely combinatorical and only very special configurations of graphs and dual decomposition allow a manifold interpretation. In a sense, without specifying the embedding of abstract graphs and dual decompositions into a concrete $\Sigma$ we are treating all manifolds $\Sigma$ simultaneously. Thus, although in the canonical approach to quantum gravity one starts with a given differential manifold, the emerging classical and quantum theory does not depend any longer on the particular choice of $\Sigma$. Only if one insists on a manifold interpretation there will be restrictions on possible graphs (they have to agree, for instance with the Euler characteristic of $\Sigma$ and the dimension of $\Sigma$ ) and on the spectra of operators [17]. This opens the possibility to describe topology change within canonical quantum gravity.

\section{The Gauss Constraint}

In this section we implement the Gauss constraint into the theory. On $(M, \Omega)$ it is given by the function $\left(\Lambda^{i} \in \mathcal{S}\right)$

$$
G(\Lambda):=\int_{\Sigma} d^{D} x \Lambda^{i}(x)\left[\partial_{a} E_{i}^{a}(x)+f_{i j}^{k} A_{a}^{j}(x) E_{k}^{a}(x)\right]
$$

which generates infinitesimal gauge transformations

$$
\begin{aligned}
F(A) & \mapsto F(A)+\{F(A), G(\Lambda)\}=F(A+D \Lambda) \\
E(f) & \mapsto E(f)+\{E(f), G(\Lambda)\}=E(f+[\Lambda, f])
\end{aligned}
$$


where $\Lambda=\Lambda^{i} \tau_{i} / 2, A=A^{i} \tau_{i} / 2, E=E_{i} \tau_{i} / 2, f=f^{i} \tau_{i} / 2, F=F_{i} \tau_{i} / 2$. The maps (4.2) are the infinitesimal versions of the finite gauge transformations

$$
\begin{aligned}
F(A) & \mapsto F\left(\mathrm{Ad}_{g} \cdot A-d g g^{-1}\right) \\
E(f) & \mapsto\left[\operatorname{Ad}_{g} \cdot E\right](f)
\end{aligned}
$$

where $\operatorname{Ad}_{g} \cdot v:=g v g^{-1}$ is the adjoint representation of $G$ on $\operatorname{Lie}(G)$. Indeed, for infinitesimal $\Lambda$, (4.3) reproduces (4.2) to linear order provided we identify $g(x)=\exp \left(-\Lambda^{i}(x) \tau_{i} / 2\right)$.

Our task is to write (4.1) in terms of $\left(M_{\gamma}, \Omega_{\gamma}\right)$. That is, we must find a function $G_{\gamma}(\Lambda)$ on $M_{\gamma}$ such that it converges pointwise on $M$ to $G(\Lambda)$ and such that the limit of its Poisson brackets, that is, its Hamiltonian vector field on $M_{\gamma}$ converges to the Hamiltonian vector field of $G(\Lambda)$ on $M$.

To do this we will proceed as follows :

1) Find the gauge transformations of the coordinates $h_{e}, P^{e}$ of $M_{\gamma}$ induced by (4.2).

2) Find a generator $G_{\gamma}(\Lambda)$ on $M_{\gamma}$ of these infinitesimal transformations.

3) Study the generator and its Hamiltonian vector field on $M_{\gamma}$ and consider the limit $\gamma \rightarrow \Sigma$.

Notice that this procedure works only because gauge transformations have the special feature to preserve $M_{\gamma}$ as we will see. This is not the case for more general gauge groups such as diffeomorphisms which map between different $M_{\gamma}$ 's and which are relevant for quantum general relativity 28.

It is immediate from the definition of a principal fibre bundle with connection over $\Sigma$ and an associated (under the adjoint representation of $G$ ) vector bundle that under finite gauge transformations $x \in \Sigma \mapsto g(x) \in G$

$$
\begin{aligned}
h_{e}(A) & \mapsto g(e(0)) h_{e}(A) g(e(1))^{-1} \\
P^{e}(A, E) & \mapsto g(e(0)) P^{e}(A, E) g(e(0))^{-1}=: \operatorname{Ad}_{g(e(0))} \cdot P^{e}(A, E)
\end{aligned}
$$

where $P^{e}=P_{i}^{e} \tau_{i} / 2$. This follows from the manifestly gauge covariant definition of the basic coordinates of $M_{\gamma}$ given in (3.15). The infinitesimal version of (4.4) is given by (with $g(x)=$ $\left.\exp \left(-\Lambda^{i}(x) \tau_{i} / 2\right)=\exp (-\Lambda(x))\right)$

$$
\begin{aligned}
h_{e} & \mapsto h_{e}-\Lambda(e(0)) h_{e}-h_{e} \Lambda(e(1)) \\
P^{e} & \mapsto P^{e}+\left[P^{e}, \Lambda(e(0))\right]
\end{aligned}
$$

which should equal $\left\{h_{e}, G_{\gamma}(\Lambda\}_{\gamma},\left\{P^{e}, G_{\gamma}(\Lambda)\right\}_{\gamma}\right.$ respectively.

It is immediately clear from (3.26) that the second line of (4.5) can be obtained by choosing

$$
G_{\gamma}(\Lambda)=\sum_{v \in V(\gamma)} \Lambda^{i}(v) \sum_{e \in E(\gamma), e(0)=v} P_{i}^{e}+\text { more }
$$

where "more" should commute with all the $P_{i}^{e}$. Ansatz (4.6) already correctly reproduces also the $\Lambda(e(0))$ term of the first line of (4.5), the $\Lambda(e(1))$ term looks similar just that it corresponds to an insertion of $\tau_{i}$ from the right instead of from the left. Since the holonomies Poisson commute among themselves we are led to the following improved ansatz

$$
G_{\gamma}(\Lambda)=\sum_{v \in V(\gamma)} \Lambda^{i}(v)\left[\sum_{e \in E(\gamma), e(0)=v} P_{i}^{e}+\sum_{e \in E(\gamma), e(1)=v} M_{i j}\left(h_{e}\right) P_{j}^{e}\right]
$$

where the matrix $M_{i j}\left(h_{e}\right)$ should satisfy $-M_{i j}\left(h_{e}\right) \tau_{j} h_{e}=h_{e} \tau_{i}$ and $\left\{P_{i}^{e}, M_{j k}\left(h_{e}\right) P_{k}^{e}\right\}_{\gamma}=0$. The first requirement leads to the unique solution

$$
M_{i j}(h)=\frac{1}{N} \operatorname{tr}\left(h \tau_{i} h^{-1} \tau_{j}\right)
$$


while the second asks us to check the vanishing of (use $\left\{., h^{-1}\right\}_{G}=-h^{-1}\{., h\}_{G} h^{-1}$ )

$$
\begin{aligned}
\left\{P_{i}, M_{j k}(h) P_{k}\right\}_{G} & =-M_{j k}(h) f_{i k}{ }^{l} P_{l}+\left\{P_{i}, M_{j l}(h)\right\}_{G} P_{l} \\
& =\left[-M_{j k}(h) f_{i k}{ }^{l}+\frac{1}{N} \operatorname{tr}\left(\frac{\tau_{i}}{2} h \tau_{j} h^{-1} \tau_{l}-h \tau_{j} h^{-1} \frac{\tau_{i}}{2} \tau_{l}\right)\right] P_{l} \\
& =\left[-f_{i k}{ }^{l}+f_{l i}{ }^{k}\right] M_{j k}(h) P_{l}
\end{aligned}
$$

which indeed vanishes for $G$ semisimple as we assume.

We notice that

$$
P_{i}^{e \prime}:=-M_{i j}\left(h_{e}\right) P_{j}^{e}=P_{i}^{e^{-1}}
$$

which explains intuitively why it is possible that $\left\{P_{i}^{e}, P_{j}^{e^{\prime \prime}}\right\}_{\gamma}$ vanishes for any $e^{\prime}$ : while $P^{e}$ depends only on the beginning half segment of the edge $e, P^{e \prime}$ depends only on the ending half segment of the edge $e$ and given the symplectic structure (3.13) a non-vanishing bracket is therefore impossible (modulo the regularization procedure of section 3.2.2). Of course, in retrospect the result should have been guessed on general grounds as what we were trying to construct are the generators $P, P^{\prime}$ respectively of left and right translations respectively on $G$ which, of course, commute.

In order to check the continuum limit of the function (4.7) we employ the sequence of graphs $\gamma_{n}$ of section 3.2.3. Using the notation of that section, in particular (3.49), we define for $v \in \gamma_{R}, R \in \mathcal{R}$ the quantity

$$
E^{I}(v)=h_{e_{I}(v)}(0,1 / 2)^{-1}\left[\int_{S^{I}(v)} h_{\rho_{e_{I}(v)}(x)} * E(x) h_{\rho_{e_{I}(v)}^{-1}(x)}\right] h_{e_{I}(v)}(0,1 / 2)
$$

which to order $\epsilon_{n}^{D-1}$ equals $\epsilon_{n}^{D-1} n_{a}^{I}(v) E_{i}^{a}(v) \tau_{i}$ as $n \rightarrow \infty$. Then for fixed $n$

$$
\begin{aligned}
& G_{\gamma_{n}}(\Lambda)=\sum_{R \in \mathcal{R}} \sum_{v \in V\left(\gamma_{R}\right)} \Lambda^{i}(v)\left[\sum_{e \in E\left(\gamma_{R}\right), v=e(0)} P_{i}^{e}+\sum_{e \in E\left(\gamma_{R}\right), v=e(1)} M_{i j}\left(h_{e}\right) P_{j}^{e}\right] \\
= & \sum_{R \in \mathcal{R}} \sum_{v \in V\left(\gamma_{R}\right)} \sum_{I=1}^{D}\left[P_{i}^{I}(v) \Lambda^{i}(v)+M_{i j}\left(h_{I}(v)\right) P_{j}^{I}(v) \Lambda^{i}\left(X_{R}\left(X_{R}^{-1}(v)+\epsilon b_{I}\right)\right)\right] \\
= & \sum_{R \in \mathcal{R}} \sum_{v \in V\left(\gamma_{R}\right)} \Lambda^{i}(v) \sum_{I=1}^{D}\left[P_{i}^{I}(v)+M_{i j}\left(h_{I}\left(X_{R}\left(X_{R}^{-1}(v)-\epsilon b_{I}\right)\right)\right) P_{j}^{I}\left(X_{R}\left(X_{R}^{-1}(v)-\epsilon b_{I}\right)\right)\right] \\
= & -\frac{1}{N} \sum_{R \in \mathcal{R}} \sum_{v \in V\left(\gamma_{R}\right)} \Lambda^{i}(v) \sum_{I=1}^{D} \operatorname{tr}\left(\tau_{i}\left[h_{I}(v) E^{I}(v) h_{I}(v)^{-1}-E^{I}\left(X_{R}\left(X_{R}^{-1}(v)-\epsilon b_{I}\right)\right)\right]\right. \\
= & -\frac{1}{N} \sum_{R \in \mathcal{R}} \sum_{v \in V\left(\gamma_{R}\right)} \Lambda^{i}(v) \sum_{I=1}^{D} \operatorname{tr}\left(\tau _ { i } \left[\left\{h_{I}(v) E^{I}(v) h_{I}(v)^{-1}-E^{I}(v)\right\}\right.\right. \\
& \left.\left.+\left\{E^{I}(v)-E^{I}\left(X_{R}\left(X_{R}^{-1}(v)-\epsilon b_{I}\right)\right)\right\}\right]\right)
\end{aligned}
$$

Consider the two curly brackets in the last line of (4.11). The first one is given to leading order $\epsilon^{D}$ by $Y_{I}^{b}(v) n_{a}^{I}(v)\left[A_{b}(v), E^{a}(v)\right]=\operatorname{det}\left(Y_{R}\right)(v)\left[A_{a}(v), E^{a}(v)\right]$. The second one is given to leading order $\epsilon_{n}^{D}$ by

$$
\left[\frac{\partial\left(n_{a}^{I}\left(X_{R}(t)\right) E^{a}\left(X_{R}(t)\right)\right)}{\partial t^{I}}\right]_{X_{R}(t)=v}=n_{a}^{I}(v) Y_{I}^{b}(v) \partial_{b} E^{a}(v)=\operatorname{det}\left(Y_{R}\right)(v) \partial_{a} E^{a}(v)
$$

since $\sum_{I} \partial_{I} n_{a}^{I}\left(X_{R}(t)\right)=0$. Now the sum of the differences $h_{I}(v) E^{I}(v) h_{I}(v)^{-1}-E^{I}(v)-\epsilon_{n}^{D} \operatorname{det}\left(Y_{R}\right)(v)\left[A_{a}(v), E^{a}(v)\right]$ and 
$E^{I}(v)-E^{I}\left(X_{R}\left(X_{R}^{-1}(v)-\epsilon b_{I}\right)\right)-\epsilon_{n}^{D} \operatorname{det}\left(Y_{R}\right)(v) \partial_{a} E^{a}(v)$ can be written as $\epsilon_{n}^{D+1} \operatorname{det}\left(Y_{R}\right)(v) K(v)$ where $K$ is an integrable function. Thus, (4.11) becomes

$$
\begin{aligned}
G_{\gamma_{n}}(\Lambda)= & \sum_{R \in \mathcal{R}} \sum_{v \in V\left(\gamma_{R}\right)} \epsilon_{n}^{D} \operatorname{det}\left(Y_{R}\right)(v) \Lambda^{i}(v)\left(\partial_{a} E_{i}^{a}+f_{i j}{ }^{k} A_{a}^{j} E_{k}^{a}\right)(v) \\
& -\frac{\epsilon_{n}}{N} \sum_{R \in \mathcal{R}} \sum_{v \in V\left(\gamma_{R}\right)} \epsilon_{n}^{D} \operatorname{det}\left(Y_{R}\right)(v) \Lambda^{i}(v) \sum_{I=1}^{D} \operatorname{tr}\left(\tau_{i} K(v)\right)
\end{aligned}
$$

and both sums are Riemann sum approximations of integrals. Recall that $\operatorname{det}\left(Y_{R}\right)(v)>0$ for $v \in R$ and that $\epsilon_{n}^{D} \operatorname{det}(Y)(v)$ approximates the Lebesgue measure of the image under $X_{R}$ of a cube of volume $\epsilon$ in $V_{R}$. It follows that

$$
\lim _{n \rightarrow \infty} G_{\gamma_{n}}(\Lambda)=G(\Lambda)-\lim _{n \rightarrow \infty} \frac{\epsilon_{n}}{N} \int_{\Sigma} d^{D} x \Lambda^{i}(x) \operatorname{tr}\left(\tau_{i} K(x)\right)=G(\Lambda)
$$

as desired.

To check that also the Hamiltonian vector field of $G_{\gamma}(\Lambda)$ converges to the one of $G(\Lambda)$ we consider the brackets defined by

$$
\begin{aligned}
& \{F(A), G(\Lambda)\}^{\prime}:=\lim _{n \rightarrow \infty}\left\{F \cdot A^{(n)}, G_{\gamma_{n}}(\Lambda)\right\}_{\gamma_{n}} \\
& \{E(f), G(\Lambda)\}^{\prime}:=\lim _{n \rightarrow \infty}\left\{E^{(n)} \cdot f, G_{\gamma_{n}}(\Lambda)\right\}_{\gamma_{n}}
\end{aligned}
$$

where $A^{(n)}, E^{(n)}$ are defined in (3.51), (3.52). Using the definitions and reasonings repeatedly outlined already in this paper we see that indeed $\{F(A), G(\Lambda)\}^{\prime}=\{F(A), G(\Lambda)\}$ and $\{E(f), G(\Lambda)\}^{\prime}=\{E(f), G(\Lambda)\}$. So, the Hamiltonian vector fields also coincide in the limit $\gamma \rightarrow \Sigma$ and display (4.7) as a satisfactory discretization of $G(\Lambda)$.

\section{Quantization}

In this section we quantize all the phase spaces $\left(M_{\gamma}, \Omega_{\gamma}\right)$. Notice that in the literature so far one quantized either $(M, \Omega)$ [10] or one quantized only one particular family of $\left(M_{\gamma}, \Omega_{\gamma}\right)$ 's that were defined through lattices in $\Sigma$ 's of the topology of $\mathbb{R}^{3}$ [55]. In the former case one took a classical function and tried to turn it into an operator after going through some regularization and renormalization steps. In the latter case one started directly with some operators and required that they have a certain continuum limit behaviour with respect to the lattice spacing, however, one did not establish a precise relation between these discrete operators and certin smeared objects of the continuum theory as we did in section 3. However, without such an analysis it is quite unclear what the operators so obtained do actually measure. In particular, one has to postulate the $\epsilon$ expansion of the $P^{e}, h_{E}$ rather than being able to derive it from first principles.

By definition, quantization means to find an irreducible representation of an algebra of operators $\hat{h}_{e}, \hat{P}_{e}$ such that the symplectic and the reality structure of the classical theory is correctly implemented. More concretely, since $M_{\gamma}$ is isomorphic with the direct product of co-tangent bundles $T^{*} G$, one copy for each edge of $\gamma$, it is suggested to choose the natural real polarization of the phase space in which wave functions depend only on holonomies. Thus we choose a Hilbert space $\mathcal{H}_{\gamma}$ of square integrable functions of the $h_{e}, e \in E(\gamma)$ with respect to a measure $\mu_{\gamma}$, that is, $\mathcal{H}_{\gamma}=L_{2}\left(\mathcal{C}_{\gamma}, d \mu_{\gamma}\right)$ where $\mathcal{C}_{\gamma}=G^{|E(\gamma)|}$ is the complete quantum (and also classical in the absence of boundary conditions) configuration space and must represent the operators $\hat{h}_{e}^{A B}, \hat{P}_{i}^{e}$ on $\mathcal{H}_{\gamma}$ in such a way that the following commutation relations hold :

$$
\left[\hat{h}_{e}^{A B}, \hat{h}_{e^{\prime}}^{C D}\right]=0
$$




$$
\begin{aligned}
{\left[\hat{P}_{j}^{e}, \hat{h}_{e^{\prime}}^{A B}\right] } & =i \hbar \delta_{e^{\prime}}^{e}\left(\frac{\tau_{j}}{2} \hat{h}_{e}\right)^{A B} \\
{\left[\hat{P}_{j}^{e}, \hat{P}_{k}^{e^{\prime}}\right] } & =i \hbar \delta^{e e^{\prime}}\left(-f_{j k}{ }^{l}\right) \hat{P}_{l}^{e}
\end{aligned}
$$

More precisely, we must find a common dense domain $\mathcal{D}_{\gamma}$ of all the basic operators which they leave invariant so that it makes sense to compute commutators. Notice again that we allow the graph $\gamma$ to be infinite.

Furthermore, the reality structure of the classical theory is given by (let us choose $G$ to be a (subgroup of a) unitary group for definiteness)

$$
\overline{h_{e}^{A B}}=\left(h_{e}^{-1}\right)^{B A} \text { and } \overline{P_{i}^{e}}=P_{i}^{e}
$$

To see the latter, notice that from (3.15) $P_{i}^{e}$ is given by an integral of quantities of the form $\operatorname{tr}\left(g \tau_{i} g^{-1} \tau_{j}\right) v^{j}$ where $v^{j}$ is real and $g \in G$. From $\bar{g}^{T} g=1$ it follows with $g=\exp \left(\theta^{j} \tau_{j} / 2\right), \theta^{j}$ real that $\bar{\tau}_{j}^{T}=-\tau_{j}$. Therefore the orthogonal matrix, using $\operatorname{tr}\left(M^{T}\right)=\operatorname{tr}(M)$,

$$
O_{i j}(g):=-\frac{1}{N} \operatorname{tr}\left(g \tau_{i} g^{-1} \tau_{j}\right)
$$

is real.

In conclusion we must impose the following adjointness relations on $\mu_{\gamma}$

$$
\left(\hat{h}_{e}^{A B}\right)^{\dagger}=\left(\widehat{h}_{e}^{-1}\right)^{B A} \text { and }\left(\hat{P}_{i}^{e}\right)^{\dagger}=\hat{P}_{i}^{e}
$$

where the first identity has to be understood in the sense that one should write the function $h_{e}^{-1}$ in terms of $h_{e}$ and then replace it by $\hat{h}_{e}$. No operator ordering problems arise since the $h_{e}^{A B}$ are mutually commuting. As advertized, we will choose the $\hat{h}_{e}^{A B}$ as multiplication operators with values in $G$. As $G$ is compact, these operators are bounded and thus they are defined, together with $\left(\hat{h}_{e}^{-1}\right)^{A B}$, everywhere on $\mathcal{H}_{\gamma}$ so that there are no domain questions at all in the definition of $\left(\hat{h}_{e}^{A B}\right)^{\dagger}$. The second identity in (5.4) says that $\hat{P}_{j}^{e}$ is a self-adjoint operator and in order to settle the domain question we will determinine an explicit core $\mathcal{D}_{\gamma}$ of essential self-adjointness for all the $\hat{P}_{i}^{e}$.

Let us choose as $\mathcal{D}_{\gamma}:=C^{\infty}\left(\mathcal{C}_{\gamma}\right)$ where we consider $\mathcal{C}_{\gamma}$ as a Banach manifold modelled on $\mathbb{R}^{\operatorname{dim}(G)|E(\gamma)|}$ similar as for $M_{\gamma}$. Then we choose the following action of the basic operators on $f_{\gamma} \in \mathcal{D}_{\gamma}$

$$
\begin{aligned}
\left(\hat{h}_{e}^{A B} f_{\gamma}\right)\left(\left\{h_{e^{\prime}}\right\}\right) & :=h_{e}^{A B} f_{\gamma}\left(\left\{h_{e^{\prime}}\right\}\right) \\
\left(\hat{P}_{j}^{e} f_{\gamma}\right)\left(\left\{h_{e^{\prime}}\right\}\right) & :=\frac{i \hbar}{2}\left(X_{j}^{e} f_{\gamma}\right)\left(\left\{h_{e^{\prime}}\right\}\right)
\end{aligned}
$$

where $X_{j}^{e}=X\left(h_{e}\right)_{j}, X(g)_{i}:=\operatorname{tr}\left(\left(\tau_{j} g\right)^{T} \partial / \partial g\right)$ denotes the right invariant vector field on $G$ (the generator of left translations). First of all, the operations (5.5) leave $\mathcal{D}_{\gamma}$ obviously invariant. Next we have the Lie algebra of vector fields on $G$ given by $\left[X_{j}, X_{k}\right]=-2 f_{j k}{ }^{l} X_{l}$ and it is easy to see that with this choice the commutation relations (5.1) are identically satisfied.

The direct product structure of $\mathcal{C}_{\gamma}$ shows that we may choose

$$
d \mu_{\gamma}\left(\left\{h_{e}\right\}_{e \in E(\gamma)}\right)=\otimes_{e \in E(\gamma)} d \mu_{e}\left(h_{e}\right)
$$

and in order that the adjointness relations (5.4) be satisfied it will be sufficient to choose $\mu_{e}=\mu_{G}$ for all $e \in E(\gamma)$. Let $\mathcal{H}_{G}=L_{2}\left(G, d \mu_{G}\right)$, then we must establish the symmetry property

$$
<f, i X_{j} f^{\prime}>_{G}=i \int_{G} d \mu_{G}(h) \overline{f(h)}\left(X_{j} f^{\prime}\right)(h)=<i X_{j} f, f^{\prime}>_{G}
$$


for all $f, f^{\prime} \in C^{\infty}(G)$. Notice that $\mathcal{D}\left(X^{\dagger}\right)$, the set of elements $f \in \mathcal{H}_{G}$ for which the map $\psi \mapsto<f, i X_{j} \psi>$ defines a continuous linear functional on $\mathcal{D}(X)=C^{\infty}(G)$, certainly contains $\mathcal{D}(X)$ so that (5.6) implies symmetry.

Now $X_{j}$ generates left translations and $\partial G=\emptyset$, thus, if we choose the measure $\mu_{G}$ to be left invariant we are done provided we can establish that the expression for $X_{j}$ is real valued. For compact groups the only solution is, up to a normalization, $\mu_{G}=\mu_{H}$, the Haar measure on $G$ which is simultaneously left and right invariant and normalized, $<1,1>_{G}=1$. To see that the expression for $X_{j}$ is real we perform the following calculation :

$$
\begin{aligned}
<f, X_{j} f^{\prime}>_{G} & =\int_{G} d \mu_{H}(h) \overline{f(h)} \frac{d}{d t} f_{t=0}^{\prime}\left(e^{t \tau_{j} / 2} h\right) \\
& =\frac{d}{d t} \int_{t=0} d \mu_{H}(h) \overline{f(h)} f^{\prime}\left(e^{t \tau_{j} / 2} h\right) \\
& =\frac{d}{d t} \int_{t=0} d \mu_{H}\left(e^{-t \tau_{j} / 2} h\right) \overline{f\left(e^{-t \tau_{j} / 2} h\right)} f^{\prime}(h) \\
& =-\frac{d}{d t} \int_{t=0} d \mu_{G}(h) \overline{f\left(e^{t \tau_{j} / 2} h\right)} f^{\prime}(h) \\
& =-<X_{j} f, f^{\prime}>_{G}
\end{aligned}
$$

as claimed.

Finally, to see that $i X_{j}$ is essentially self-adjoint with core $\mathcal{D}(X)=C^{\infty}(G)$ we show that $\left[X_{j} \pm\right.$ $\left.\operatorname{id}_{\mathcal{H}_{G}}\right] \mathcal{D}_{G}$ is dense in $\mathcal{H}_{G}$ (basic criterion of essential self-adjointness). The proof is simplified through an appeal to the Peter\&Weyl theorem [56]: the Hilbert space $\mathcal{H}_{G}$ is the completion of a countable orthogonal sum of finite dimensional Hilbert spaces $\mathcal{H}_{\pi}$ where $\pi$ runs through the set of equivalence classes of irreducible representations of $G$. A complete orthonormal basis of $\mathcal{H}_{\pi}$ is given by the functions $\sqrt{d_{\pi}} \pi_{m n}(h)$ where $d_{\pi}$ is the dimension of the representation and $\pi_{m m^{\prime}}$ denotes the matrix elements of an abrbitary but fixed representant of that equivalence class. These functions obviously belong to $\mathcal{D}(X)$ and finite linear combinations of such functions are still in $\mathcal{D}(X)$. Thus, the finite linear combinations of such functions belong to the domain, $\oplus_{\pi} \mathcal{H}_{\pi} \subset \mathcal{D}(X)$.

Next, it is easy to see that $X_{j}$ preserves $\mathcal{H}_{\pi}$. Denote by $X_{j}^{\pi}$ the restriction of $X_{j}$ to $\mathcal{H}_{\pi}$ then $i X_{j}^{\pi}$ is a symmetric operator on the finite dimensional Hilbert space $\mathcal{H}_{\pi}$ and therefore self-adjoint on $\mathcal{H}_{\pi}$ with domain given by all of $\mathcal{H}_{\pi}$. By the basic criterion for self-adjointness, $\left[X_{j}^{\pi} \pm 1\right] \mathcal{H}_{\pi}=\mathcal{H}_{\pi}$. The proof is then complete with the observation that

$$
\begin{aligned}
& {\left[X_{j} \pm 1\right] \oplus_{\pi} \mathcal{H}_{\pi}=\oplus_{\pi}\left(\left[X_{j}^{\pi} \pm 1\right] \mathcal{H}_{\pi}\right)=\oplus_{\pi} \mathcal{H}_{\pi} \subset\left[X_{j} \pm 1\right] \mathcal{D}(X) } \\
\Rightarrow \quad & \mathcal{H}_{G}=\overline{\oplus_{\pi} \mathcal{H}_{\pi}} \subset \overline{\left[X_{j} \pm 1\right] \mathcal{D}(X)} \subset \mathcal{H}_{G}
\end{aligned}
$$

Remark :

To see that $X_{j}^{\pi}$ does not have real eigenvectors in a more elementary way, recall that $\left(X_{j}^{\pi}\right)^{2}=$ $-\lambda_{\pi}<0$ is the Laplacian on $G$.

In conclusion the (possibly infinite) tensor product of Hilbert spaces

$$
\mathcal{H}_{\gamma}:=\otimes_{e \in E(\gamma)} \mathcal{H}_{e}=L_{2}\left(\mathcal{C}_{\gamma}, d \mu_{0 \gamma}=\otimes_{e \in E(\gamma)} d \mu_{e}\right)
$$

where each of the $\mathcal{H}_{e}$ is isomorphic with $L_{2}\left(G, d \mu_{H}\right)$ is a faithful representation of the canonical commutation relations (5.1) and of the adjointness relations (5.4). Moreover, given the action (5.5), it is easy to see that the product Haar measure $\mu_{0 \gamma}$ is the unique solution, that is, any other measure $\mu_{\gamma}$ which is regular with respect to it must be a constant multiple of it. Notice that infinite products of probability measures are well-defined and $\sigma$-additive probability measures by the Kolmogorov theorem [57]. Much more will be said about infinite tensor products 
of Hilbert spaces in the first reference of 44 .

We now must quantize various functions on $M_{\gamma}$. It is at this point where our detailed analysis becomes crucial : while in the continuum theory important functions such as the Gauss constraint (4.1) are written as integrals over polynomials of the field variables $A(x), E(x)$ at the same point, that is, not as polynomials of the smeared functions $F(A), E(f)$, the functions on $M_{\gamma}$ are polynomials of the basic observables $h_{e}, P^{e}$ which are already smeared. Thus, while the quantization of, say, $G(\Lambda)$ on $(M, \Omega)$ can possibly produce UV divergent objects, the quantization of $G_{\gamma}(\Lambda)$ on $\left(M_{\gamma}, \Omega_{\gamma}\right)$ cannot suffer from such problems. (Of course, in both cases factor ordering problems might appear but in the latter case this is only an ambiguity and not the source of a divergence). One might think that problems occur when taking the limit $\gamma \rightarrow \Sigma$, but as we will show, this does not happen.

On the other hand, in both cases we can still have IR divergencies. However, again, from our point of view this is not a problem at all! Namely, our operator is densely defined, that is, it is an unbounded operator defined on a dense domain. This dense subset of the Hilbert space, however, does not contain states with infinite volume. Nevertheless it is possible to deal with this situation appropriately 44. In contrast, the perturbative quantization of general relativity is based on a cyclic vector with infinite volume and therefore IR divergencies necessarily occur.

Let us then quantize the Gauss constraint $G_{\gamma}(\Lambda)$. We choose to order the momentum variables to the right of the configuration variables and obtain

$$
\hat{G}_{\gamma}(\Lambda)=\sum_{e \in E(\gamma)}\left[\Lambda^{i}(e(0)) \delta_{i j}-\Lambda^{i}(e(1)) O_{i j}\left(\hat{h}_{e}\right)\right] \hat{P}_{j}^{e}
$$

Let us check that there are no quantum anomalies. First of all we compute the classical constraint algebra. We have

$$
\begin{aligned}
\left\{O_{i j}(h) P_{j}, O_{k l}(h) P_{l}\right\}_{G} & =\left\{O_{i j}(h), O_{k l}(h) P_{l}\right\}_{G} P_{j}=O_{k l}(h)\left\{O_{i j}(h), P_{l}\right\}_{G} P_{j} \\
& =\frac{1}{N} O_{k l}(h) \operatorname{tr}\left(\frac{\tau_{l}}{2} h \tau_{i} h^{-1} \tau_{j}-h \tau_{i} h^{-1} \frac{\tau_{l}}{2} \tau_{j}\right) P_{j}=-f_{j l}{ }^{m} O_{k l}(h) O_{i m}(h) P_{j}
\end{aligned}
$$

Now since

$$
h \tau_{i} h^{-1}=\operatorname{Ad}_{h} \cdot \tau_{i}=O_{i j}(h) \tau_{j}
$$

we have the identity

$$
\begin{aligned}
{\left[\operatorname{Ad}_{h} \cdot \tau_{i}, \operatorname{Ad}_{h} \cdot \tau_{j}\right] } & =O_{i k}(h) O_{j l}(h)\left[\tau_{k}, \tau_{l}\right]=\operatorname{Ad}_{h} \cdot\left[\tau_{i}, \tau_{j}\right] \\
\Rightarrow \quad O_{i k}(h) O_{j l}(h) f_{k l}{ }^{m} & =f_{i j}{ }^{k} O_{k m}(h)
\end{aligned}
$$

which, when inserted into (5.11), gives

$$
\left\{O_{i j}(h) P_{j}, O_{k l}(h) P_{l}\right\}_{G}=-f_{l m}{ }^{j} O_{k l}(h) O_{i m}(h) P_{j}=f_{i k}{ }^{m} O_{m j}(h) P_{j}
$$

or, recalling (4.10),

$$
\left\{P_{i}^{e \prime}, P_{j}^{e^{\prime \prime}}\right\}_{\gamma}=\delta^{e e^{\prime}} f_{i j}{ }^{k} P_{k}^{e \prime}
$$

which is the algebra of left invariant vector fields on $G$ (notice the relative minus sign as compared to 3.26$)$.

Thus, since the $P_{i}^{e}, P_{j}^{e^{\prime \prime}}$ Poisson commute by definition of the matrix $O_{i j}=-M_{i j}$ we immediately get with

$$
G_{\gamma}(\Lambda)=\sum_{v \in V(\gamma)} \Lambda^{i}(v)\left[\sum_{e(0)=v} P_{i}^{e}-\sum_{e(1)=v} P_{i}^{e \prime}\right]
$$

that

$$
\left\{G_{\gamma}(\Lambda), G_{\gamma}\left(\Lambda^{\prime}\right)\right\}_{\gamma}=\sum_{v \in V(\gamma)} \Lambda^{i}(v) \Lambda^{j \prime}(v) f_{i j}{ }^{k}\left[-\sum_{e(0)=v} P_{k}^{e}+\sum_{e(1)=v} P_{k}^{e \prime}\right]=-G_{\gamma}\left(\left[\Lambda, \Lambda^{\prime}\right]\right)
$$


as desired because we infer from (4.2) that the continuum Poisson algebra is given by (use the Jacobi identity)

$$
\begin{aligned}
& \left\{\{E(f), G(\Lambda)\}, G\left(\Lambda^{\prime}\right)\right\}-\left\{\left\{E(f), G\left(\Lambda^{\prime}\right)\right\}, G(\Lambda)\right\}=\left\{E(f),\left\{G(\Lambda), G\left(\Lambda^{\prime}\right)\right\}\right\} \\
= & E\left(\left[\Lambda^{\prime},[\Lambda, f]\right]-\left[\Lambda,\left[\Lambda^{\prime}, f\right]\right]\right)=E\left(\left[f,\left[\Lambda^{\prime}, \Lambda\right]\right]\right)=-\left\{E(f), G\left(\left[\Lambda, \Lambda^{\prime}\right]\right)\right\}
\end{aligned}
$$

Thus, the algebra (5.17) converges to (5.18) by (4.13).

Now, it follows trivially from (5.1), (5.10) that

$$
\left[\hat{G}_{\gamma}(\Lambda), \hat{G}_{\gamma}\left(\Lambda^{\prime}\right)\right]=i \hbar\left(-\hat{G}_{\gamma}\left(\left[\Lambda, \Lambda^{\prime}\right]\right)\right)
$$

as required.

Let us summarize :

We started with a continuum phase space $(M, \Omega)$ and derived from it a discrete phase space $\left(M_{\gamma}, \Omega_{\gamma}\right)$ for every graph $\gamma$. We also showed that $(M, \Omega)$ is the pointwise limit of $\left(M_{\gamma}, \Omega_{\gamma}\right)$ as $\gamma \rightarrow \Sigma$. Next, we took the Gauss constraint $G(\Lambda)$ which is a function on $M$ and derived from it a function $G_{\gamma}(\Lambda)$ on $M_{\gamma}$ which again converges pointwise to $G(\Lambda)$. Moreover, the Poisson algebra of the $G_{\gamma}(\Lambda)$ with respect to $\Omega_{\gamma}$ closes for every fixed $\gamma$ and converges pointwise to the Poisson algebra of the $G(\Lambda)$. Finally, we quantized the $G_{\gamma}(\Lambda)$ and obtained an anomaly free algebra of quantum constraints $\hat{G}_{\gamma}(\Lambda)$. Then two questions remain :

1.) Does this structure provide us with a quantization of $G(\Lambda)$ as well ? That is, can we find an operator $\hat{G}(\Lambda)$ densely defined on all of $\mathcal{H}$ and not only on $\mathcal{H}_{\gamma}$ such that

$$
\hat{G}(\Lambda) f_{\gamma}=\hat{G}_{\gamma}(\Lambda) f_{\gamma}
$$

for every function $f_{\gamma}$ cylindrical over a graph $\gamma$ ?

2.) If $\hat{G}(\Lambda)$ exists, does its classical limit coincide with the classical function $G(\Lambda)$ ?

[1.]

It is easy to see that the first question can be answered affirmatively :

Namely, in order that (5.20) holds it is sufficient to show that the family of operators $\hat{G}_{\gamma}(\Lambda)$ is consistently defined. But this is trivially the case because we defined a function to be cylindrical over $\gamma$ if and only if it is a finite linear combination of spin-network functions which by definition depend non-trivially on the holnomy along each of its edges (that is, each edge is labelled with a non-trivial irreducible representation of $G$ ). Thus, if we superpose $f_{\gamma}, f_{\gamma^{\prime}}^{\prime}$ with $\gamma \neq \gamma^{\prime}$ then $G(\Lambda)\left[f_{\gamma}+f_{\gamma^{\prime}}^{\prime}\right]:=G_{\gamma}(\Lambda) f_{\gamma}+G_{\gamma^{\prime}}(\Lambda) f_{\gamma^{\prime}}^{\prime}$. It is also easy to see that this definition leads to the constraint algebra

$$
\left[\hat{G}(\Lambda), \hat{G}\left(\Lambda^{\prime}\right)\right]=i \hbar\left(-\hat{G}\left([\Lambda), \Lambda^{\prime}\right]\right)
$$

by (5.19) since $\hat{G}_{\gamma}(\Lambda)$ preserves $\mathcal{H}_{\gamma}$. Thus, $\hat{G}(\Lambda)$ exists and defines a consistent quantum constraint algebra.

[2.]

To address the second question we first of all notice that we have shown that

$$
G(\Lambda)=\lim _{\gamma \rightarrow \Sigma}\left[\lim _{\hbar \rightarrow 0} \hat{G}_{\gamma}(\Lambda)\right]
$$

where the inner bracket has been demonstrated actually only by the usual "quantization rule". A rigorous proof will be given elesewhere [42, 43], see also below for a sketch. The outer limit is to be understood pointwise on $M$.

What we would like to establish now is the existence of the opposite limiting procedure, that is

$$
G(\Lambda)=\lim _{\hbar \rightarrow 0}\left[\lim _{\gamma \rightarrow \Sigma} \hat{G}_{\gamma}(\Lambda)\right]
$$


We will understand the inner bracket to be the operator $\hat{G}(\Lambda)$ defined in (5.19) through the self-consistent family of projections $\hat{G}_{\gamma}(\Lambda)$.

We can then rigorously define the limits $(5.22)$ and $(5.23)$ as follows :

Let $\psi_{\gamma, m}^{\hbar}$ be a coherent state, explicitly dependent on Planck's constant, peaked at the point $m \in M$ (a smooth field configuration) in the following sense : For each graph $\gamma$ and its associated dual decomposition $P_{\gamma}$ consider the values of the holonomies and momenta $h_{e}(m), P^{e}(m)$ respectively. Then the operators $\hat{h}_{e}, \hat{P}^{e}$ have expectation values $h_{e}(m), P^{e}(m)$ in the state $\psi_{\gamma}^{m}$ respectively and satisfy a minimal uncertainty condition.

Let now $\gamma_{n}$ be the family of graphs defined in section 3.2.3. We then consider the expectation values

$$
G_{n}^{\hbar}(\Lambda, m):=<\psi_{\gamma_{n}, m}^{\hbar}, \hat{G}_{\gamma_{n}}(\Lambda) \psi_{\gamma_{n}, m}^{\hbar}>_{\gamma_{n}}
$$

Notice that by definition of the Hilbert space $\mathcal{H}$ and the operator $\hat{G}(\Lambda)$ also

$$
G_{n}^{\hbar}(\Lambda, m)=<\psi_{\gamma_{n}, m}^{\hbar}, \hat{G}_{\gamma_{n}}(\Lambda) \psi_{\gamma_{n}, m}^{\hbar}>
$$

Then the limit (5.22) means that

$$
G(\Lambda, m)=\lim _{n \rightarrow \infty}\left[\lim _{\hbar \rightarrow \infty} G_{n}^{\hbar}(\Lambda, m)\right]
$$

where now the inner limit is taken at fixed $m, n$ and is meant in the sense of complex numbers. The limit (5.23) on the other hand means that

$$
G(\Lambda, m)=\lim _{\hbar \rightarrow \infty}\left[\lim _{n \rightarrow \infty} G_{n}^{\hbar}(\Lambda, m)\right]
$$

and will be much more difficult to check for a more general operator because the $\hbar$ corrections of the inner bracket might not converge. In our case, however, both limits are immediate and in fact reproduce $G(\Lambda)$ as we will show as an example in the first publication of 44.

To conclude, we have shown that there is an anomaly-free quantization of the Gauss constraint on the continuum Hilbert space $\mathcal{H}$ with the corrrect classical limit. The limit (5.22) says that the regularization procedure is meaningful while the limit (5.23) shows that the regulator can be removed without picking up divergencies and such that we obtain the correct classical limit.

\section{Non-Commutativity Issues}

The authors of [45] considered the following classical functions on $(M, \Omega)$

$$
E(S, f):=\int_{S}\left(* E_{i} f^{i}\right)(x)
$$

where $S$ is an oriented smooth (D-1)-dimensional submanifold of $\Sigma$ and $f^{i} \in \mathcal{S}$. Notice that $E(S, f)$ in contrast to our $P(S)$ of $(3.15)$ is not gauge covariant for any choice of $f$ and that $P(S) \neq E(S, f)$ since $P(S)$ depends explicitly on both $A$ and $E$ while (6.1) depends only on E.

In order to compute the Poisson brackets among the $E(S, f)$ induced by the symplectic structure $\Omega$ one should introduce, as in section 3.2.3, a one parameter family of surfaces $t \mapsto$ $S_{t}, t \in[-1,1], S_{0}=S$ and smooth regulator functions $g_{\epsilon}(t), \lim _{\epsilon \rightarrow 0} g_{\epsilon}(t)=\delta(t)$. One obtains regulated quantities

$$
E_{\epsilon}(S, f):=\int_{-1}^{1} d t g_{\epsilon}(t) \int_{X^{-1}\left(S_{t}\right)} d^{2} x\left(X^{*} f_{a}^{i} E_{i}^{a}\right)(x)
$$


at the aid of which we compute the Poisson brackets

$$
\left\{E(S, f), E\left(S^{\prime}, f^{\prime}\right)\right\}:=\lim _{\epsilon \rightarrow 0}\left\{E_{\epsilon}(S, f), E_{\epsilon}\left(S^{\prime}, f^{\prime}\right)\right\}_{\Omega}=0
$$

by $(3.13)$.

The authors of 45 now proceeded as follows :

Since, according to the symplectic structure of $\Omega$, we formally have $\left\{E_{i}^{a}(x), A_{b}^{j}(y)\right\}=\delta_{b}^{a} \delta_{i}^{j} \delta^{(D)}(x, y)$, they represented the operator $\hat{E}_{i}^{a}(x)$ by the functional derivative $i \delta / \delta A_{a}^{i}(x)$ defined on functions of smooth connections, substituted this derivative into (6.1), applied it to functions $f_{\gamma}$ of holonomies of smooth connections over a graph $\gamma$ and extended the final operator to distributional connections. The result is the following :

Without loss of generality we can subdivide the graph sufficiently and orient all the edges of $\gamma$ in such a way that any edge of $\gamma$ belongs to one of the following four categories : i) $e \cap S=\emptyset$, ii) $e \cap S=e$, iii) $e \cap S=e(0)$ and $e$ lies on the "up" side of $S$ or iv) $e \cap S=e(0)$ and $e$ lies on the "down" side of $S$. Notice that in case iii),iv) the edge is allowed to be tangent at $e(0)$. Denote the subset of edges belonging to category iii) and iv) respectively by $E_{S}^{u}(\gamma)$ and $E_{S}^{d}(\gamma)$ respecively and the subset of vertices in $S \cap\left(E_{S}^{u}(\gamma) \cup E_{S}^{d}(\gamma)\right)$ by $V_{S}(\gamma)$. Then

$$
\hat{E}(S, f) f_{\gamma}=i \hbar \sum_{p \in V_{S}(\gamma)} \frac{f^{i}(p)}{2}\left[\sum_{e(0)=p, e \in E_{S}^{u}(\gamma)} X_{i}^{e}-\sum_{e(0)=p, e \in E_{S}^{d}(\gamma)} X_{i}^{e}\right] f_{\gamma}
$$

where again $X_{i}^{e}$ denotes the right invariant vector field on the $e^{\prime}$ th copy of $G$. The expression (6.4) defines a self-consistent family of operators $\hat{E}_{\gamma}(S, f)$ defined on (a dense subset of) $\mathcal{H}_{\gamma}$.

The non-commutativity becomes now obvious by choosing for instance $S=S^{\prime}$ so that

$$
\left.\left[\hat{E}(S, f), \hat{E}\left(S, f^{\prime}\right)\right] f_{\gamma}=\hbar^{2} \sum_{p \in V_{S}(\gamma)} \frac{f^{i}(p) f^{j^{\prime}}(p) f_{i j}{ }^{k}}{2} \sum_{e(0)=p, e \in E_{S}^{u}(\gamma) \cup E_{S}^{d}(\gamma)} X_{k}^{e}\right] f_{\gamma}
$$

and even worse, one cannot write the right hand side as $\hat{E}\left(S,\left[f, f^{\prime}\right]\right)$ !

These problems can be overcome as follows:

Partition the surface $S$ into disjoint open pieces $S_{p}$ carrying the same orientation as $S$ such that $p$ is the only point of $V_{S}(\gamma)$ lying in $S_{p}$ and $\cup_{p \in V_{S}(\gamma)} S_{p}=S$ modulo boundary points. For each $e \in E_{S}^{u}(\gamma)$ or $e \in E_{S}^{d}(\gamma)$ respectively, deform $S_{p}$ in an arbitrarily small neighbourhood of $p$ into the direction of $e$ to a surface $S_{e}$ which intersects $e$ transversally in an interior point of $e$ but no other edge of $\gamma$ and which carries the same or opposite orientation as $S_{p}$. Obviously, these surfaces qualify as part of a dual decomposition of $\gamma$. We can now construct from these data the following function on $M$ which can also be considered as a function on $M_{\gamma}$

$$
E_{\gamma}(S, f):=\sum_{p \in V_{S}(\gamma)} f^{i}(p)\left[\sum_{e(0)=p, e \in E_{S}^{u}(\gamma)} P_{i}^{e}-\sum_{e(0)=p, e \in E_{S}^{d}(\gamma)} P_{i}^{e}\right]
$$

which obviously has classically nothing to do with $E(S, f)$. Nevertheless, the results of section 5 tell us that its quantization exactly agrees with (6.4), moreover, the algebra of operators of this kind reflects precisely the symplectic structure $\Omega_{\gamma}$ which is derived from $\Omega$.

In conclusion, we have demonstrated that the family of operators (6.4) can be considered as bona fide quantizations of a family of classical functions which do not Poisson commute with respect to $\Omega$ and therefore the apparent contradiction between classical Poisson bracket algebra and quantum commutator algebra pointed out in 45 evaporates.

The discussion of this section seems to reveal that not only there is ambiguity in quantizing a given classical functions due to the always existing possibility to add $\hbar$ corrections, but also vice versa that there is an ambiguity in taking the classical limit, in the sense that one 
and the same operator can be considered as a quantization of two different classical functions. However, this is not the case if we insist that we begin with a classical phase space and operators have to have a commutator algebra reflecting the classical Poisson bracket algebra. From this point of view, the functions (6.1) must not be considered as classical limit of the operators (6.4) ! One can still argue that the $S^{e}$ are quite arbitrary and that the classical limit is therefore not really well defined, but as already said before, a well-defined classical limit can only be expected in the limit of $\gamma \rightarrow \Sigma$ in a definite way in which the arbitrariness of the $S^{e}$ is lost.

Besides, the functions (6.4) are unphysical already from the point of view of the Gauss constraint : it is impossible to build from them gauge invariant observables except in the limit of infinitesimal faces where they have been used to build geometrical operators [16, 17, 18, 19, 20]. However, in that limit we get anyway $E(S, f) \rightarrow P\left(S^{e}\right)_{i} f^{i}(e(0))$ so that one can equally well construct these operators from the $P\left(S^{e}\right)_{i}$ and so there is finally complete agreement between all the results previously obtained in the literature and our approach.

\section{Acknowledgements}

We thank O. Winkler for a careful reading of the manuscript.

\section{A The Symplectic Structure for $G=S U(2), U(1)$ as a Two-Form}

Let us first fix our conventions :

For a $p$-form $\omega=\omega_{a_{1} . a_{p}} d x^{a_{1}} \otimes . . \otimes d x^{a_{p}}=\omega_{a_{1} . . a_{p}} d x^{a_{1}} \wedge . . \wedge d x^{a_{p}}$ on a finite dimensional manifold $M$ with $\omega_{a_{1} . a_{p}}=\omega_{\left[a_{1} . . a_{p}\right]}$ we define exterior differential, interior products with vector fields $v$ and Lie derivatives respectively by

$$
\begin{aligned}
d \omega & =\partial_{a} \omega_{a_{1} . . a_{p}} d x^{a} \wedge d x^{a_{1}} \wedge . \wedge \wedge d x^{a_{p}} \\
i_{v} \omega & =p v^{a} \omega_{a a_{1} . . a_{p-1}} d x^{a_{1}} \wedge . \wedge d x^{a_{p-1}} \\
\mathcal{L}_{v} \omega & =\left[i_{v} d+d i_{v}\right] \omega
\end{aligned}
$$

Let now $(M, \Omega)$ be a finite dimensional symplectic manifold and $f \in C^{\infty}(M)$. We define the Hamiltonian vector field $\chi_{f}$ of $f$ by

$$
i_{\chi_{f}} \Omega+d f=0
$$

and the Poisson bracket of $f, g \in C^{\infty}(M)$ with respect to $\Omega$ by

$$
\{f, g\}:=-i_{\chi_{f}} i_{\chi_{g}} \Omega=\chi_{f}(g)=i_{\chi_{f}} d g
$$

If $\Omega=d \Theta$ is exact then $\Theta$ is called a symplectic potential for $\Omega$. Here is a quick method of how to go backwards from $\{.,$.$\} to \Omega$ :

Introduce local coordinates $z^{\alpha}$ on $M$ and corresponding tensor components $\Omega=\frac{1}{2} \Omega_{\alpha \beta} d z^{\alpha} \wedge d z^{\beta}$ and define by $\Omega^{\alpha \gamma} \Omega_{\gamma \beta}=\delta_{\beta}^{\alpha}$ the inverse tensor. Then the Hamiltonian vecor field of any function $f$ is given by $\Omega^{\alpha \beta} \partial_{\beta} f$. Thus $\left\{z^{\alpha}, z^{\beta}\right\}=\Omega^{\gamma \delta}\left(\partial_{\delta} z^{\alpha}\right)\left(\partial_{\gamma} z^{\beta}\right)=-\Omega^{\alpha \beta}$ and so we just have to invert the matrix of Poisson brackets to obtain $\Omega$.

The reader may verify that with our conventions the symplectic potential $\Theta=p d q$ of $M=T^{*} \mathbb{R}$ leads to $\{p, q\}=1$.

\section{A.1 $U(1)$}

The Lie algebra of $U(1)$ is spanned by $i$ (imaginary unit) and is therefore Abelian. Let $h \in U(1)$ be a complex number of modulus one. We want to compute the symplectic structure $\Omega$ on $M=$ 
$T^{*} U(1)$ corresponding to the brackets $\{h, h\}=0=\{p, p\},\{p, h\}=\frac{i}{2} h$. Let $z^{1}=p, z^{2}=h$, then $\Omega^{\alpha \beta}=-i h \epsilon^{\alpha \beta} / 2$ where $\epsilon^{\alpha \beta}$ is the completely skew tensor density of weight one. Thus $\Omega_{\alpha \beta}=2 \epsilon_{\alpha \beta} /(i h)$ and $\Omega=2 d p \wedge d h /(i h)=-2 i d p \wedge d \ln (h)$ which equals $2 d p \wedge d \varphi$ locally if we write $g=\exp (i \varphi)$. $\Omega$ is real and exact with symplectic potential $\Theta=-2 i p d \ln (h)$.

\section{A.2 $S U(2)$}

This time there is considerably more work involved and we will only sketch the main steps.

Recall the following normalization conditions for our generators $\operatorname{tr}\left(\tau_{i} \tau_{j}\right)=-2 \delta_{i j},\left[\tau_{i}, \tau_{j}\right]=$ $2 \epsilon_{i j k} \tau_{k}$ (for instance $\tau_{j}=-i \sigma_{j}$ the later being the standard Pauli matrices). Let us introduce the following global group coordinates

$$
S:=\operatorname{tr}(h), T^{i}:=\operatorname{tr}\left(\tau_{i} h\right)
$$

then $h=\left(S-T^{i} \tau_{i}\right) / 2$ and we have the following relation $4-S^{2}=\left(T^{i}\right)^{2}$. Thus, instead of working with $S, T^{i}$ we can work with $\epsilon, T^{i}$ where $\epsilon=S /|S|=0, \pm 1$ is a discrete parameter. From (3.24) - (3.26) we compute the fundamental Poisson brackets

$$
\begin{aligned}
\left(\Omega^{-1}\right)^{j k} & :=\left\{T^{j}, T^{k}\right\}=\{\epsilon, \epsilon\}=\left\{T^{i}, \epsilon\right\}=0 \\
\left(\Omega^{-1}\right)_{j}{ }^{k} & :=\left\{p_{j}, T^{k}\right\}=-\frac{1}{2}\left[\epsilon \delta_{j k} \sqrt{4-\left(T^{m}\right)^{2}}-\epsilon_{j k l} T^{l}\right] \\
\left\{p_{j}, \epsilon\right\} & =0 \\
\left(\Omega^{-1}\right)_{j k} & :=\left\{p_{j}, p_{k}\right\}=-\epsilon_{j k l} p_{l}
\end{aligned}
$$

and certainly $\left(\Omega^{-1}\right)^{k}{ }_{j}=-\left(\Omega^{-1}\right)_{j}{ }^{k}$. Thus, our task is to invert the $6 \times 6$ matrix $\Omega^{-1}$ defined in (A.7). We do not worry about the discrete parameter $\epsilon$ which Poisson commutes with everything in what follows.

Let us introduce the $3 \times 3$ matrix $\Lambda(v)$ defined for every vector $v$ by $\Lambda(v)_{i j}:=\epsilon_{i j k} v^{k}$. Let also $z^{\alpha}=T^{\alpha}, \alpha=1,2,3 ; z^{\alpha}=p_{\alpha-3}, \alpha=4,5,6$ and $\Omega^{\alpha \beta}:=\left(\Omega^{-1}\right)^{\alpha \beta}=\left\{z^{\alpha}, z^{\beta}\right\}$. Then $\Omega^{-1}$ is explicitly given by

$$
\Omega^{-1}=\frac{1}{2}\left(\begin{array}{cc}
0 & -S 1_{3}+\Lambda(T) \\
S 1_{3}-\Lambda(T) & 2 \Lambda(p)
\end{array}\right)
$$

Thus, the $6 \times 6$ matrix decomposes into four blocks of $3 \times 3$ matrices. For the matrix $\Omega$ we now make a similar block matrix ansatz

$$
\Omega=2\left(\begin{array}{cc}
\Lambda(a) & B \\
-B^{T} & 2 \Lambda(c)
\end{array}\right)
$$

and study the relations that we obtain from $\Omega \Omega^{-1}=1_{6}$ for the vectors $a, c$ and the $3 \times 3$ matrix $B$. Using the relations $\Lambda(U) \Lambda(v)=v \otimes u-(u, v) 1_{3}$ one finds after very lengthy calculations the result

$$
\Omega=\frac{1}{2}\left(\begin{array}{cc}
2\left[\Lambda(p)+\frac{T \otimes p-p \otimes T}{S}\right] & S 1_{3}-\Lambda(T)+\frac{T \otimes T}{S} \\
-S 1_{3}-\Lambda(T)-\frac{T \otimes T}{S} & 0
\end{array}\right)
$$

The matrix (A.10) is singular at $S=0$ but we will see that this is merely a coordinate singularity by simply working out $\Omega=\frac{1}{2} \Omega_{\alpha \beta} d z^{\alpha} \wedge d z^{\beta}$. The result is

$$
\Omega=\frac{1}{2}\left[\left(\Lambda(p)+\frac{T \otimes p-p \otimes T}{S}\right)_{i j} d T^{i} \wedge d T^{j}+\left(S 1_{3}-\Lambda(T)+\frac{T \otimes T}{S}\right)_{i}{ }^{j} d T^{i} \wedge d p_{j}\right.
$$

and we find the following global symplectic potential (so $\Omega=d \Theta$ is exact)

$$
\Theta=\frac{1}{2} p_{j}\left(\epsilon_{i j k} T_{k} d T_{i}-S d T^{j}+T^{j} d S\right)
$$


from which regularity is obvious. We also find the following locally defined momentum conjugate to $T^{i}$

$$
\pi_{i}=\frac{1}{2}\left(\epsilon_{i j k} T_{k}-S \delta_{i j}+\frac{T^{i} T^{j}}{S}\right) p_{j}
$$

and indeed after lengthy calculations using (A.7) we find that $\left\{T^{i}, T^{j}\right\}=\left\{\pi_{i}, \pi_{j}\right\}=0,\left\{p_{i}, T^{j}\right\}=$ $\delta_{i}^{j}$. Clearly, $\pi_{i}, T^{i}$ can only be local Darboux coordinates otherwise we would have displayed $T^{*} S U(2) \equiv T^{*} S^{3}$ as $T^{*} B^{2}$ where $B^{2}$ is a solid ball in $\mathbb{R}^{3}$ (we are missing the discrete information coming from $\epsilon$ ).

The form (A.12) could be the starting point of symplectic reduction of $\left(M_{\gamma}, \Omega_{\gamma}\right)$ by the Gauss constraint $G_{\gamma}(\Lambda)$ at the classical level already using methods from geometric quantization which has not been done so far in the literature to the best of our knowledge. However, the manifold $M_{\gamma}$ reduced by $G_{\gamma}$ is rather singular except in the case of only one copy of $G$ and therefore is unattractive.

\section{References}

[1] A. Ashtekar, Phys. Rev. Lett. 57 (1986) 2244, Phys. Rev. D36 (1987) 1587

[2] A. Ashtekar, in "Mathematics and General Relativity", American Mathematical Society, Providence, Rhode Island, 1987

[3] F. Barbero, Phys. Rev. D51 (1995) 5507

F. Barbero, Phys. Rev. D51 (1995) 5498

[4] T. Thiemann, Physics Letters B 380 (1996) 257-264, gr-qc/960688

[5] R. Gambini, A. Trias, Phys. Rev. D22 (1980) 1380

C. Di Bartolo, F. Nori, R. Gambini, A. Trias, Lett. Nuov. Cim. 38 (1983) 497

R. Gambini, A. Trias, Nucl. Phys. B278 (1986) 436

[6] T. Jacobson, L. Smolin, Nucl. Phys. B299 (1988) 295

[7] A. Ashtekar and C.J. Isham, Class. Quantum Grav. 9 (1992) 1433

[8] A. Ashtekar, J. Lewandowski, "Representation theory of analytic holonomy $C^{\star}$ algebras", in "Knots and Quantum Gravity", J. Baez (ed), Oxford University Press, Oxford, 1994

A. Ashtekar, J. Lewandowski, Journ. Geo. Physics 17 (1995) 191

A. Ashtekar and J. Lewandowski, J. Math. Phys. 36 (1995) 2170

[9] D. Marolf and J. M. Mourão, "On the support of the Ashtekar-Lewandowski measure", Commun. Math. Phys. 170 (1995) 583-606

[10] A. Ashtekar, J. Lewandowski, D. Marolf, J.Mourão, T. Thiemann, Journ. Math. Phys. 36 (1995) 6456-6493, gr-qc/9504018

[11] C. Rovelli, L. Smolin, Nucl. Phys. B331 (1990) 80

[12] R. Gambini, J. Pullin, "Loops, Knots, Gauge Theories and Quantum Gravity", Cambridge University Press, Cambridge, 1996

[13] T. Thiemann, Journ. Math. Phys. 39 (1998) 1236-48, hep-th/9601105

[14] R. De Pietri, C. Rovelli, Phys. Rev. D54 (1996) 2664

R. De Pietri, Class. Quantum Grav. 14 (1997) 53

[15] T. Thiemann, Journ. Math. Phys. 39 (1998) 3372-92, gr-qc/9606092

[16] C. Rovelli, L. Smolin, Nucl. Phys. B 442 (1995) 593; Erratum : Nucl. Phys. B 456 (1995) 734

[17] A. Ashtekar, J. Lewandowski, Class. Quantum Grav. 14 A55-81 (1997)

[18] R. Loll, Nucl. Phys. B460 (1996) 143

R. Loll, Class. Quantum Grav. 14 (1997) 1725

R. Loll, Nucl. Phys. B500 (1997) 405

[19] A. Ashtekar, J. Lewandowski, Adv. Theo. Math. Phys. 1 (1997) 388-429

[20] T. Thiemann, Journ. Math. Phys. 39 (1998) 3347-71, gr-qc/9606091]

[21] T. Thiemann, Class. Quantum Grav. 15 (1998) 839-73, gr-qc/9606089 
[22] T. Thiemann, Class. Quantum Grav. 15 (1998) 875-905, gr-qc/9606090

[23] T. Thiemann, Class. Quantum Grav. 15 (1998) 1249-80, gr-qc/9705018

[24] T. Thiemann, Class. Quantum Grav. 15 (1998) 1281-1314, gr-qc/9705019

[25] T. Thiemann, Class. Quantum Grav. 15 (1998) 1487-1512, gr-qc/9705021

[26] T. Thiemann, Class. Quantum Grav. 15 (1998) 1463-85, gr-qc/9705020

[27] T. Thiemann, Class. Quantum Gravity 15 (1998) 1207-47, gr-qc/9705017

[28] T. Thiemann, "Quantum Spin Dynamics (QSD) : VIII. The Classical Limit", to appear

[29] M. Reisenberger, C. Rovelli, Phys. Rev. D56 (1997) 3490-3508

[30] J. Baez, Class. Quantum Grav. 15 (1998) 1827-58

[31] F. Markopoulou, L. Smolin, Phys. Rev. D58 (1998) 084032

[32] J. W. Barret, L. Crane, Class. Quantum Grav. 14 (1997) 2113

[33] L. Freidel, K. Krasnov, Adv. Theor. Math. Phys. 2 (1999) 1183

[34] K. Krasnov, Gen. Rel. Grav. 30 (1998) 53-68

[35] A. Ashtekar, C. Beetle, S. Fairhurst, Class. Quantum Grav. 17 (2000) 1317

[36] A. Ashtekar, A. Corichi, K. Krasnov, "Isolated horizons : The classical phase space", gr-qc/9905089

[37] A. Ashtekar, C. Beetle, S. Fairhurst, Class. Quantum Grav. 16 (1999) L1-L7, grqc/9812065

[38] A. Ashtekar, K. Krasnov, "Quantum Geometry and Black Holes", gr-qc/9804039

[39] A. Ashtekar, J. Baez, A. Corichi, K. Krasnov, Phys. Rev. Lett. 80 (1998) 904

[40] A. Ashtekar, "Interface of General Relativity, Quantum Physics amd Statistical Mechanics : Some Recent Developments", gr-qc/9910101

[41] S.A. Fulling, "Aspects of Quantum Field Theory in Curved Space-Time", Cambridge University Press, Cambridge, 1989

[42] T. Thiemann, "Gauge Field Theory Coherent States (GCS) : I. General Properties", hep-th/0005233

[43] T. Thiemann, O. Winkler, "Gauge Field Theory Coherent States (GCS) : II. Peakedness Properties", hep-th/0005237

T. Thiemann, O. Winkler, "Gauge Field Theory Coherent States (GCS) : III. Ehrenfest Theorems", hep-th/0005234

T. Thiemann, "Gauge Field Theory Coherent States (GCS) : V. Extension to Higgs Fields and Fermions", to appear

[44] T. Thiemann, O. Winkler, "Gauge Field Theory Coherent States (GCS) : IV. Infinite Tensor Product and Thermodynamic Limit", hep-th/0005235

H. Sahlmann, T. Thiemann, O. Winkler, "Gauge Field Theory Coherent States (GCS) : VI. Photons and Gravitons Propagating on Quantum Spacetimes", to appear H. Sahlmann, T. Thiemann, O. Winkler, "Gauge Field Theory Coherent States (GCS) : VII. The Non-Perturbative $\gamma$-Ray Burst Effect", to appear

[45] A. Ashtekar, A. Corichi, J.A. Zapata, Class. Quantum Grav. 15 (1998) 2955

[46] J. Baez, S. Sawin, "Functional Integration on Spaces of Connections", q-alg/9507023

J. Baez, S. Sawin, "Diffeomorphism Invariant Spin-Network States", q-alg/9708005

[47] J. Lewandowski, T. Thiemann, Class. Quantum Grav. 16 (1999) 2299-2322, grqc/9901015

[48] C. Rovelli, L. Smolin, "Spin networks and quantum gravity" pre-print CGPG-95/4-1, Phys. Rev. D52 (1995) 5743

[49] J. Baez, Adv. Math. 117 (1996) 253-272

[50] A. Rendall, Class. Quantum Grav. 10 (1993) 605

A. Rendall, "Adjointness Relations as a Criterion for Choosing an Inner Product", grqc/9403001

[51] E. Hebey, "Sobolev Spaces on Riemannian manifolds", Lecture Notes in Mathematics 1635, Springer Verlag, Berlin, 1996 
[52] Y. Choquet-Bruhat, C. DeWitt-Morette, "Analysis, Manifolds and Physics", part I, North Holland, Amsterdam, 1989

[53] S. Lang, "Differential Manifolds", Addison Wesley, Reading, Massachusetts, 1972

[54] H. Seifert, W. Threlfall, "Lehrbuch der Topologie", Chelsea Publishing Company, New York, 1980

[55] J. Kogut, L. Susskind, Phys. Rev. D 11 (1975) 395

P. Renteln, L. Smolin, Class. Quantum Grav. 6 (1989) 275

R. Loll, Nucl. Phys. Proc. Suppl. 57 (1997) 255

R. Loll, Class. Quantum Grav. 15 (1998) 799

R. Loll, gr-qc/9805049, http://www.livingreviews.org/.

[56] N. J. Vilenkin, "Special functions and the theory of group representations", American Mathematical Society, Povidence, Rhode Island, 1968

[57] Y. Yamasaki, "Measures on Infinite Dimensional Spaces", World Scientific, Singapore, 1985 